\title{
University of Ulm
}

Institute of Anatomy and Cell Biology

Prof. Dr. med. Tobias M. Böckers

\section{Developmental and Functional Nature of Human iPSC derived Motoneurons}

\author{
Dissertation \\ for obtaining the doctoral degree (Dr.med.) \\ of the Medical Faculty of the University UIm
}

submitted by

Marianne Stockmann

from Buchloe, Bayern

2012 
Incumbent Dean

$1^{\text {st }}$ Correspondent

$2^{\text {nd }}$ Correspondent

Day of Dissertation
: Prof. Dr. Thomas Wirth

: $\quad$ Prof. Dr. S. Liebau

: Prof. Dr. A. Ludolph

: 11.04.2014 
This thesis is dedicated to my parents 


\section{Table of Contents}

List of Abbreviations

1. Introduction 1

1.1. Amyotrophic lateral sclerosis and Model-systems $\quad 1$ in ALS research

1.2. Stem cells - Definition and Classes 3

1.3. Cell potency 5

1.4. Stem Cells of different species 9

1.5. Induced pluripotent Stem Cells 11

1.6. Differentiation of human Stem Cells 16

1.7. Differentiation of human Stem Cells 20 into functional Motoneurons

1.8. Maturation and synaptic Connections of spinal Motoneurons 23

1.9. Aims of the thesis 26

2. Material and Methods 28

2.1. Materials 28

2.2. Cell culture 37

2.3. Lentivirus Production 59

2.4. Protein Biochemistry 64

2.5. Quantitative real time PCR 66

2.6. Transmission Electron Microscopy 70

2.7. Electrophysiology 71

2.8. Data Analysis 72

$\begin{array}{lll}\text { 3. } & \text { Results } & 73\end{array}$

3.1. Generation of human iPSCs from Keratinocytes 73

3.2. Differentiation of mature Motoneurons 75

3.3. Motoneuron Maturation 79

3.4. Development of synaptic Structures in 83 hiPSCs derived Motoneuron Cultures 
3.5. $\quad$ Formation of mature Synapses in hiPSCs

derived Motoneuron Cultures

3.6. Generation of functional neuromuscular Junctions 91

3.7. Development of primary rodent Motoneurons

$\begin{array}{ll}\text { 4. Discussion } & 100\end{array}$

4.1. Generation of human Stem Cells without ethical concerns 100

4.2. Stem Cell Model for human Motoneuron Maturation 101

4.3. The Stem Cell Model and human Motoneuron 109 Degeneration

4.4. Read out Systems for human Motoneuron Degeneration

4.5. Conclusions

5. Summary

6. List of literature

7. Figures and Tables 


\section{List of Abbreviations}

ACh

acetylcholine

$\mathrm{AChR}$

acetylcholinereceptor

AFP

alpha-feto-protein

ALS

amyotrophic lateral sclerosis

ALK

activin receptor like kinase

AMPA

a-amino-3-hydroxy-5-methyl-4-isoxazolepropionic acid

ANG

angiogenin

AP

action potential

AP -5

amino-5-phosphonovaleric acid

AZ

active zone

$\alpha-\mathrm{MHC}$

alpha myosin heavy chain

a-BTX

alpha bungarotoxin

BDNF

brain-derived neurotrophic factor

BMPs

bone morphogenetic proteins

BSN

bassoon

CAZ

cytomatrix at the active zone

cAMP

cyclic adenosine monophosphate

cDNA

complementary DNA

ChAT

choline acetyltransferase

c-MYC

v-myc myelocytomatosis viral oncogene homolog

$\mathrm{CM}$

conditioned medium

CNS

central-nervous-system

CNQX

6-cyano-7-nitroquinoxaline-2,3-dione

$\mathrm{CP}$

crossing point

CVs

clear large vesicles

DAPI

4',6-Diamidin-2-phenylindol

DCVs

dense-core vesicles

DIV

day in vitro

DMEM

Dulbecco's Modified Eagle Medium 
DNA

DRG

EB

ECCs

EDTA

EGCs

EGF

EGTA

ERK $1 / 2$

ESCs

ESRB

$\mathrm{EtOH}$

FACS

fALS

FBS

FGF

FGFR

FUS/TLS

GDNF

GFAP

HAMCs

HBSS

HB9 / MNR2

HESCs

HiPSCs

HSCs

HM

HMBS

hMT deoxyribonucleic acid

dorsal root ganglion

embryoid body

embryonic carcinoma cells

ethylenediaminetetraacetic acid

embryonic germ cells

epidermal growth factor

ethylene glycol tetra acetic acid

erk-related tyrosine kinase

embryonic stem cells

estrogen receptor 2 (ER beta)

ethanol

flow cytometry analysis

familiar ALS

fetal bovine serum

fibroblast growth factor

fibroblast growth factor receptor

fused in sarcoma/translated in liposacroma protein

glia-derived neurotrophic factor

glial fibrillary acidic protein

human amniotic mesenchymal cells

Hank's buffered salt solution

motoneuron and pancreas homebox 1

human embryonic stem cells

human induced pluripotent stem cells

human stem cells

hormone mix

hydroxymethybilane synathase

human myotubes 


\begin{tabular}{|c|c|}
\hline H3K4 & histone H3 lysine 4 \\
\hline H3K27 & histone H3 lysine 27 \\
\hline ICM & inner cell mass \\
\hline IGF2 & insulin growth factor 2 \\
\hline IGF1 & insulin growth factor 1 \\
\hline ISL1 & islet 1 \\
\hline JAK & janus kinase \\
\hline KIF4 & kruppel-like factor 4 \\
\hline LEFTY $1 / 2$ & left-right determination factor \\
\hline LIF & leukemia inhibitory factor \\
\hline MAPK & mitogen-activated protein kinase \\
\hline MAP2 & microtubule-associated protein 2 \\
\hline MBP1 & myelin basic protein 1 \\
\hline MESCs & mouse embryonic stem cells \\
\hline MEF & mouse embryonic fibroblasts \\
\hline MG & matrigel \\
\hline MND & motoneuron disease \\
\hline M & molar \\
\hline mRNA & messenger ribonucleic acid \\
\hline NANOG & nanog homebox \\
\hline NEAA & non essential amino acids \\
\hline NE & neuroepithelial \\
\hline NES & nestin \\
\hline $\mathrm{NEFH}$ & neurofilament, heavy polypeptide \\
\hline NGN2 & neurogenin 2 \\
\hline NGLN1 & neuroligin 1 \\
\hline Noggin & inhibitor of TGFß signaling via BMP4 inhibition \\
\hline NMDA & N-methyl-D-aspartate \\
\hline
\end{tabular}


NMj

NP cells

NRG1

NRXN1

NSCs

NTC

OCT4

OLIG2

PAX6

PAX7

PBS

PcG

PCR

PCLO

PEI

PFA

PLO

PM

PMN

PMT

PrMN

PI3-K

PSD

PTVs

RA

REF

RNA

rpm

ROCK

RT neuromuscular junction

neural precursor cells

neuregulin

neurexin 1

neural stem cells

non-template control

POU class 5 homebox 1

oligodendrocyte transcription factor 2

paired box gene 6

paired box gene 7

Phosphate buffered Saline

polycomb group

polymerase chain reaction

piccolo

polyethylenimine

paraformaldehydt

poly-L-ornithin

postsynaptic membrane

motoneuron progenitors

primary myotubes

primary motoneurons

phosphoinositide-3-kinase

postsynaptic density

piccolo-bassoon transport vesicles

retinoid acid

rat embryonic fibroblasts

ribonucleic acid

rounds per minute

Rho-associated, coiled-coil containing protein kinase reverse transcriptase 
SALS

SAM

SB431542

$\mathrm{SC}$

SCID

SCV

SEM

s.e.m.

$\mathrm{SHH}$

SMI-32 / NEFH

SOD1

$\operatorname{SOX} 1 / 2$

STAT3

SYP

SYT1

S1P

TARP/TDP43

TEM

TGF $\beta$

$\mathrm{TH}$

TUBB 3

UV

VaChT sporadic ALS

sterile alpha motif (domain)

inhibitor of TGFß signaling

synaptic cleft

severe combined immunodeficiency

synaptic vesicle

scanning electron microscopy

standard error of the mean

sonic hedgehog

neurofilament, heavy polypeptide

superoxide dismutase 1

sex determining region $\mathrm{Y}$-box $1 / 2$

signal transducer and activator of transcription

synaptophysin

synaptostagmin 1

sphingosine 1-phosphate

TAR DNA binding protein

transmission electron microscopy

transforming growth factor, beta 1

tyrosine hydroxlyase

neuron specific class III $\beta$-tubulin

Ultra violet

vesicular acetylcholine transporter 
Introduction

\section{Introduction}

\subsection{Amyotrophic lateral sclerosis and Model-systems in ALS research}

Motoneuron diseases (MNDs) are taken together as a group of neuropathies that selectively affect motoneurons. They all inherit a progressive loss of motoneuron function and death of both the upper motoneuron, which connects the primary motor cortex and its pyramidal cells with the second motoneuron, and/or the lower motoneuron, located in the spinal cord. On segmental height the cell soma of second motoneuron is located connecting the spinal cord with the voluntary striated muscles of the trunk and limbs. Currently no efficacious cures or treatments for these diseases such as Amyotrophic lateral sclerosis (ALS) or Kennedy's Disease (KD) exist.

ALS is the most common adult onset disorder of motoneurons with an incidence of 1-3 per 100.000 year [172] and has been described as early as 1869 by JeanMarie Charcot depicting the clinical phenotype of ALS-patients for the first time [84]. ALS is characterized by degeneration of both the lower motoneurons in the spinal cord and brain stem as well as of the upper motoneurons in the motor cortex [21, 172]. ALS patients typically suffer from progressive muscle weakness and wasting, pathologically brisk reflexes, sometimes involving the limb and bulbar muscles [172]. Up to now, two major forms of ALS can be distinguished: sporadic ALS (sALS), which makes up 90-95\% of the cases and familial ALS (fALS) responsible for $5-10 \%$ of the cases [139].

Current understanding of ALS pathogenesis suggests a complex interplay of various factors including oxidative stress, protein aggregation, mitochondrial dysfunction, excitotoxicity and impaired axonal transport [172]. Furthermore, a complex genetic background is strongly associated with ALS pathogenesis. The investigation of disease causing mutations of familial ALS initiated the enlightening of the subjacent cellular mechanism of ALS pathogenesis. In the past decades, several candidate genes, which cause or at least influence ALS pathogenesis have been found. At first, mutations in the superoxide dismutase 1 gene (SOD1) [160] have been reported to be responsible for 20-25\% of all familiar cases and for some sporadic cases [5, 85]. More recent discoveries concentrate on sequence changes in genes responsible for autosomal dominantly inherited forms of ALS. 
For example, disease causing mutations were found in the TAR DNA bindingprotein (TARDP/TDP-43) [141, 183], angiogenin [70], the fused in sarcoma/translated in liposacroma protein (FUS/TLS) [99, 203], as well as a missense mutation in the 150 subunit of Dynactin [153].

The identification of genes underlying the cellular mechanism in ALS pathogenesis provided the possibility of the development of different animal models for that disease - for example the SOD $1^{\text {G93A }}$ mouse [72]. Next to ALS mouse models, drosophila [28] and zebrafish [100, 210] have successfully been established as model systems to investigate ALS pathogenesis. The analyses of animal models are considered as a crucial step in ALS research and provide a more detailed understanding of the cellular and molecular mechanisms that may be involved in familial ALS. Although, the relevance of these findings in translation to the human system, specifically human motoneurons has to be evaluated critically. Currently there is no possibility to isolate viable human motoneurons from patients or from post mortem samples, verifying the findings from animal models of ALS.

In vitro systems of primary rodent motoneurons are another popular model system. Initially, cultures of rodent neurons have been used to analyze synaptogenesis [67] and neurogenesis [34]. Furthermore, the structure of neuromuscular junctions (NMjs) and its vulnerability in MNDs has been investigated [139]. Conventional rodent motoneuron transfections as well as lentiviral transductions of various constructs carrying ALS-causing mutations provide the possibility to study in vitro morphology and characteristics of different mutations [186]. These studies have led to the identification of intrinsic pathogenic processes of affected motoneurons including protein aggregation, cytoskeletal abnormalities, proteasome dysfunction and increased signs of apoptosis [14, 22]. Moreover neuromuscular synapses seem to be targeted in different forms of MNDs like ALS or SMA (spinal muscular atrophy) [139].

The establishment of human embryonic stem cells as well as the discovery of human induced pluripotent stem cells, followed by the successful differentiation into spinal motoneurons, provides a new model system which reflects human ALS pathogenesis in a more superior way. In the past decades directed differentiation of embryonic stem cells from mouse (mESCs) and human ( $\mathrm{hESCs}$ ) origin into several neural lineages including neuronal precursor cells, midbrain dopamine neurons as well as glutamatergic and gabaergic neurons has successfully been 
shown $[51,228,230]$. Various protocols have been developed to differentiate hESCs as well as induced human pluripotent stem cells (hiPSCs) into spinal motoneurons [40, 82, 212]. Moreover, they are deployed to identify pathogenetic aspects of several diseases and might additionally help to develop novel strategies for the cure of neurodegenerative disorders like ALS, cardiac muscle degeneration or diabetes [196].

The basis of developing and utilization of hiPSCs from somatic donor cells is an appropriate knowledge of the different stem cell classes and stages of cell potency.

\subsection{Stem Cells - Definition and Classes}

Stem cells are defined by their ability of self-renewal as well as to differentiate into different cell types of the organism and tissues. Totipotency is preserved by the zygote up to the eight-cell state of the morula and is defined as the ability of a cell to give rise to unlike cells. One single totipotent cell is able to develop a new organism. The Morula stage is followed by the development of the blastocyst containing two primary cell types: the "inner cell mass" (ICM) and the trophoblast. The trophoblast cells form a layer of cells surrounding the inner cells and the blastocyst cavity whereas the ICM also known as "embryoblast" gives rise to all later structures of the organism. This ability of the ICM is defined as pluripotency. In the past three different forms of pluripotent stem cells have been described.

\subsubsection{Pluripotent Stem Cells}

\subsubsection{Embryonic Stem Cells (ESCs)}

Embryonic stem cells (ESCs) are pluripotent stem cells derived from the inner cell mass of preimplantation embryos [195]. They provide the capacity of self-renewal while maintaining their potential to differentiate into different somatic cell types of all three germ layers [2, 157, 195, 197]. In 1981, ESCs were independently first derived from mouse embryos [54]. Around ten years later, the first stable human embryonic stem cell (hESCs) line derived from the ICM of a blastocyst was published by James Thomson [195]. Ongoing differentiation of the very early morula leads to the formation of a blastocyst containing outer trophoblast cells and 
undifferentiated, pluripotent inner cell mass. The ICM is able to differentiate into all three germ layers and primordial germ cells (PGC), the founder cells of male and female gametes [195, 214]. To date, most of the hESC lines were derived from day 5 to day 8 blastocystes generated for clinical intentions after in vitro fertilization [187]. Differences between hESCs and mouse ESCs (mESCs) are described in 1.2.6.

\subsubsection{Embryonic Germ Cells (EGCs)}

EGCs are derived from the developing gonadal ridge $[29,170]$ of mammalian fetuses. The derivation of human pluripotent stem cells from cultured human primordial germ cells was published by Shamblott and co-workers in 1998 [169].

\subsubsection{Embryonic Carcinoma Cells (ECCs)}

EGCs were formed within testicular teratocarcinomas which themselves develop from primordial germ cells $[96,184]$. Contrary to ESCs and EGCs, ECCs does not play a role in normal embryogenesis. ECCs can differentiate into all cell types representative of the three primary germ layers [149]. However, they are always aneuploid and usually show a reduced differentiation potential [133].

\subsubsection{Multipotent Stem Cells}

Multipotent stem cells are tissue specific stem cells, which retain the ability of selfrenewal and are able to differentiate to cells from multiple, but limited number of lineages [98, 188]. Adult multipotent stem cells remain during the whole life and are localized to the so-called "stem cell niches". Multipotent adult stem cells are responsible for the regeneration of different types of cells and tissues. Some examples are the epidermis of the skin or the intestinal epithelium. Multipotent stem cells include for example hematopoietic stem cells (HSCs), mesenchymal stem cells (MSCs) and neural stem cells (NSCs). However, in recent experiments it was shown that HSCs could be differentiated into neuron-like cells - a process called transdifferentiation [175].

NSCs have been described for the first time in 1992 by Reynolds and Weiss [159]. They can be directly isolated from brain $[73,107,189]$ and provide the possibility of differentiating into various types of neurons and glial cells [107]. 
Introduction

\subsubsection{Induced pluripotent Stem Cells (iPSCs)}

IPSCs are a type of pluripotent stem cells derived from an adult somatic cell. Reprogramming to pluripotency is usually achieved by inducing a "forced" expression of certain stem cell-associated genes including OCT4 and SOX2. IPSCs are similar to ESCs in many aspects such as expression of stem cells markers, self-renewal, chromatin methylation and differentiation capacity [192, 197].

\subsection{Cell Potency}

\subsubsection{Classes of Cell Potency}

The potency of a cell is defined by its differentiation potential.

\subsubsection{Totipotency}

Totipotency describes the ability of a single cell to divide and differentiate into all the different cells in an organism, including extraembryonic tissues. Human development begins with a fertilized egg and the generation of a single totipotent cell (zygote). In the first hours after fertilization, the zygote divides into identical totipotent cells which can differentiate into any of the three germ layers. After reaching the 16-cell state of the morula, the totipotent cells differentiate into cells that will form either the blastocyst's inner cells mass or the trophoblast. The ability of the ICM to give rise to all later tissues of the organism is defined as pluripotency.

\subsubsection{Pluripotency}

Pluripotency refers to a stem cell, which is able to differentiate into any of the three germ layers [195, 197]: the endoderm (e.g. the gastrointestinal tract or the lungs), the mesoderm (muscle, bone and blood) and the ectoderm (epidermal tissues and the nervous system). Although pluripotent cells are able to give rise to any fetal or adult cell type, they fail in developing an adult organism alone because they cannot form extraembryonic tissue such as the placenta.

\subsection{Induced Pluripotency}


Induced pluripotent stem cells are derived from non-pluripotent somatic cells.

\subsubsection{Multipotency}

Multipotency describes the ability of cells to differentiate into a limited number of lineage-specific cells or into closely related family of cells.

\subsubsection{Oligopotency}

Oligopotency refers to the capacity of progenitor cells to give rise to a few cells types. Typical oligopotent cells are the lymphoid or myeloid stem cells. Another example of progenitor cells are vascular stem cells which can differentiate into endothelial or smooth muscle cells.

\subsubsection{Monopotency}

Monopotent cells are highly differentiated and specialized cells which can differentiate into only cell type.

\subsubsection{Pluripotency of undifferentiated hESCs lines}

Regulation and maintenance of pluripotency is different between hESCs and mESCs. The current study was performed in the human system, thus pluripotency of hECSs is explained in detail whereas the differences of hESCs and mESCs are described in 1.4.1. HESCs express typical nuclear factors of the undifferentiated state such as OCT4 (POU5F1, POU class 5 homebox 1), SOX2 (SRY (sex determining region Y)-box 2) as well as NANOG. They also express alkaline phosphate and show high levels of telomerase activity [157, 187]. Furthermore they express additional pluripotent markers such as keratin antigens TRA-1-60 (PODXL, podocalyxin-like) and TRA-1-81 and the glycolipid antigen SSEA4 (Stage Specific Embryonic Antigen) [64]. The in vivo potential of differentiation of hESC lines is usually assessed by teratoma formation in SCID mice, tumors containing tissues originating from all three germ layers [142]. For in vitro differentiation human stem cells are usually cultured in suspension where they form threedimensional aggregates called embryoid bodies (EBs) mimicking the early stages of embryonic development [29, 64, 155].

HESCs are usually maintained in an undifferentiated state by culturing on feeder cells layers, mostly mouse embryonic fibroblasts (MEFs) [42, 168, 214] or rat 
embryonic fibroblasts (REFs) [108]. Alternatively, hESCs can be cultured permanently on feeder cells from human tissues like human foreskin fibroblasts [127, 147], human bone marrow stromal cells or human amniotic mesenchymal cells (hAMCs) [227]. Feeder cells support the survival and the preservation of an undifferentiated state via supply of some critical factors either to promote selfrenewal or to suppress differentiation [214, 217]. Among these growth factors are TGFB (transforming growth factor beta), Inhba (inhibin beta A) and Grem1 (gremlin 1) have been shown to be secreted from MEFs and REFs and facilitate hESCs self-renewal [108].

Moreover, hESCs can be cultured under feeder free conditions on extracellular proteins like Matrigel (a complex mixture of extracellular matrix from EngelbrethHolm-Swarm tumor) or Laminin coating using e.g. MEF-conditioned media [78, 214], mTeSR1-Medium [78, 119] or StemPro Media [119]. Lately James Thomson and colleagues re-optimized the basic components of hESCs and hiPSCs culture media in the absence of albumin and B-mercaptoethanol, and developed a completely defined medium which is called E8 (eight components, including DMEM/F12) [26].

\subsubsection{Extrinsic Regulation of Pluripotency in hESCs}

In recent years, extrinsic factors, which promote self-renewal and suppress differentiation, have been identified in order to propagate and differentiate pure populations of stem cells (Fig. 1).

FGF2 (fibroblast growth factor 2) was the first factor found to be crucial for the maintenance of pluripotency in hESCs $[2,150]$. HESCs express three out of the four FGF-receptors (FGFR-1, FGFR-3 and -4) and produce FGF2 [49]. FGF2 activates ERK1 (extracellular signal-regulated kinase 1) and ERK2 promoting hESCs self-renewal and preventing differentiation [150]. Consequently, inhibition of these signals leads to stem cell differentiation [90, 103]. Recent studies report that mitogen activated protein kinase (MAPK) and phosphoinositide 3-kinase (PI3K) pathways are activated after FGF2-stimulation [42] and contribute to hESCs pluripotency. In addition, insulin-like growth factor 2 (IGF2) mediated signaling through PI3-K prevents hESCs from differentiating into endodermal lineage cells [150]. 
By affecting NANOG, which is crucial for ES cell self renewal, members of the transforming growth factor- $\beta$ family - such as TGF $\beta$ (transforming growth factor, beta 1), activin, nodal and bone morphogenetic proteins (BMPs) influence the maintenance of the undifferentiated stem cell state [150]. It has been shown that activin and nodal preserve stem cell pluripotency by activating SMAD2/3 signaling which leads to increased expression of LEFTY1/2 (left-right determination factor) which promotes self-renewal and suppress differentiation [86, 202]. One study detected that applying activin to hESCs leads to an increased production of FGF2 [216]. BMP4 has been shown to induce differentiation of hESCs to trophoectoderm [218, 219]. Thereby, the transcription factors SMAD1, SMAD5 and SMAD8, activated by BMP4, inhibit NANOG expression. The BMP4 inhibitor Noggin acts synergistically with FGF2 suppressing the BMP4-pathway, supposedly by a reduction of SMAD1 phosphorylation [219]. Inhibition of BMP4 signaling sustains the undifferentiated state of humane ES cells. 


\section{Introduction}

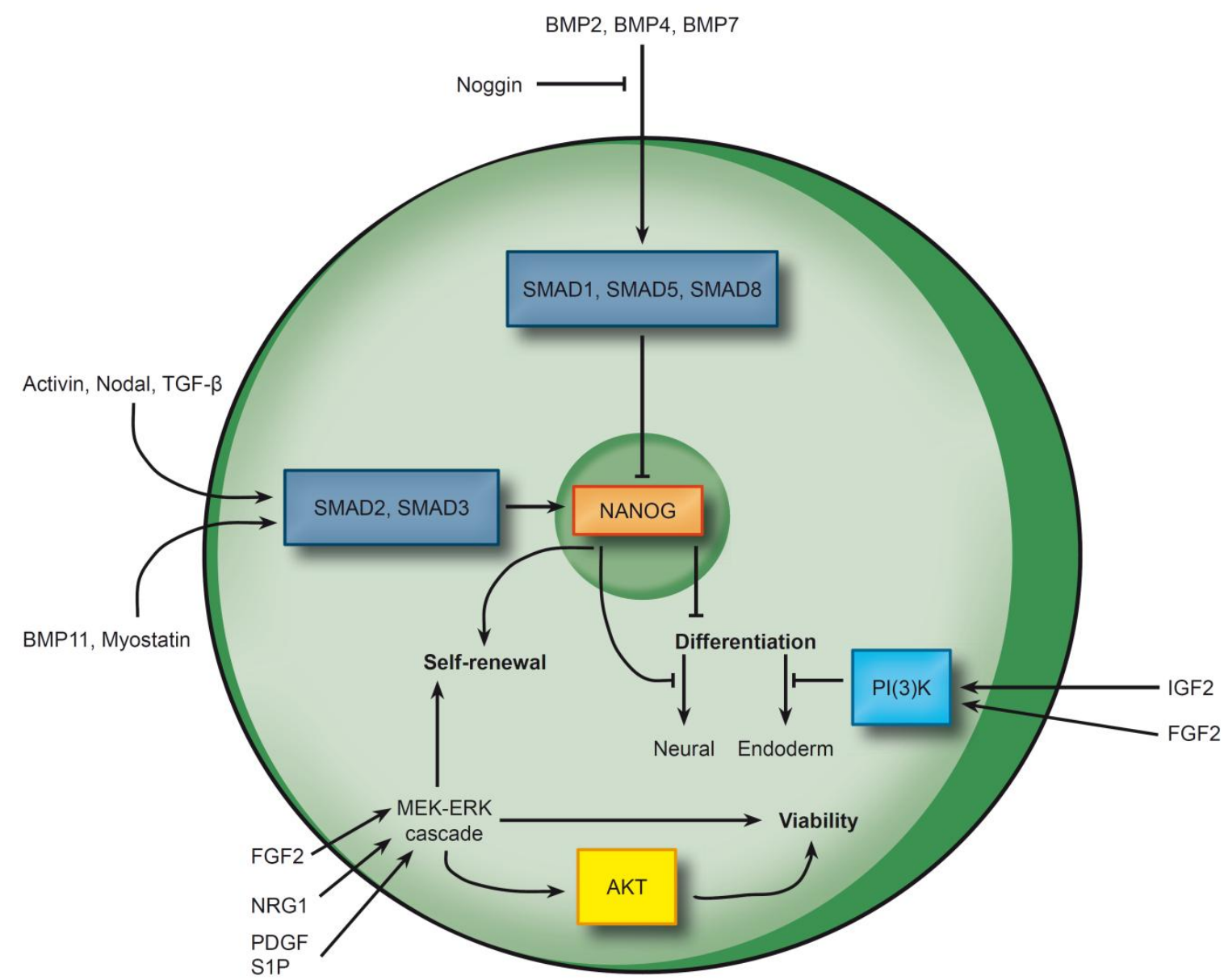

modified after Martin F. Pera \& Patrick P.L.Tam

\section{Figure 1 Extrinsic Regulation of Pluripotency}

Members of the TGFB-family including TGFB, activin and nodal, growth factors (GDFs, such as myostatin) and BMPs are involved in maintaining pluripotency and self-renewal of hESCs via NANOG signaling. FGF2, PDGF, S1P and NRG1 activate MEK-ERK receptor tyrosine kinase cascade allowing self-renewal und pluripotency. Furthermore activation of MEKERK cascade plays a crucial role in supporting hESCs viability (through inhibiting apoptosis and anoikis). In addition IGF2 as well as FGF2 mediate PI3-K signaling and inhibit differentiation into the endodermal lineage and support an undifferentiated state.

Abbreviations: BMPs = bone morphogenic proteins, FGF2 = fibroblast growth factor 2, IGF2 = insulin growth factor 2, NRG1 $=$ neuregulin, $\mathrm{PDGF}=$ platelet-derived growth factor, $\mathrm{PI} 3 \mathrm{~K}=$ phosphoinositide-3-kinase, $\mathrm{S} 1 \mathrm{P}=$ sphingosine 1-phosphate, TGFB = transforming growth factor $B$

\subsection{Stem Cells of different species}

\subsubsection{Human embryonic Stem Cells versus Mouse embryonic Stem Cells}

That there are obvious differences between mouse and human cells becomes visible already from colony morphology. HESCs show a flattened morphology, whereas mESCs grow as packed colonies [76]. 
Furthermore, most female mESCs maintain both $X$ chromosomes in an active state while the $X$ chromosome remains inactivated in human female ES cells [76, 176]. HESCs and mESCs express different transcription factors and surface markers. For example SSEA1, a cell-surface marker characteristic in mESCs, is absent in hESCs. Other surface markers like SSEA3, SSEA4, TRA-1-60 and TRA1-81 are expressed by hESCs and are missing in mESCs [224]. In mESCs BMP acts as an inhibitor of differentiation via the SMAD pathway [214] whereas BMP4 induces differentiation into trophoblast cells in human ES cell culture [214]. In addition, pathways controlling pluripotency and differentiation also differ between mouse and human stem cells. For maintenance of pluripotency, mESCs depend on leukemia inhibitory factor (LIF) [177, 222, 224, 231]. Binding of LIF to the gp130 receptor induces dimerization of LIFR/gp130 receptors and activates Janusassociated tyrosine kinases (JAK). Activated JAK leads to an activation of STAT3 (signal transducer and activator of transcription 3) and MAPK (mitogen activated protein kinase) cascades, maintaining an undifferentiated state in mouse stem cells. LIF and its related cytokines fail in supporting pluripotency in hESCs. In hESC culture FGF2 mediated signaling plays a crucial role in promoting selfrenewal and maintaining an undifferentiated state $[2,150]$. Recently, Jaenisch and co-workers could show that the pluripotent state of hESCs corresponds to that of mouse-derived epiblast stem cells (mEpiSCs) indicating that mESCs are in a more naïve pluripotent state than hESCs and mEpiSCs (primed pluripotent state) [76]. Subsequently, Jaenisch and co-worker converted the identity of conventional hESCs into a more immature state similar to the state of mESCs [76]. Ectopic induction of OCT4, KIF4 and KIF2 factors combined with LIF and the inhibition of GSK3 (glycogen synthase kinase 3) activity and mitogen-activated protein kinase (ERK 1/2) pathway results in an immature state of hESCs. In contrast to conventional hESCs these converted hESCs show an X-chromosome activation state $(\mathrm{XaXa})$, a gene expression file and signaling pathway properties very similar to mESCs [76].

\subsubsection{Mouse embryonic Stem Cells versus Rat embryonic Stem Cells}

Rat embryonic stem cells (rESCs) were derived by research teams led by Austin Smith in Cambridge and Qi-Long Ying of the University of Southern California [23, 104]. Although rat and mouse embryogenesis is very similar during the early 
stages of development, the early embryos differ significantly in their differentiation capacity in vitro or in vivo when they are transplanted to an ectopic site [104]. Several groups have tried to derive and culture rESCs under the same condition as mESCs, however no authentic rECS line could be established [104, 201]. The biggest problem has been the lack of appropriate culture conditions to sustain selfrenewal.

The key to success was finding a combination of small molecules (3i medium) able to prevent differentiation which was described by Smith and Ying in mESCs [23, 104]. Ying used three molecules: CHIR99021 (a GSK3 inhibitor), PD184352 (a MEK inhibitor), and SU5402 (an FGF receptor inhibitor), whereas Smith used only two inhibitors and left out the SU5402. These inhibitors switch off different ways of differentiation. In mESCs cultures, cultured under 3 i conditions, LIF was not longer necessary [23, 104].

The next step was to culture rESCs under $3 \mathrm{i}$ conditions and finally competent rESCs could be derived from rat blastocysts [104]. To note, inhibition of the GSK3 activity and the MEK pathway was also used by Jaenisch and co-workers to convert conventional hESCs into a more immature state (similar to mESCs) [76].

RESCs have a similar expression pattern of cell surface markers with mESCs but not hESCs. Similar to mESCs, rESCs express SSEA-1 but not SSEA-4 [104]. Moreover, OCT4 and NANOG promoters have found to be unmethylated in rESCs [104].

RESCs are able to differentiate into all three germ layers in vitro, but formation of EBs was at much lower efficiency compared with mESCs [104]. RESCs derived in 3i medium are capable of forming germline chimeras [104].

Robust, well characterized ESC lines exist for human and mice. The next essential step in stem cells research was the invention of induced pluripotent stem cells where an adult somatic cell reattains pluripotency.

\subsection{Human induced pluripotent Stem Cells}

Since the first discovery and invention of the induced pluripotent stem cells (iPSCs) technology by Takashaki and Yamanaka [192] it is now possible to analyze and study cell development and differentiation on the basis of a gene 
defects in patient specific settings $[19,206]$. Both ESCs and iPSCs are pluripotent and able to differentiate into three primary germ layers [112] (Fig. 2). Accordingly, hiPSCs afford the great promise to generate human stem cells without embryo tissue or oocytes.

\subsubsection{Inducing Pluripotency}

Induced pluripotent stem cells are generated by reprogramming a differentiated (but mitotically active) somatic cell into pluripotent stem cell [148]. Over the years a number of techniques have been developed to induce pluripotency in human as well as in mouse somatic cells $[113,125,145]$.

\subsubsection{Nuclear Transfer}

In 2000 Munsie et al reported on the generation of pluripotent murine stem cells from reprogrammed adult somatic nuclei via nuclear transfer [138]. Subsequently, nuclear transfer has been well established with mouse models [7, 24]. The nucleus of a somatic donor cells is isolated and transferred into an unfertilized, enucleated egg [148]. After being inserted in the egg, the development of the embryo is stimulated via electrical pulse or chemical compounds increasing the intracellular free calcium concentration. As a consequence, the inserted somatic cell nucleus is reprogrammed by the host cell. After forming a blastocyst, the inner cell mass can be isolated to generate human embryonic stem cells [18, 205]. Various somatic cells including mammary epithelial cells, cumulus cells, oviductal cells, leukocytes, hepatocytes, neuronal cells, myocytes, lymphocytes, and germ cells have been successfully used as donor cells for the production of cloned animals via nuclear transfer [148].

\subsubsection{Cellular Fusion}

Fusion between different cell types has been used to study plasticity of the differentiated state [10, 79]. Usually, the phenotype of the less-differentiated cell type is more dominant [79]. According to this, another method of generating reprogrammed somatic cells is based on cell-fusion of pluripotent embryonic stem cells and somatic cells [132], [191]. Next to murine ES cells and murine embryonic germ cells [191, 190], human embryonic stem cells are used as an alternative to oocytes for reprogramming human somatic nuclei [33]. HESCs are fused with 
human fibroblasts and other human somatic cells forming hybrid cells exhibiting properties similar to pluripotent stem cells. This includes the de-methylation and reactivation of genes essential for pluripotency and the ability of differentiating into all three primary germ layers in vivo supporting the hypothesis that somatic chromosomes had undergone genetic reprogramming [79]. However, testing reprogramming at a functional level there is no convincing evidence that somatic donor nuclei had been completely reprogrammed and that hybrid cells obtain the ability of sustaining pluripotency or self-renewal and are able to differentiate in the absence of the ES-cell genome [79].

\subsubsection{Direct Reprogramming}

Recently, somatic cells of murine and human origin such as fibroblasts [192], keratinocytes [1, 108], NSCs [92] or CD34+ mobilized human peripheral blood cells [117] have been reprogrammed by addition of selective transcription factors including OCT4, SOX2 in combination with both C-MYC and KIF4, KIF4 alone [140], LIN28 and NANOG [223] or ESRB [55] (Fig. 2). It has been shown that OCT4, SOX2 and KIF4 work together in combination to maintain a pluripotent state $[92,116]$. Expression of these factors together with c-MYC may result in a series of epigenetic events influencing chromatin modifications and changes in DNA methylation leading to a pluripotent like cell [148].

Several techniques have been developed in order to introduce the reprogramming factors into the somatic nucleus including the use of lenti - or retroviruses and non-viral methods. However, the generation of iPS cells is limited to the low reprogramming efficiency [192] and the long duration of the reprogramming process $[75,101]$ compared to cell fusion or nuclear transfer. Aasen and coworkers could show that the reprogramming efficiency of human keratinocytes is at least 100-fold more efficient and two-fold faster compared with the reprogramming of human fibroblasts [1]. Moreover, reprogramming efficiency has been improved by chromatin modulation [75], application of chemical compounds [126], the use of synthetic modified mRNA [207] and using rat embryonic fibroblasts (REFs) instead of mouse embryonic fibroblasts (MEFs) [108]. HiPSCs generated from fibroblasts or keratinocytes are indistinguishable from hESCs in colony morphology, growth properties, expression of pluripotency associated surface and transcription markers, global gene expression profiles and 
differentiation potential in vivo and in vitro (detailed description in 1.2.5) [1, 80, 102, 192, 194].

Most human iPS cells are generated by viral transduction including (retrovirus and lentivirus) which integrates the reprogramming factors into the host genome. Contrary to the retrovirus, a lentivirus is able to infect both proliferating and nonproliferating cells. However, lentivirus particles are derived from immunodeficient viruses which display safety concerns. The viral transcripts is efficiently silenced in hiPSCs [1, 108, 211]. Mostoslavsky and co-workers developed a single polycistronic lentiviral "stem cell cassette" vector containing all four reprogramming factors OCT4, SOX2, c-MYC and KIF4 resulting in a higher reprogramming efficiency. The engineered polycistronic lentiviral backbone was further engineered by Sommer et al [179] and contains an IRES element separating two fusion cistrons. The two cistrons consist of OCT4 and SOX2 coding sequences fused to KIF4 and c-MYC, respectively, through the use of intervening sequences encoding self-cleaving 2A peptides (F2A and E2A) [178, 179].

The "stem cell cassette" is flanked by two lox-P sites provide and provides the possibility of generating iPSCs free from exogenous transgenes by excising the "stem cell cassette" via recombinant CRE-protein [178, 180]. 
Introduction

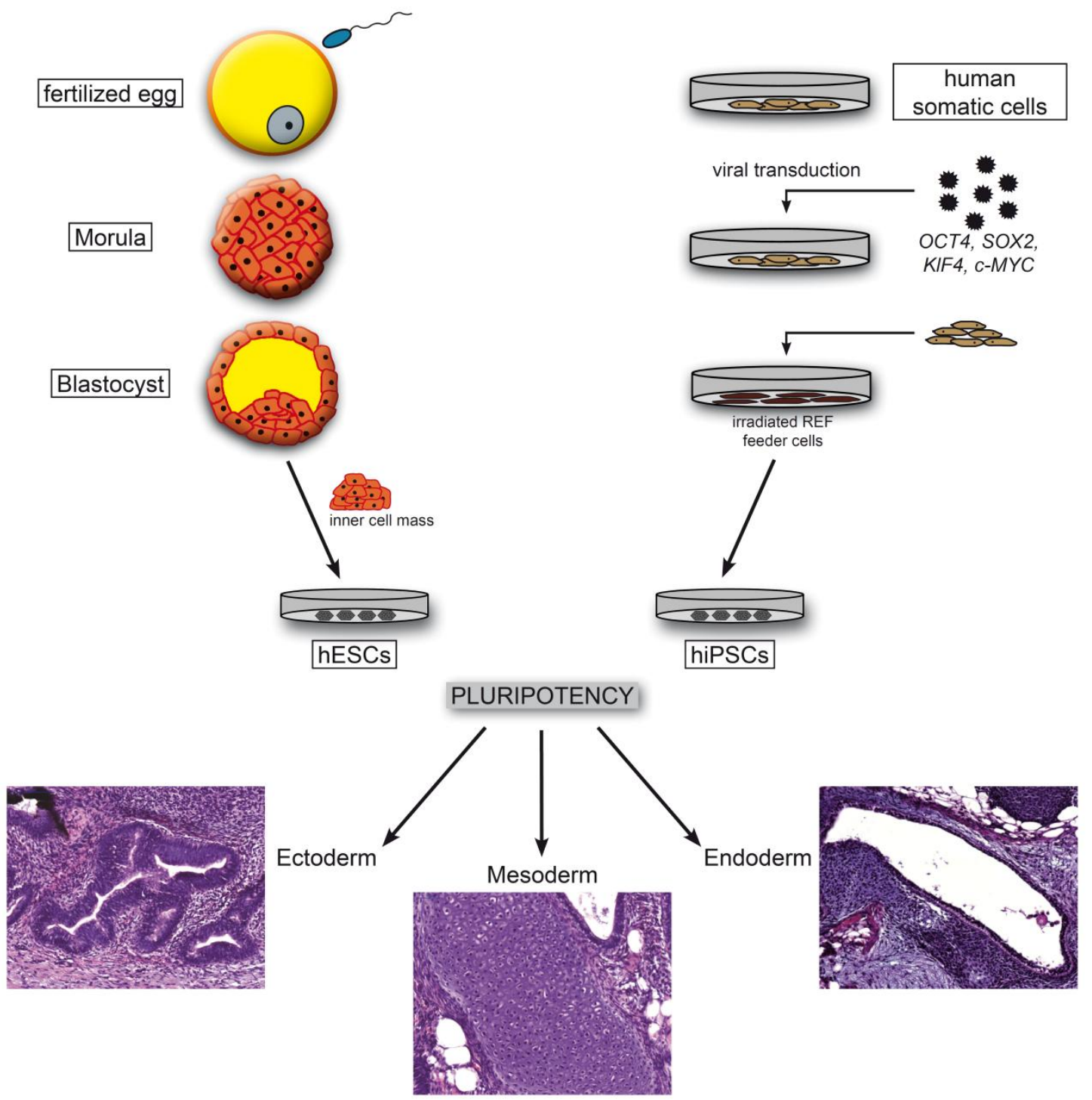

Figure 2 Stem Cell Development

Scheme of pluripotent stem cells' development in mammalian embryogenesis compared to the generation of induced pluripotent stem cells derived from human somatic cells.

Abbreviations: hESCs = human embryonic stem cells, hiPSCs = human induced pluripotent stem cells

\subsubsection{Human induced pluripotent Stem Cells vs. human embryonic Stem Cells}

\subsubsection{Morphology}

HiPSCs derived from human somatic cells usually exhibit an ES-like morphology including a round shape, a large nucleus versus cytoplasmic ratio and prominent nucleoli [1, 192]. Furthermore, hiPSCs as well as hESCs are evenly shaped with no space between. Colonies should have a clear border without signs of differentiation such as flattened cells or neural rosettes. 


\subsubsection{Pluripotency}

HiPSCs express genes and surface markers characteristic of hESCs including NANOG, OCT4, SOX2, as well as SSEA4, TRA-1-60 and TRA-1-80 [1, 108, 117, 212]. They express high levels of telomerase activity and are positive for alkaline phosphatase.

Additionally, global gene expression of hiPSCs clusters close to hESCs [55, 108, $117,211]$. Investigation of the DNA methylation level of OCT4 and NANOG promoters show that both loci are demethylated in hiPSCs [108, 211].

Recently, downstream targets genes of OCT4, NANOG and SOX2 have been identified. These genes contain many essential developmental regulators, which are also repressed by Polycomb Group complexes (PcG; a family of proteins which can remodel chromatin and play an essential role in regulating a number of key processes including lineage commitment). The chromatin of a number of these non-expressed target genes present a bivalent conformation in ESCs carrying both the "active" histone $\mathrm{H} 3$ lysine 4 (H3K4) methylation mark and the "repressive" histone H3 lysine 27 (H3K27) methylation mark [154]. Quantification of H3K4 and H3K27 methylation of hiPSCs shows a comparable enrichment for both histone modifications similar to normal hESCs [211].

Furthermore, female hiPSCs, similar to hESCs, show an inactivation of the somatically silenced $\mathrm{X}$ chromosome.

\subsubsection{Differentiation Potential}

Pluripotency of hiPSCs has also been tested in assays of embryoid body formation in vitro and teratoma induction in vivo. It has been convincingly shown by several groups that hiPSCs can differentiate into all three germ layers in vitro and in vivo $[1,211]$.

\subsection{Differentiation of human Stem Cells}

In the $18^{\text {th }}$ century observations of early chick embryo development laid the foundation of the current awareness that all intra- and extraembryonic structures develop from three primary germ layers [30]. During embryogenesis, the primitive 
ectoderm of the epiblast forms all three germ layers: the mesoderm, the ectoderm and the definitive endoderm.

The in vitro differentiation potential of hESCs allows mimicking these processes. In vitro differentiation of hESCs is induced by culturing ES cells as free-floating three dimensional aggregates - so called "embryoid bodies" (EBs) [48, 64, 155].

Up to now, many protocols have been established to induce direct differentiation into a particular cell type. Directed differentiation is influenced by co-culture with different somatic cells [15, 137] and/or by supplementation of growth factors and small molecules [106, 213]. Addition of rock-inhibitor (rho-associated kinase inhibitor) Y-27632 for the first 24h improves survival of human ES single cells and promotes generation of EBs [63]. Until now, hESCs are successfully differentiated in specific cell types of all three primary germ layers including cardiomyocytes and pacemaker-like cells [91, 135, 220] or exocrine and endocrine pancreas cells [15, 27].

\subsubsection{Neuronal Differentiation}

The first step of embryonic neurogenesis is the formation of a neuron-forming (neurogenic) region. Vertebrates form a dorsal neural tube by blocking a BMP signal. During this process (neurulation), cells of the ectodermal layer convert into a columnar epithelium called the neural plate (Fig. 3). Ridges of the neural plate continue to bulge and the tops eventually come together forming the neural tube. The neural tube consists of neural precursor cells (NP, or neuroepithelial cells), able to differentiate into various specific types of neurons and glial cells. Terminal neuronal differentiation is influenced by signal proteins secreted from the ventral and dorsal sides of the neural tube. Moreover, neurons are adapted to different characters according to their "birthday" - as surrounding older neurons or glial cells also influence terminal differentiation into a specific neuronal character.

HESCs as well as hiPSCs can be differentiated into various subtypes of neurons including motoneurons, dopaminergic or serotinergic neurons, glial cells and oligodendrocytes [81, 82, 167, 174, 229, 230]. Neurons differentiated from either hESCs or hiPSCs, follow similar differentiation programs [93].

During directed neuronal differentiation of human stem cells, natural developmental procedures are imitated (Fig. 3). Usually, differentiation is induced by EB formation followed by replating cells on an adherent system. In the replated 
EB center, developing columnar cells express markers of NSCs like nestin and musashi-1 and become positive for PAX6 (paired box 6a, a key transcription factor in neurogenesis, also expressed in NSCs) $[136,229,228]$. Over the following days the columnar cells organize into neural-tube like cells and express SOX1 indicating the development of definitive neuroepithelial cells comparable to neural tube closure.

HESCs can also be differentiated without the generation of EBs. Human stem cells are therefore cultured as an adherent, monolayer culture and uniform exposure of supplements induce neuronal differentiation [83, 93]. Adherent, monolayer differentiation results in a pure population of long-term self-renewing rosette-type hESCs derived neural stem cells (It-hESNSCs). It-hESNSCs supply constant proliferation and can be cyro-preserved. Thus, these cells provide a stable source for neural differentiation with reduced batch-to-batch variations [97].

\subsubsection{Role of Media and Factors in Neural Differentiation}

Different growth factors and compounds have been used to promote differentiation of hESCs into neural precursor cells $[25,158,229]$ and to direct differentiation into a specific neuronal subtype.

\subsubsection{FGF-signaling}

In neural specification, FGF2 either can induce a "pro" neural signal at an early state $[39,228]$ or can support neural identity by acting as a BMP antagonist [39, 45]. Additionally, FGF signaling supports survival and proliferation of neuronal precursor cells [130, 143, 228]. Based on this, FGF2 is routinely used to selectively enhance NP proliferation. FGF2-induced neural progenitors appear to be a mixed population of neural precursor cells ranging from forebrain to spinal cord characteristics [229].

It is well established that pattering of $\mathrm{mid} / \mathrm{hind}$ brain is controlled by signals released in the isthmus area, mainly FGF8. Consequently, FGF8 treatment of neuronal precursor cells, before SOX1 expression, promotes the generation of midbrain progenitors and TH-positive (tyrosine hydroxylase) dopaminergic or adrenergic neurons [229].

\subsubsection{BMP signaling}


In vertebrates, a loss of BMP signaling is crucially involved in the neural induction of the ectoderm at or before gastrulation. Consequently, inhibition of BMP results in neuronal differentiation. Expression of BMP-antagonists like noggin or follistatin, released by the notochord and the floor plate, are supposed to be involved in dorso-ventral patterning of the spinal cord. With the aid of SHH (sonic hedgehog), BMPs mainly specify forebrain cells. It has been shown that inhibition of BMPsignaling via Noggin, which reduces SMAD phosphorylation, upregulates the expression of typical neural transcription factors like PAX6 or nestin [182]. A small molecule compound dorsomorphin also inhibits BMP mediated signals by selective inhibition of BMP type 1 receptors ALK2 (activin receptor like kinase 2), ALK3 and ALK6 resulting in the reduction of SMAD1/5/8 phosphorylation [134, 224]. Dual SMAD inhibition using dorsomorphin and SB431542, an inhibitor of ALK1 receptor leads to an improved "neuralization" of EBs [16].

\subsubsection{Retinoic acid signaling}

Retinoic acid (RA) treatment leads to a caudalization of neural progenitors and an expression of HOX genes, typical for a hindbrain and spinal cord phenotype [208, 229]. Furthermore, absence of RA results in missing of the posterior hindbrain and anterior spinal cord [39, 122]. RA is therefore crucial for the generation of motoneurons and other cell types located in the brainstem and the spinal cord [40, 229] and influences motoneuron subtype specification [77, 110].

\subsubsection{Sonic hedgehog signaling}

Sonic hedgehog is a crucial signal molecule in the patterning the central nervous system (CNS) during vertebrate development, secreted from the notochord cells and at later time points also from the floor plate $[53,151]$.

$\mathrm{SHH}$, a ventralizing morphogen [144], is strongly associated with the development of the floor plate and diverse ventral cell types within the neural tubes [109]. RA and $\mathrm{SHH}$ treatment result in an enrichment of neural progenitors expressing OLIG2, a transcription factor expressed by motoneuron progenitors. $\mathrm{SHH}$ is essential for the differentiation of ventral cell types including motoneurons, V2 and V3 interneurons and oligodendrocytes [81]. Moreover, SHH together with FGF8 promotes the induction of ventral midbrain progenitors as well as the generation of midbrain dopaminergic neurons $[229,230]$. 
Based on these findings, SHH, RA and FGF8 have been used to differentiate NSCs into specific neural subtypes. Interestingly, treatment of primitive neuroepithelial cells, expressing PAX6 but not SOX1, seem to be more efficient than treatment of definitive neuroepithelial cells [229].

\subsubsection{Neural differentiation of hiPSCs with variable potency}

HiPSCs provide the opportunity to produce patient specific human stem cells. However, it is necessary to investigate how reliable they can be differentiated into neurons and neuronal subtypes. Differentiation efficiency of several hiPSCs lines has been compared among each other and with hESCs lines [16, 83].

Zhang and co-workers [83] could show that different hiPSCs lines, including those with or without integrating transgenes, differentiate to neuroepithelial cells and functional glial cells following the same time course as hESCs but with an increased variability. To investigate the differences of neural differentiation potentials, Eggan and co-workers compared 16 hiPSCs lines from varying age, sex and health status with respect to pluripotency and the ability to terminally differentiate. Contrary to Zhang et al, hiPSCs lines used in that study show a similar capacity of motoneuron differentiation compared to hESCs. Although there are quantitative differences in motoneuron generation among the different hiPSCs lines, they do not reflect overall differences between hiPSCs and hESCs [16].

\subsection{Differentiation of human Stem Cells into functional Motoneurons}

Molecular interactions responsible for the specification from neural precursor cells of the neural tube to motoneurons have been well defined [6, 87]. Neural precursor cells at the ventrolateral margin of the neural tube (pMN domain) tend to become motoneurons. Motoneuron differentiation is mainly influenced by SHH and RA signaling. Ericson and colleagues [53] have shown that two critical periods of $\mathrm{SHH}$-signaling are responsible for the differentiation of definitive NP cells (PAX6+, SOX1+) into motoneurons. Here, the notochord is required for the first early $\mathrm{SHH}$ period during which naïve NP cells are instructed to become ventralized progenitors, expressing the oligodendrocyte transcription factor 2 (OLIG2, an early marker for PMNs and oligodendrocytes precursor cells) [82, 114]. This initial period is followed by a secondary SHH period mediated by the floor plate cells 
which continue into the $S$ phase of the final progenitors' cell division and influence the differentiation of ventralized progenitors to become motoneurons [53]. Next to $\mathrm{SHH}, \mathrm{RA}$ is a crucial molecule in motoneuron development. RA secreted from the paraxial mesoderm regulates the rostrocaudal organization of spinal motoneurons and is involved in motoneuron subtype specification [20,77, 110].

For motoneuron differentiation, embryonic processes are imitated. Differentiation into neuroepithelial cells is obtained by replating EBs at a low density and a chemically defined medium, neutralizing neural progenitor cells [16, 81, 114]. Neural induction results in the generation of definitive neuroepithelial cells, which are positive for PAX6 and SOX1 [146] and organize in neural tube-like rosettes. Inhibition of TGF $\beta$ signaling (via SB431542), activation of the FGF signaling as well as inhibition of the BMP pathway (via noggin or dorsomorphin) leads to an optimization of neural induction [16]. For motoneuron differentiation, neural precursor cells are predifferentiated into motoneuron progenitors by treatment with $\mathrm{RA}$, responsible for caudalizing, and with $\mathrm{SHH}$ which induces ventralizing of neural precursor cells. Motoneuron progenitors derived from human stem cells express OLIG2 and islet1 (ISL1) [52]. According to ongoing differentiation OLIG2 is downregulated [82, 114]. OLIG2 downregulation is followed by an increased expression of HB9 (motor neuron and pancreas homebox 1, specific for mature motoneurons) and NGN2 (neurogenin 2, involved in motoneuron specification [121]). Additionally motoneurons differentiated from human stem cells express other typical motoneuron markers like SMI-32 (NEFH, neurofilament, heavy polypeptide), a neurofilament characteristic for motoneurons [204] and ChAT (choline acetyltransferase), the typical transmitter of the second motoneuron in the spinal cord [81]. 


\section{Introduction}
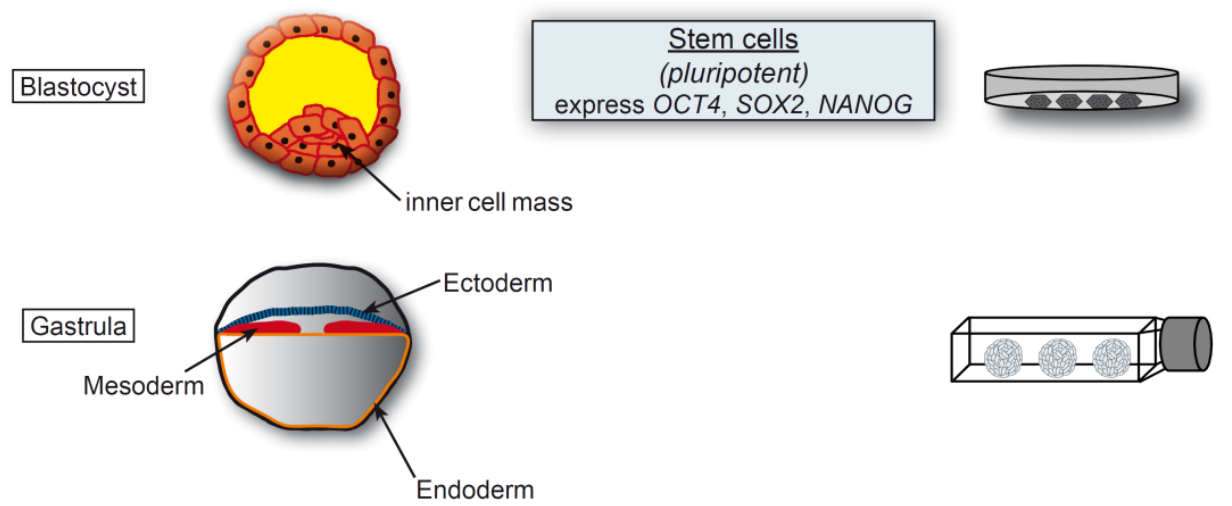

hiPSCs
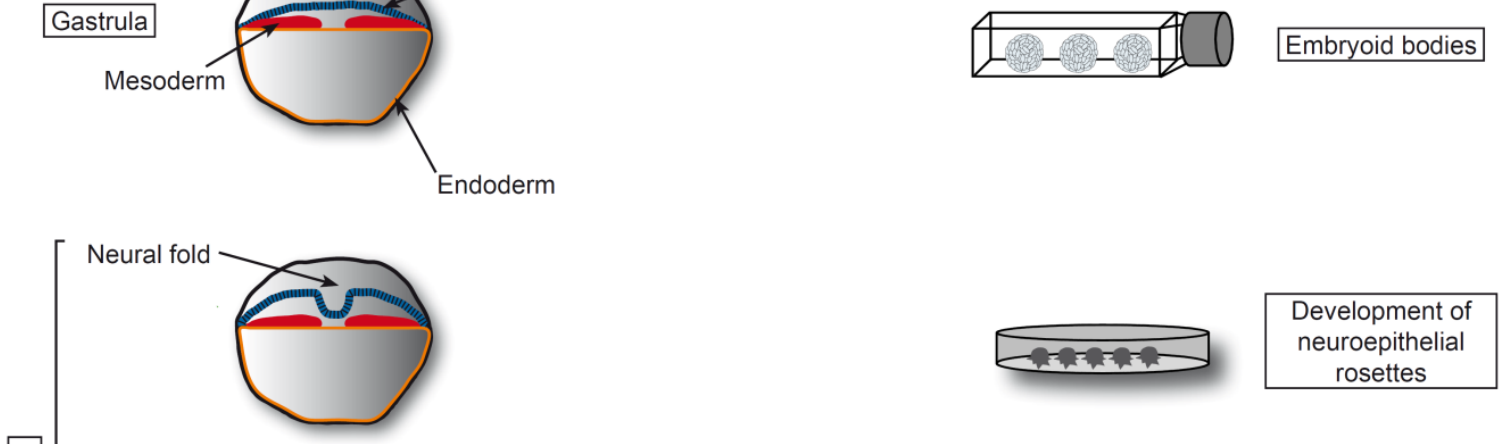

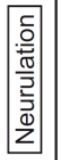
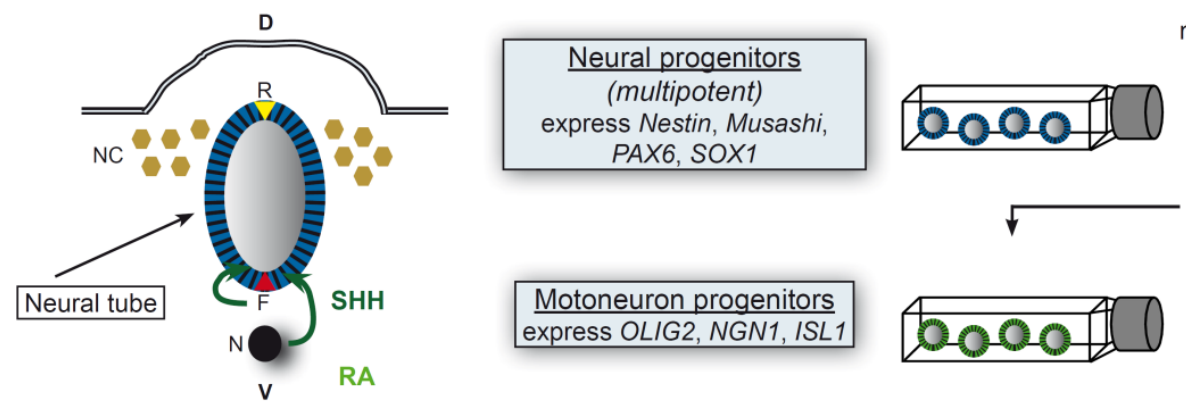

Selection of neuroepithelial rosettes
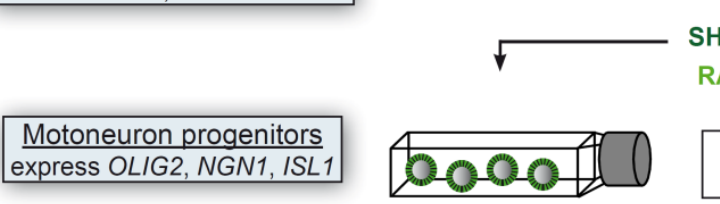

\section{Neuronal}

$\mathrm{HH}$

RA

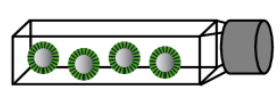

Motoneuron

progenitors

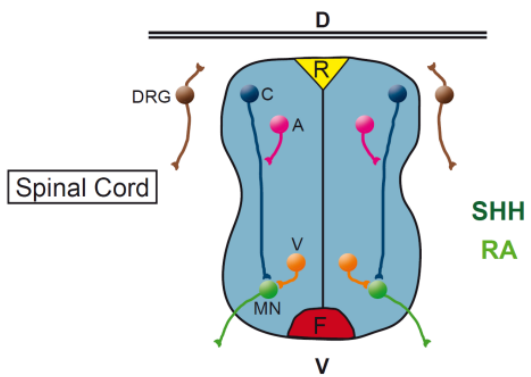

\section{postmitotic Motoneurons express $H B 9$, SMI-32}

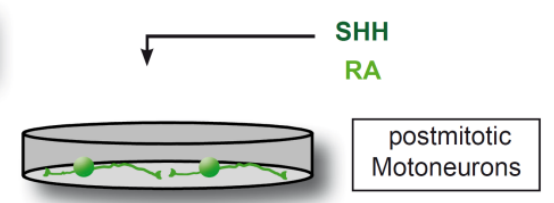

Motoneuron Development
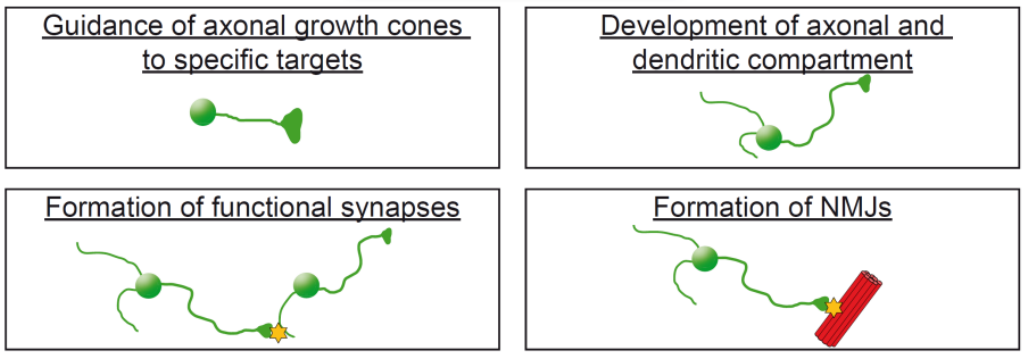

Figure 3 Development of the Spinal Cord and postmitotic Motoneurons

Overview of the development of the mammalian spinal cord and postmitotic motoneurons (right panel) versus the generation of postmitotic motoneurons from hiPSCs (left panel). The major steps of mammalian motoneuron development like the formation of the neural tube and neural precursor cells, the differentiation into motoneuron progenitors by SHH and RA signaling and the terminal differentiation into postmitotic motoneurons are also substantial parts in the differentiation protocol of hiPSCs. Major stages of the development of postmitotic motoneurons are the formation of axonal growth cones, 
the differentiation of an axonal and dendritic compartment and the generation of functional interneuronal and neuromuscular synaptic contacts.

Abbreviations: $\mathrm{D}=$ dorsal, $\mathrm{V}=$ ventral, $\mathrm{R}=$ roof plate cells, $\mathrm{NC}=$ neural crest cells, $\mathrm{F}=$ floor plate cells, $\mathrm{N}=$ notochord cells, $\mathrm{C}=$ commissural neurons, $\mathrm{A}=$ association neurons, $\mathrm{MN}=$ motoneurons, $\mathrm{V}=$ ventral interneurons, $\mathrm{DRG}=$ dorsal root ganglion neurons, $\mathrm{SHH}=$ sonic hedgehog, $\mathrm{RA}=$ retinoic acid, NMJs = neuromuscular junctions

\subsection{Maturation of Spinal Motoneurons}

Formation of a long and mature axon is a crucial step in motoneuron development and a strong prerequisite for muscle innervation. The motoneuron axon and several shorter dendrites are responsible for processing incoming signals to and from other neurons. Spinal motoneurons are able to coordinate monosynaptic as well as polysynaptic reflexes dealing with a number of different incoming signals from the periphery and from cortical motoneurons either directly or by interneurons. Consequently, the formation of functional neuronal synapses and synaptic plasticity is crucial for an appropriate motoneuronal development and function.

\subsubsection{Neuronal Synapses}

Synapses have been described for the first time by Sherrington in 1897. They are defined as a specialized structure between an axonal terminal and a dendrite or soma of another neuron, muscle cell or gland cell. Two different major forms of synapses can be distinguished - electrical synapses and chemical synapses. Electrical synapses are defined as clusters of channels connecting the cytoplasm of adjacent cells leading to electric coupling and providing transfer of small molecules. Chemical synapses are the main type in the mammalian CNS. Their complex structure is formed by a specialized axonal terminal and the postsynaptic side. Both synaptic compartments are separated by the synaptic cleft spanning a distance up to $30 \mathrm{~nm}$. According to their transmitter and the different receptors at the postsynaptic side, chemical synapses are identified as excitatory or inhibitory synapses [71]. Typical excitatory transmitters of the mammalian CNS are glutamate [65] or dopamine whereas gamma-amniobutyric (GABA) and glycine are inhibitory transmitters [94]. Glutamate is also the typical transmitter between the upper and lower motoneurons.

Additional morphological investigation of synapses resulted in further classifications, Gray type 1 and Gray type 2 [69]. Type 1 synapses are described as asymmetric synapses and are usually excitatory. Typical characteristics are a relatively wide $(\sim 20 \mathrm{~nm})$ synaptic cleft, small and round presynaptic vesicles and 
an obvious postsynaptic density (PSD). Type 2 or symmetric synapses are normally inhibitory with a less defined PSD and pleomorphic synaptic vesicles.

The presynaptic side, formed by an axonal terminal, usually contains the active zone (AZ) responsible for transmitter release. Neurotransmitter containing synaptic vesicle fusion and release is strongly regulated by intracellular calcium $\left(\mathrm{Ca}^{2+}\right)$ concentrations [44]. Mobilization and anchoring of synaptic vesicles is arranged by an electron-dense matrix known as the cytomatrix at the active zone (CAZ) [44]. Presynaptic proteins including different proteins responsible for the fusion of synaptic vesicles, cytoskeletal molecules, ion channels, scaffolding proteins and cell adhesion molecules are also organized by the CAZ. Next to others, Bassoon (BSN) [199] and Piccolo (PCLO) [56] are highly enriched in the presynaptic AZ. Both are very large structure proteins of the presynaptic side involved in $A Z$ architecture and functioning [56, 225]. AZ associated proteins are probably generated in the Golgi apparatus and transported to the presynaptic membrane. Those Piccolo-Bassoon transport vesicles (PTVs) or dense-core vesicles (DCVs) are proposed to be essential in the initial step of synaptogenesis, when a defined number of PTVs are inserted into the presynaptic membrane $[67,171]$.

The corresponding postsynaptic density is an electron dense mashwork underneath the postsynaptic membrane. The PSD is defined by a pool of several hundred different proteins including membrane-bound receptors, cell adhesion proteins, proteins of different signaling cascades, and cytoskeletal components $[13,107]$.

Additionally, the PSD is characterized by specific scaffolding proteins like SAP90/PSD-95 [162, 171], a member of the MaGuK family and a direct interaction partner of N-methyl-D-aspartate (NMDA) receptors.

In the interface between membrane-bound receptors and the actin cytoskeleton master scaffolding proteins of the ProSAP/SHANK family are localized $[12,11]$. ProSAP/SHANK proteins are multidomain proteins and represent an integral component of connections between glutamate receptors complexes or parts of various signaling cascades at the PSD and the actin-based cytoskeleton $[12,171]$. ProSAP1/SHANK2 and, especially, ProSAP2/SHANK3 are early structural elements of the developing synapse and both influence synaptic plasticity $[11,67]$. Moreover the sterile alpha motif (SAM) domain of ProSAP2/SHANK3 is able to form large platforms within the PSD which are indicated to be a core component of 
the PSD [4]. In contrast to ProSAP1/SHANK2 and ProSAP2/SHANK3, SHANK1 is the last ProSAP/SHANK family member appearing in newly forming synapses. This indicates that Shank1 is mainly responsible for synapse maturation rather than synaptogenesis [68].

Homer, another protein of the PSD and a binding partner of the prolin-rich region of ProSAP/SHANK family, accumulates with metabotrophic glutamate receptors (mGluRs) in the PSD. In addition HOMER1 interacts with the inositiol triphosphate receptor (IP3R) of the dendritic spine apparatus to set the scene for glutamatedependent calcium signaling $[161,200]$.

\subsubsection{Neuromuscular Junctions}

Neuromuscular junctions define the connection of the axonal terminal of a motoneuron with the motor end plate, the highly-excitable region of the muscle fiber plasma membrane required for the initiation of action potentials. The NMj uses different transmitters in different species: for example acetylcholine (ACh) in vertebrates and glutamate in Drosophila, both are excitatory and cause muscle contraction [215].

The post synaptic apparatus of NMjs contains a high-density web of numerous neurotransmitter receptors - strongly associated with different extracellular, transmembrane and cytoplasmic proteins [165]. In contrast to neuronal synapses the sarcolemma is highly infolded and the basal lamina is very prominent.

The molecular composition of the neuromuscular junction (NMj) is crucial for its function and maintenance whereas dysregulation of endplate physiology is considered to be involved in denervation of the muscle cells and subsequent motoneuron degeneration [46, 47].

Scaffolding molecules of the postsynaptic site of NMjs most importantly cluster the acetylcholine receptors which transduce a sodium influx into the cell upon binding of the neurotransmitter acetylcholine [35, 215]. The development of the NMjs is characterized by distinct differentiation steps [164]. The initial development of muscle cells is independent from neuronal innervation, but the motoneuron requires reciprocal signals from the muscle cell to initiate and uphold NMjs. Furthermore, these signals seem to trigger neuronal survival or intramuscular branching. Acetylcholine released from the arriving axonal nerve terminal finally determines the formation of a functional NMj [131]. On the postsynaptic site, nerve 
independent clustering of acetylcholine receptors, the so called prepatterning is a crucial prerequisite for initiation and proper formation of a neuromuscular connection with the growing axon.

\subsubsection{Synaptogenesis}

Synaptogenesis is a complex process requiring a highly specific arrangement of proteins at both sides of the synapse. Mammalian synaptogenesis is tightly associated to neuronal differentiation resulting in an increased formation of preand postsynaptic proteins, directed transport and a specific assembly of the preand postsynaptic complex. However, the exact events that are responsible for synapse induction and its intrinsic composition are still barely understood [62]. Synaptogenesis can be divided into distinct steps of development, but the exact events that induce synapse formation have not yet been completely resolved.

Formation of filopodia-like structures along the dendrites indicates the development of an initial contact side [62] followed by the induction of the pre-and postsynaptic specialization. Ongoing synapse specification leads to an increased transport and accumulation of pre- and postsynaptic proteins at both sides of the synaptic contact.

There is increasing evidence that the presynaptic specialization seems to be the first to be assembled and that assembly mechanisms are different compared to the postsynapse [62]. Presynaptic specialization is mainly caused by the fusion of DCVs with the presynaptic membrane during synaptogenesis. In contrast, postsynaptic proteins are transported and recruited individually to the synaptic sides, where they include into a macromolecular complex [107]. However, recent studies reported on a trimetric complex containing Shank1 and Neuroligin, which could be a prebuilt postsynaptic precursor complex [66], [107]. Synapses can be maintained over years but still remain the ability to change in response to either use or disuse of transmission over synaptic pathways. This ability is called "synaptic plasticity" and is a crucial prerequisite of learning and memory.

\subsection{Amis of the thesis}

Motoneurons differentiated from patient specific human iPSCs offer a dynamic model for analyzing and studying pathological processes that lead to human 
motoneuron degeneration and even may serve as a template for pharmaceutical screening. Comparison of patient specific motoneurons with motoneurons from healthy controls depends on the analysis of human cell differentiation, morphology and protein expression under physiological in vitro conditions. With respect to the nervous system it is essential to understand the exact time course and stage specific patterning of neural differentiation and synaptogenesis. For the rodent brain or e.g. primary neurons in culture many developmental aspects have already been elucidated [67, 107]. Next to inter-neuronal synaptogenesis, the formation of NMjs is crucial for motoneuron function and an impaired assembly of NMjs results in motoneuron degeneration [139].

The aim of this doctoral thesis was to characterize the distinct steps of the development of both inter-neuronal and neuromuscular contacts in hiPSC-derived motoneurons. Moreover, all critical points of motoneuron development including neurite development, cell polarization and axon formation have been investigated. 


\section{Material and Methods}

\subsection{Materials}

\subsubsection{Tools and Machines}

Cell scraper

Cellculture T75 flasks

Cellculture T175 flasks

Cellculture plates 24 well

Cellculture plates 6 well

Cellculture dishes $94 \mathrm{~mm}$

Cellculture low-attachment T75 flasks

Centrifuge Multifuge

Centrifuge Avanti J-25

Cover glasses $13 \mathrm{~mm}$

Dissection set of instruments

Eppendorf cups

Falcon Tubes 15 / 50 ml

Freezer $-20^{\circ} \mathrm{C}$

TPP, Trasadingen, Switzerland

Nunc, Wiesbaden, HE, Germany

Nunc, Wiesbaden, HE, Germany

Nunc, Wiesbaden, HE, Germany

Nunc, Wiesbaden, HE, Germany

Nunc, Wiesbaden, HE, Germany

Nunc, Wiesbaden, HE, Germany

Heraus Holding, Hanau, HE, Germany

Beckmann, Krefeld, NRW, Germany

Menzel, Braunschweig, NI, Germany

VWR, Darmstadt, HE, Germany

Eppendorf, Hamburg, HH, Germany

Nunc, Wiesbaden, HE, Germany

BSH, Giengen, BWM Germany

Freezer $-80^{\circ} \mathrm{C}$

BSH, Giengen, BWM Germany

Freezer tubes $1.8 \mathrm{ml}$

Nunc, Wiesbaden, HE, Germany

Fridge $4^{\circ} \mathrm{C}$

BSH, Giengen, BW, Germany

$\mu$ dishes, $35 \mathrm{~mm}$, treated

Ibidi, Munic, BY, Germany

Ice Machine

Scotsman, Vernon Hilly, IL, USA

Incubators $37^{\circ}$

Heraus Holding, Hanau, Germany

Microscope Axiokope 2 mot plus

Microscope Axiovert 25

Microwave

Zeiss, Oberkochen, BW, Germany

Zeiss, Oberkochen, BW, Germany

BSH, Giengen, BW, Germany

Neubauer counting chamber

Brandt, Wertheim, BW, Germany

Nylon cell sieve $125 \mu \mathrm{m}$

Falson, Gräfelfing, Munic, Germany

Object slides

Pasteur pipettes

Menzel, Braunschweig, NI, Germany

$\mathrm{pH}$ meter inoLab Level 1

Brandt, Wertheim, BW, Germany

WTW, Weilheim, BY, Germany

Pipetboy

Hirschmann, Eberstadt, BW, Germany 
Pipette tips $10 \mu \mathrm{l} / 200 \mu \mathrm{l} / 1 \mathrm{ml} / 5 \mathrm{ml}$ RNA Pipette tips $10 \mu \mathrm{l} / 100 \mu \mathrm{l} / 1 \mathrm{ml}$ Pipettes

RotorGene Q System

RT-PCR tubes / stripes

PCR-plate

Sterile bench Laminar flow

Sterile filters 0.22 / $0.45 \mu \mathrm{m}$

Tabletop Combispin FVL-2400

Tabletop centrifuge Biofuge pico

Thermomixer comfort

Transmission electron microscope

Vortexer
Eppendorf, Hamburg, HH, Germany Eppendorf, Hamburg, HH, Germany Eppendorf, Hamburg, HH, Germany Qiagen, Hilden, NRW, Germany Qiagen, Hilden, NRW, Germany Qiagen, Hilden, NRW, Germany Nunc, Wiesbaden, HE, Germany Schleicher\&Schuell, Dassel, NI,Germany Peglab, Erlangen, BY, Germany Heraus Holding, Hanau, HE, Germany Eppendorf, Hamburg, HH, Germany 10 Zeiss, Oberkochen, BW, Germany VWR, Darmstadt, HE, Germany

\subsubsection{Laboratory Animals}

For the preparation of primary motoneurons and rat embryonic fibroblasts (REFs) day E14 Sprague Dawley rat embryos were used. For the preparation of primary mouse myotubes Black six mouse newborn pubs (day 1 -3) were used. Pregnant animals were killed by an overdose of $\mathrm{CO}_{2}$. All animal experiments were performed in compliance with the guidelines for the welfare of experimental animals issued by the Federal Government of Germany, the National Institutes of Health and the Max Planck Society (Nr. O.103).

\subsubsection{Chemical Substances for Cell Culture}

\subsubsection{Cell culture media}

- PBS (phosphate buffered saline), (Invitrogen, Carlsbad, CA, USA)

- PBS - - (phosphate buffered saline without calcium and magnesium), (Invitrogen)

- HBSS - - (Hank's buffered salt solution without calcium and magnesium), (Invitrogen)

- Opti-MEM, (Invitrogen)

Stored at $4^{\circ} \mathrm{C}$.

- DMEM 2x, (Invitrogen) 
DMEM powder was dissolved in $450 \mathrm{ml}$ destilled $\mathrm{H}_{2} \mathrm{O}$ and $3.7 \mathrm{~g}$ sodium bicarbonate were added ( $\mathrm{pH}$ 7.7). Solution was sterile filtered and stored at $4^{\circ} \mathrm{C}$.

- DMEM/F12, (Invitrogen)

Basalmedia for neuronal differentiation. Stored at $4^{\circ} \mathrm{C}$.

- Knockout DMEM, (Invitrogen)

Basalmedia for hiPSCs medium. Stored at $4^{\circ} \mathrm{C}$.

- Knockout DMEM/F12, (Invitrogen)

Basalmedia of primary motoneuron cultures. Stored at $4^{\circ} \mathrm{C}$.

- HAM F12, (Invitrogen)

Basalmedia for primary and human myotubes cultures. Stored at $4^{\circ} \mathrm{C}$.

- mTeSR1, (Stemcell Technologies, Vancouver, CA, USA)

Media for hiPSCs under feeder-free conditions. Stored at $4^{\circ} \mathrm{C}$.

- Epilife \& HKGS supplement, (Invitrogen)

Media for keratinocytes culture. Supplemented with HKGS and stored at $4^{\circ} \mathrm{C}$.

- Fetal bovine serum (FBS), (Invitrogen)

Preparation of $50 \mathrm{ml}$ aliquots and stored at $-20^{\circ} \mathrm{C}$.

- Horse serum, (Invitrogen)

Heat inactivation at $56^{\circ} \mathrm{C}$ for $30 \mathrm{~min}$. Preparation of $50 \mathrm{ml}$ aliquots and stored at $-20^{\circ} \mathrm{C}$.

- Goat serum, (Chemicon, Billerica, MA, USA)

Perparation of $20 \mathrm{ml}$ aliquots and stored at $-20^{\circ} \mathrm{C}$.

- Knockout Serum Replacement, (Invitrogen)

Preparation of $50 \mathrm{ml}$ aliquots and stored at $-20^{\circ} \mathrm{C}$.

- Non-essential-aminoacids (NEAA), 100x liquid, (Invitrogen)

Preparation of $10 \mathrm{ml}$ aliquots and stored at $4^{\circ} \mathrm{C}$.

- GlutaMax ${ }^{\text {TM }}$ 100x liquid, (Invitrogen)

Preparation of $10 \mathrm{ml}$ aliquots and stored at $-20^{\circ} \mathrm{C}$.

- Antibiotic Antimycotic, 100x liquid, (Invitrogen)

Preparation of $10 \mathrm{ml}$ aliquots and stored at $-20^{\circ} \mathrm{C}$.

- HESCs-qualified- $\beta$-mercaptoethanol, (Millipore, Billerica, MA, USA)

Compound of hiPSCs medium. Stored at $4^{\circ} \mathrm{C}$. 
- Sodium Pyruvat, 100x liquid, (Invitrogen)

Stored at $4^{\circ} \mathrm{C}$.

- HEPES-buffer, 100x liquid, (Invitrogen)

Stored at $4^{\circ} \mathrm{C}$.

\subsubsection{Freezing Media}

- Stem cell mFreSR, (Stemcell Technologies)

$1 \mathrm{ml} /$ freezing tube was used. Stored at -20 .

- Synth-a-Freeze, (Invitrogen)

$1 \mathrm{ml} /$ freezing tube was used. Stored at $4^{\circ} \mathrm{C}$

\subsubsection{Supplements}

- Ascorbic Acid, (Sigma-Aldrich, St. Louis, MO, USA)

For motoneuron differentiation media a $200 \mu \mathrm{g} / \mathrm{ml}$ stock solution was prepared in PBS. For hiPSCs media a $50 \mathrm{mg} / \mathrm{ml}$ stock solution was prepared. Stored at $-20^{\circ} \mathrm{C}$.

- B27 without Vit. A, (Invitrogen)

B27 supplement was used 1:50 and stored at $-20^{\circ} \mathrm{C}$ and for short-time storage at $4^{\circ} \mathrm{C}$. Aliquots were protected for light.

- cAMP, (Sigma-Aldrich)

A $1 \mathrm{mM}$ stock solution was prepared. $4.914 \mathrm{mg}$ of cAMP were dissolved in $10 \mathrm{ml}$ sterilized water. Aliquot and stored at -80 .

- Chick embryo extract (CEE), (US biologicals, Swampscotts, MA, USA) Stored at $4^{\circ} \mathrm{C}$.

- Heparin,(Sigma-Aldrich)

A $1 \mathrm{mg} / \mathrm{ml}$ stock solution was prepared. $10 \mathrm{mg}$ of heparin were dissolved in $10 \mathrm{ml}$ DMEM, aliquot and stored at $-80^{\circ} \mathrm{C}$.

- Insulin,(Sigma-Aldrich)

A $1 \mathrm{mg} / \mathrm{ml}$ stock solution was prepared. $100 \mathrm{mg}$ of insulin were dissolved in $12 \mathrm{ml} \mathrm{HCL}(1 \mathrm{~N})$ and $88 \mathrm{ml}$ sterilized water. Aliquot and stored at $-20^{\circ} \mathrm{C}$.

- Leukemia-inhibitory factor (LIF), (Chemicon, ESGRO)

$10^{7}$ units were solved in $100 \mathrm{ml}$ PBS. $2 \mathrm{ml}$ aliquots were prepared and stored at $-20^{\circ}$. 


\section{- Hormone-mix}

Detailed preparation is describes in 2.2.5.1. Aliquot and stored at $-20^{\circ} \mathrm{C}$.

- Progesterone, (Sigma-Aldrich)

A $2 \mathrm{mM}$ stock solution was prepared. $1 \mathrm{mg}$ of progesterone was dissolved with $1.59 \mathrm{ml} 95 \% \mathrm{EtOH}$ and aliquot $40 \mu \mathrm{l}$ per tube. Stored at $-20^{\circ} \mathrm{C}$.

- Purmorphamine, (Calbiochem, Gibbstown, NJ, USA)

A $10 \mathrm{mM}$ stock solution was prepared. $5 \mathrm{mg}$ of purmorphamine were dissolved in $480 \mu \mathrm{l} 100 \%$ ethanol and $480 \mu \mathrm{l}$ DMSO. Aliquot and stored at $80^{\circ} \mathrm{C}$. The working concentration range of purmorphamine is very narrow, so the stock solution was prepared as accurately as possible. When adding the stock solution into the culture medium the smallest tips and a wellcalibrated pipette had been used.

- Recombinant human FGF2 (Peprotech, Rocky Hill, NJ, USA)

A $25 \mu \mathrm{g} / \mathrm{ml}$ stock solution was prepared. $50 \mu \mathrm{g} / \mathrm{ml} \mathrm{FGF2}$ was resolved in $2 \mathrm{ml}$ of $5.5 \mathrm{mM}$ Tris buffer, $\mathrm{pH}$ 7.6. Stored at $-20^{\circ} \mathrm{C}$.

- Recombinant human BDNF, (Peprotech)

A $10 \mu \mathrm{g} / \mathrm{ml}$ stock solution was prepared. $50 \mu \mathrm{g} / \mathrm{ml}$ of BDNF was resolved in $50 \mu \mathrm{l}$ of sterilized PBS - - with $0.1 \% \mathrm{BSA}$. Stored at $-20^{\circ} \mathrm{C}$.

- Recombinant human GDNF, (Peprotech)

A $10 \mu \mathrm{g} / \mathrm{ml}$ stock solution was prepared. $50 \mu \mathrm{g} / \mathrm{ml}$ of GDNF was resolved in $50 \mu \mathrm{l}$ of sterilized PBS - - with $0.1 \% \mathrm{BSA}$. Stored at $-20^{\circ} \mathrm{C}$.

- Recombinant human IGF-2, (Peprotech)

A $10 \mu \mathrm{g} / \mathrm{ml}$ stock solution was prepared. $50 \mu \mathrm{g} / \mathrm{ml}$ of GDNF was resolved in $50 \mu \mathrm{l}$ of sterilized PBS - - with $0.1 \% \mathrm{BSA}$. Stored at $-20^{\circ} \mathrm{C}$.

- Recombinant human EGF, (Peprotech)

A $10 \mu \mathrm{g} / \mathrm{ml}$ stock solution was prepared. $50 \mu \mathrm{g} / \mathrm{ml}$ of EGF was resolved in $50 \mu \mathrm{l}$ of sterilized PBS - - with $0.1 \% \mathrm{BSA}$. Stored at $-20^{\circ} \mathrm{C}$.

- Retinoid Acid (RA), (Sigma-Aldrich)

A $100 \mathrm{mM}$ stock solution was prepared. $50 \mathrm{mg}$ RA were dissolved in 1.67 $\mathrm{ml}$ of DMSO and aliquot $50 \mu \mathrm{l}$ per tube. The concentrated sock was stored at $-80^{\circ} \mathrm{C}$. To prepare a $1 \mathrm{mM}$ working solution each concentrated aliquot was diluted with $4.95 \mathrm{ml}$ of $100 \% \mathrm{EtOH}$. Stored at $-20^{\circ} \mathrm{C}$. 
- Rock-inhibitor Y-27632, (Ascent Scientific, Avonmouth, BS, UK)

Rock-inhibitor was resolved with sterile $\mathrm{H}_{2} \mathrm{O}$ to an end concentration of 10 $\mathrm{mM}$ and stored at $-20^{\circ} \mathrm{C}$.

- Sodium Selenite (SoSe), (Sigma Aldrich)

A $3 \mathrm{mM}$ Stock solution was prepared. $1 \mathrm{mg}$ Natriumselenit was dissolved with $1.93 \mathrm{ml}$ distillated $\mathrm{H}_{2} \mathrm{O}$ and aliquot $40 \mu \mathrm{l}$ per tube. Stored at -20 .

\subsubsection{Coatings}

- Collagen IV, (Sigma-Aldrich)

Collagen IV was diluted in $2.5 \mathrm{ml} 0.25 \%$ acetic acid over night at $4^{\circ} \mathrm{C}$. Stored at $4^{\circ} \mathrm{C}$. For coating, collagen was diluted in PBS - - 1:100 and $500 \mu \mathrm{l}$ were added to each well and incubated for $1 \mathrm{~h}$ at $37^{\circ} \mathrm{C}$. Coating was followed by three washing steps with PBS - - and $70 \%$ ethanol.

- $0.1 \%$ gelantine, (Sigma-Aldrich)

Gelantine powder was diluted with PBS - - and autoclaved. Stored at -20. Coating was performed for 10-20 min at room temperature.

- Laminin (Roche, Basel, Switzerland)

$500 \mu \mathrm{l}$ aliquots were performed and stored at $4^{\circ} \mathrm{C}$. For coating, Laminin was diluted 1:25 (20 $\mathrm{gg} / \mathrm{ml})$ in DMEM/F12 and incubated for $1 \mathrm{~h}$ at $37^{\circ} \mathrm{C}$.

- HESCs-qualified matrigel (hESC-MG), (BD Biosciences, Franklin lakes, NJ, USA)

hESC-MG was diluted with DMEM/F12 according to manufactures protocol. Aliquots were carefully thawed on ice at $4^{\circ} \mathrm{C}$ over night. For coating, $500 \mu \mathrm{l}$ were added to each well and incubated for $1 \mathrm{~h}$ at room temperature.

- Matrigel for keratinocytes (k-MG), (BD Biosciences)

$\mathrm{K}-\mathrm{MG}$ was diluted with Epilife 1:2. Aliquots were stored at $-20^{\circ} \mathrm{C}$. For coating aliquots were further diluted in Epilife 1:10 or 1:5.

- Poly-L-Ornithin, (Sigma-Aldrich)

For coating, $500 \mu \mathrm{l}$ were added to each well and incubated for $1 \mathrm{~h}$ at $37^{\circ} \mathrm{C}$ or over night at $4^{\circ} \mathrm{C}$. Coating was followed by three washing steps with PBS. 


\subsubsection{Enzymes}

- Alfazyme, (PAA Labroratiories $\mathrm{GmbH}$, Pasching, Austria)

$10 \mathrm{ml}$ aliquots were stored at $-20^{\circ} \mathrm{C}$.

- StemPro-Accutase, (Invitrogen)

$10 \mathrm{ml}$ aliquots were stored at $-20^{\circ} \mathrm{C}$.

- DNAse I, (Invitrogen)

$1 \mu \mathrm{l}$ DNAse was diluted in $2 \mathrm{ml} \mathrm{HBSS}$--. Aliquot and stored at $-20^{\circ} \mathrm{C}$.

- HESCs-qualified dispase (hESC-dispase), (Stemcell Technologies)

$2 \mathrm{ml}$ aliquots were stored at $-20^{\circ} \mathrm{C}$. For use $2 \mathrm{ml}$ aliquots were diluted with 8 $\mathrm{ml}$ DMEM F12 with an end concentration of $1 \mathrm{mg} / \mathrm{ml}$.

- Dispase for keratinocytes (k-dispase), (BD Bioscience)

$10 \mathrm{ml}$ aliquots were stored at $-20^{\circ} \mathrm{C}$.

- Pronase, (Roche)

A $2 \%$ stock solution was prepared by dissolving pronase powder in destilled $\mathrm{H}_{2} \mathrm{O} .500 \mu \mathrm{l}$ aliquots were prepared and stored at $-20^{\circ} \mathrm{C}$.

- TrypLE Express, (Invitrogen)

Stored at $4^{\circ} \mathrm{C}$.

\subsubsection{Gradients}

- Percoll, (Sigma-Aldrich)

A $90 \%$ stock solution was prepared. $18 \mathrm{ml}$ percoll were sterile filtered and dissolved with $2 \mathrm{ml} 10 x P B S$ and $0.15 \mathrm{ml} \mathrm{HCL}(1 \mathrm{~N})$. PH was at 7.2. Stored at $4^{\circ} \mathrm{C}$.

- Nycodens $60 \%(w / v)$ Solution in water, (Serva, Heidelberg, BW, Germany)

Stored at $4^{\circ} \mathrm{C}$.

\subsubsection{Kits}

- QIAmp DNA Mini Kit, (Qiagen, Hilden, NRW, Germany) Used for DNA isolation.

- RNAeasy Mini Kit, (Qiagen) Used for mRNA isolation.

- Rotor-Gene SYBR Green RT-PCR Kit, (Qiagen) Used for quantitative real time PCR. 
- StemLite Pluripotency Kit, (Cell Signaling, Danvers, MA, USA) Used for immunocytochemistry analysis.

- Lenti-X ${ }^{\text {TM }}$ qRT-PCR Titration Kit, (Clontech, Mountain View, CA, USA) Used for quantification of the virion content of lentiviral supernatant.

- NucleoSpin Viral RNA Isolation Kit, (Macherey-Nagel, Düren, NRW, Germany)

Used for isolation of viral genomic RNA. 


\subsubsection{Antibodies}

\subsubsection{Primary Antibodies}

\section{Table 1: Primary Antibodies}

\begin{tabular}{|c|c|c|c|}
\hline Antibody & Isotype & Source & Dilution \\
\hline a-Actinin & $\begin{array}{l}\text { monoclonal } \\
\text { mouse IgG }\end{array}$ & Sigma-Aldrich & $1: 150$ \\
\hline $\begin{array}{c}\text { AFP } \\
\text { ( } \alpha \text {-feto-protein) }\end{array}$ & $\begin{array}{l}\text { polyclonal } \\
\text { goat IgG }\end{array}$ & Santa Cruz & $1: 100$ \\
\hline$\alpha$-Bungarotoxin & NA & Invitrogen & $1: 500$ \\
\hline $\begin{array}{c}\text { GFAP } \\
\text { (Glial fibrillary acidic protein) }\end{array}$ & $\begin{array}{l}\text { monoclonal } \\
\text { mouse IgG }\end{array}$ & Sigma-Aldrich & $1: 6000$ \\
\hline HOMER1 & $\begin{array}{l}\text { polyclonal } \\
\text { rabbit IgG }\end{array}$ & SYSY & $1: 500$ \\
\hline HOMER1 & $\begin{array}{c}\text { polyclonal } \\
\text { guinea pig lgG }\end{array}$ & SYSY & $1: 500$ \\
\hline HB9 / MNR2 & $\begin{array}{l}\text { polyclonal } \\
\text { mouse IgG }\end{array}$ & DSHB & $1: 50$ \\
\hline Islet 1 & $\begin{array}{l}\text { polyclonal } \\
\text { rabbit IgG }\end{array}$ & Abcam & $1: 200$ \\
\hline MAP2 & $\begin{array}{l}\text { polyclonal } \\
\text { rabbit lgG }\end{array}$ & Millipore & $1: 500$ \\
\hline $\begin{array}{c}\text { MHC class I } \\
\text { (myosin heavy chain) }\end{array}$ & $\begin{array}{l}\text { polyclonal } \\
\text { rabbit lgG }\end{array}$ & Abcam & $1: 500$ \\
\hline $\begin{array}{c}\text { MBP 1 } \\
\text { (myelin basic protein 1) }\end{array}$ & $\begin{array}{c}\text { polyclonal } \\
\text { chicken IgG }\end{array}$ & Chemicon & $1: 100$ \\
\hline phospho-lkB- $\alpha$ & $\begin{array}{l}\text { monoclonal } \\
\text { mouse IgG }\end{array}$ & Cell Signaling & $1: 200$ \\
\hline Piccolo & $\begin{array}{l}\text { polyclonal } \\
\text { rabbit IgG }\end{array}$ & SYSY & $1: 500$ \\
\hline ProSAP1 & rabbit IgG & [166] & $1: 600$ \\
\hline SMI-32 (NF-H) & $\begin{array}{l}\text { monoclonal } \\
\text { mouse IgG }\end{array}$ & Covance & $1: 1000$ \\
\hline Synaptophysin & $\begin{array}{l}\text { polyclonal } \\
\text { rabbit IgG }\end{array}$ & Abcam & $1: 500$ \\
\hline Synaptophysin & $\begin{array}{c}\text { polyclonal } \\
\text { guinea pig IgG }\end{array}$ & SYSY & $1: 500$ \\
\hline $\begin{array}{c}\text { Tuj1 } \\
\text { (tubulin beta 3) }\end{array}$ & $\begin{array}{c}\text { polyclonal } \\
\text { chicken IgG }\end{array}$ & Millipore & $1: 1000$ \\
\hline $\begin{array}{c}\text { Tuj1 } \\
\text { (tubulin beta 3) }\end{array}$ & $\begin{array}{l}\text { polyclonal } \\
\text { rabbit IgG }\end{array}$ & Covance & $1: 1500$ \\
\hline $\begin{array}{c}\text { VAChT } \\
\text { (vesicular acetylcholine } \\
\text { transporter) }\end{array}$ & $\begin{array}{c}\text { polyclonal } \\
\text { guinea pig lgG }\end{array}$ & Chemicon & $1: 500$ \\
\hline StemLite Pluripotency Kit & & Cell Signaling & \\
\hline
\end{tabular}

Abcam, Cambridge, CB, UK, Cell Signaling, Danvers, MA, USA, Covance, Princton, NJ, USA, Invitrogen, Carlsbad, CA, USA, Millipore, Billerica, MA, USA, Santa Cruz, Santa Cruz, CA, USA, Sigma-Aldrich, St. Louis, MO, USA, Synaptic Systems (SYSY), Goeppingen, BW, Germany 


\subsubsection{Secondary Antibodies}

Table 2: Secondary Antibodies

\begin{tabular}{|c|c|c|c|}
\hline Antibody & type & Source & Dilution \\
\hline Alexa Fluor 488 & anti mouse IgG & Invitrogen & $1: 500$ \\
\hline Alexa Fluor 488 & anti rabbit IgG & Invitrogen & $1: 500$ \\
\hline Alexa Fluor 488 & anti goat IgG & Invitrogen & $1: 500$ \\
\hline Alexa Fluor 488 & anti guinea pig IgG & Invitrogen & $1: 500$ \\
\hline Alexa Fluor 568 & anti mouse IgG & Invitrogen & $1: 500$ \\
\hline Alexa Fluor 568 & anti mouse IgM & Invitrogen & $1: 500$ \\
\hline Alexa Fluor 568 & anti rabbit IgG & Invitrogen & $1: 500$ \\
\hline Alexa Fluor 568 & anti goat IgG & Invitrogen & $1: 500$ \\
\hline Alexa Fluor 568 & anti guinea pig IgG & Invitrogen & $1: 500$ \\
\hline Alexa Fluor 647 & anti mouse IgG & Invitrogen & $1: 500$ \\
\hline Alexa Fluor 647 & anti rabbit IgG & Invitrogen & $1: 500$ \\
\hline Alexa Fluor 647 & anti guinea pig IgG & Invitrogen & $1: 500$ \\
\hline Alexa Fluor 647 & anti chicken IgG & Invitrogen & $1: 500$ \\
\hline $\begin{array}{c}\text { Aminomethylcoumarin Acetate } \\
\text { (AMCA, blue) }\end{array}$ & anti mouse IgG & Dianova & $1: 200$ \\
\hline $\begin{array}{c}\text { Aminomethylcoumarin Acetate } \\
\text { (AMCA, blue) }\end{array}$ & anti rabbit IgG & Dianova & $1: 200$ \\
\hline $\begin{array}{c}\text { Aminomethylcoumarin Acetate } \\
\text { (AMCA, blue) }\end{array}$ & anti goat IgG & Dianova & $1: 200$ \\
\hline $\begin{array}{c}\text { Aminomethylcoumarin Acetate } \\
\text { (AMCA, blue) }\end{array}$ & anti guinea pig IgG & Dianova & $1: 200$ \\
\hline $\begin{array}{c}\text { 4',6-diamidino-2-phenylindole } \\
\text { (DAPI) }\end{array}$ & nuclei staining & Invitrogen & NA \\
\hline
\end{tabular}

Dianova, Hamburg, HH, Germany, Invitrogen, Carlsbad, CA, USA

\subsection{Cell Culture}

Cell culturing was performed under sterile conditions under a sterile bench and the incubation took place in an incubator at $37^{\circ} \mathrm{C}$ in a water saturated atmosphere of 5 $\% \mathrm{CO}_{2}$ and $95 \%$ respectively $5 \% \mathrm{O}_{2}$. All used media had been warmed up using a $37^{\circ} \mathrm{C}$ water bad.

The use of human material is approved by the ethical committee of the UIm University (Nr.0148/2009) and in compliance with the guidelines of the Federal Government of Germany and the Declaration of Helsinki concerning Ethical Principles for Medical Research Involving Human Subjects.

\subsubsection{Culture of human Keratinocytes}

\subsubsection{Plucking of human hair and initially outgrowth of keratinocytes}

Human hairs were plucked from head hair and cultured as previously described [1]. T75 flasks were coated with k-MG (Epilife, 1:10) for $1 \mathrm{~h}$ at $37^{\circ} \mathrm{C}$. The outer rout 
sheet was carefully cut off from the hair and put into a drop of 1:5 k-MG. After two hours $1 \mathrm{ml}$ of MEF conditioned medium (CM) supplemented with $50 \mu \mathrm{g} / \mathrm{ml}$ ascorbic acid, $10 \mathrm{ng} / \mathrm{ml}$ FGF2 and $10 \mu \mathrm{M}$ rock-inhibitor was added.

Conditioned MEF medium (CM-medium):

- DMEM

- $10 \%$ FBS

- $1 \%$ NEAA

- $1 \%$ Antibiotic Antimycotic

- $1 \%$ GlutaMax $^{\mathrm{TM}}$

supplemented with:

- $50 \mu \mathrm{g} / \mathrm{ml}$ ascorbic acid

- $10 \mathrm{ng} / \mathrm{ml}$ FGF2

- $10 \mu \mathrm{M}$ rock-inhibitor

MEF medium was added to confluent MEF culture and harvested after $24 \mathrm{~h}$. After sterile filtration CM was stored at -20 .

The outer rout sheet can be kept up to $48 \mathrm{~h}$ in DMEM at room temperature (RT) without keratinocytes losing viability.

\subsubsection{Culture of human Keratinocytes}

After the initial outgrowth, keratinocytes were cultured in serum-free and low calcium Epilife medium with HKGS supplement and addition of $10 \mu \mathrm{M}$ rockinhibitor Y-27632. Furthermore keratinocytes were transferred from matrigel coated T75 flasks to collagen IV coated 6-well-plates. Keratinocytes at $80 \%$ confluence were lifted via k-dispase digestion for $20 \mathrm{~min}$ at $37^{\circ} \mathrm{C}$ and plated on 6 well-plates which had been coated with collagen IV (1:100) for $1 \mathrm{~h}$ at $37^{\circ} \mathrm{C}$.To ensure a high reprogramming efficiency keratinocytes should not be passaged more than 2 passages. After the initial transfer from matrigel coated to collagen coated dishes TrypLE Express was used instead of dispase.

Keratinocytes Growth Medium:

- Epilife and HKGS supplement

- $10 \mu \mathrm{M}$ rock-inhibitor Y-27632 


\subsubsection{Freezing and Thawing of human Keratinocytes}

For cryopreservation keratinocytes were detached by TrypLE Express or dispase treatment, collected in a $15 \mathrm{ml}$ falcon and centrifuged at $1500 \mathrm{rpm}$ for $10 \mathrm{~min}$. After centrifugation cells were washed once with PBS - - and resuspended in Synt-aFreeze Medium. $1 \mathrm{ml}$ aliquots were pipetted in freezer-tubes and stepwise cooled to $-70^{\circ} \mathrm{C}$ in a cyro container (Nalgene, Neerijse, B) and stored in fluid nitrogen at $196^{\circ} \mathrm{C}$.

Aliquots of frozen keratinocytes were quickly thawed using a $37^{\circ} \mathrm{C}$ warm water bad and given into a $15 \mathrm{ml}$ falcon with $5 \mathrm{ml}$ Epilife. After centrifugation cells were seeded on collagen IV coated dishes in keratinocytes growth medium and cultured at $5 \% \mathrm{CO}_{2}$ and $95 \% \mathrm{O}_{2}$.

\subsubsection{Reprogramming of human Keratinocytes}

For infection, up to $3 \times 10^{5}$ keratinocytes per well of a 6 -well-plate were infected with $5 \times 10^{7}$ proviral genome copies (50 $\mu$ of the viral stock) in EpiLife medium supplemented with $8 \mu \mathrm{g} / \mathrm{ml}$ polyprene (Sigma-Aldrich) on two subsequent days. The viral construct which was used for reprogramming is described in 2.3.1.

At first, old medium was removed and cells were washed. Afterwards $1 \mathrm{ml}$ viral infection medium was added and a $50 \mu \mathrm{l}$ viral stock solution was carefully dropped to the cells. On the third day keratinocytes were detached with TrypLE Express (10 min at $37^{\circ} \mathrm{C}$ ) and split on irradiated REF feeder cells at the appropriate rate 1:3 [108]. Afterwards cells were cultured in hiPSCs medium in a $5 \% \mathrm{O}_{2}$ incubator and medium was changed daily.

Viral Infection Medium:

- Epilife

- $8 \mu \mathrm{g} / \mathrm{ml}$ polyprene

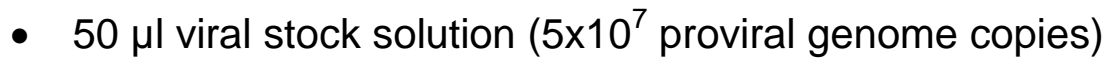

HiPSCs Medium:

- Knockout DMEM

- $20 \%$ Knockout Serum Replacement

- $1 \%$ NEAA

- $1 \%$ Antibiotic Antimycotic 
- $1 \%$ GlutaMax $^{\mathrm{TM}}$

- $100 \mu \mathrm{M} \beta$-mercaptoethanol

supplemented with:

- $50 \mu \mathrm{g} / \mathrm{ml}$ ascorbic acid

- $10 \mathrm{ng} / \mathrm{ml}$ FGF2

- $10 \mu \mathrm{M}$ rock-inhibitor

\subsubsection{Culture of MEFs and REFs}

\subsubsection{Preparation and culturing of MEFs and REFS}

CF-1 MEFs from embryonic day E12.5 or E14.5 (Stemcell Technologies, Vancouver, CA, USA) were acquired by purchase.

REFs were isolated from day E14 Sprague Dawley rat embryos as described earlier for mice [192]. The pregnant rat was killed with an overdose of CO2. The uterus was taken out and the embryos were freed from the egg integuments and laid into a pre cooled dissection solution. Under a microscope, cooled in dissection solution, the embryos were decapitated. Then the limbs and the liver were removed and the remaining body minced into small pieces and collected in dissection solution. After centrifugation at $1500 \mathrm{rpm}$ for $2 \mathrm{~min}$, the pellet was washed twice with PBS - - and dissociated using $10 \mathrm{ml}$ of $0.125 \%$ Trypsin-Versen solution and glass beads for $30 \mathrm{~min}$ in a $37^{\circ} \mathrm{C}$ water bad. After $30 \mathrm{~min}$ the cell suspension was filtered through a sterile nylon mesh (100-200 nm) to completely disperse any remaining tissue. After filtration cells were again centrifuged at 1500 rpm for 2 min and then resuspended in REF medium and cultured in T175 flasks coated with $0.1 \%$ gelatine at $37^{\circ} \mathrm{C}$ with $5 \% \mathrm{CO} 2$ and $5 \% \mathrm{O} 2$.

REF / MEF medium:

- DMEM

- $10 \%$ FBS

- $1 \%$ NEAA

- $1 \%$ Antibiotic Antimycotic

- $1 \%$ GlutaMax $^{\mathrm{TM}}$

supplemented with:

- $50 \mu \mathrm{g} / \mathrm{ml}$ ascorbic acid 
Dissection Solution:

- $1 \%$ Antibiotic Antimycotic

- $1 \%$ D-Glucose (Invitrogen)

- 200 ml HBSS - -

Trypsin $0.125 \%$ Versen

- $5 \%$ Trypsin $0.25 \%$ (Biochrom AG, Berlin)

- $45 \%$ PBS - - (Invitrogen)

- $50 \%$ Versen

Versen

- $8 \mathrm{~g} \mathrm{NaCl}$

- $0.2 \mathrm{~g} \mathrm{KCL}$

- $1.382 \mathrm{~g} \mathrm{Na}_{2} \mathrm{HPO}_{4}$

- $0.29 \mathrm{~g} \mathrm{KH}_{2} \mathrm{PO}_{4}$

- $0.5 \mathrm{~g}$ EDTA

$\rightarrow$ ad $1 \mathrm{l} \mathrm{H}_{2} \mathrm{O}$ bidest, autoclave

Cells were passaged by $0.125 \%$ Trypsin-Versen digestion when reaching confluence for up to 5 passages. For passaging, the old medium was removed and the cells were washed with PBS - - . Then, $500 \mu \mathrm{l}$ of $0.125 \%$ Trypsin-Versen solution was added to each well and incubated for $10 \mathrm{~min}$ at $37^{\circ} \mathrm{C}$. After incubation, $2 \mathrm{ml}$ PBS - - were added to each well to inhibit trypsin activity. Cells were collected in a $15 \mathrm{ml}$ tube and centrifuged at $1500 \mathrm{rpm}$ for $2 \mathrm{~min}$. The pellet was resuspended in MEF / REF medium and cells were plated onto $0.1 \%$ gelatin coated T175 flasks at the appropriate rate 1:5.

\subsubsection{Freezing and Thawing of MEFs / REFs}

For cryopreservation cells were detached by $0.125 \%$ Trypsin-Versen digestion, centrifuged at $1500 \mathrm{rpm}$ for $2 \mathrm{~min}$ and resupended in fibroblast freezing medium. $1 \mathrm{ml}$ aliquots were pipetted in freezer tubes and stepwise cooled to $-70^{\circ} \mathrm{C}$ in a cyro container and stored in fluid nitrogen at $-196^{\circ} \mathrm{C}$.

Aliquots of frozen MEFs / REFs were quickly thawed using a $37^{\circ} \mathrm{C}$ warm water bad and given into a $15 \mathrm{ml}$ falcon with $5 \mathrm{ml} \mathrm{MEF/REF} \mathrm{medium.} \mathrm{Cells} \mathrm{were}$ centrifuges at $1500 \mathrm{rpm}$ for $2 \mathrm{~min}$ and immediately resuspended in MEF /REF 
medium containing Vit.C. Cells were placed on $0.1 \%$ gelatin coated T175 flasks and cultured at $5 \% \mathrm{CO}_{2}$ and $5 \% \mathrm{O}_{2}$.

Fibroblast Freezing Medium:

- $10 \%$ DMSO

- $40 \%$ MEF / REF medium

- $50 \%$ FBS

\subsubsection{Irradiation of MEFs / REFS}

Cells were detached as described above, collected in a $50 \mathrm{ml}$ tube, centrifuged and resuspended in MEF / REF medium. Cells were counted with an Neubauer counting chamber (Brandt, Wertheim, BW, Germany) to obtain a density of $3 \times 10^{5}$ cells per well of a 6-well-plate. Subsequently, the appropriate amount of cells was irradiated with $30 \mathrm{~Gy}$ in suspension. Afterwards irradiated feeder cells were plated onto $0.1 \%$ gelantine-coated wells in MEF / REF medium. Usually MEFs / REFs have been irradiated one day before use.

\subsubsection{Culture of HEK T293 (Lenti-X) cells}

Lenti-X cells were used for lentiviral production. Cells were cultured in Lenti-X medium in $94 \mathrm{~mm}$ Petri dishes (Nunc, Wiesbaden, HV, Germany). Lenti-X cells were passaged every 2-4 days. For passaging, the old medium was displaced with $5 \mathrm{ml}$ fresh Lenti-X medium. Cells were gently detached by blowing off using a $5 \mathrm{ml}$ pipette and collected in a $15 \mathrm{ml}$ tube. Then, Lenti-X cells were centrifuged at 1500 rpm for 2 min and seeded on $94 \mathrm{~mm}$ Petri dishes with the rate 1:5 (up to 1:10) and cultured in $5 \% \mathrm{CO}_{2}$ and $95 \% \mathrm{O}_{2}$. Freezing and thawing procedure was performed as described for MEFs / REFs.

Lenti-X medium:

- DMEM

- $15 \%$ FBS

- $1 \%$ NEAA

- $1 \%$ Antibiotic Antimycotic

- $1 \%$ GlutaMax $^{\mathrm{TM}}$ 


\subsubsection{Culture of human induced pluripotent Stem Cells}

\subsubsection{Culture of hiPSCs on Feeder Cells}

After viral transduction, keratinocytes were detached with TrypLE Express digestion and transferred to irradiated REF feeder cells at the rate 1:3. Human iPSCs were cultured in hiPSCs medium at $37^{\circ} \mathrm{C}$ with $5 \% \mathrm{CO} 2$ and $5 \% \mathrm{O} 2$. Medium was changed daily $(1.5 \mathrm{ml}$ per well). Colonies showing a typical hiPSCs like morphology emerged around 3-5 days later. After reaching the appropriate size, hiPSCs were mechanically passaged on to MEF feeder cells. From passage 2 on hiPSCs can usually be passaged via hESC-dispase digestion (described in 2.2.3.2.). From passage 3 on hiPSCs were cultured under feeder-free conditions.

\subsubsection{Culture of hiPSCs under feeder-free and serum-free Conditions}

Human iPS cells were cultured in mTeSR1 medium on hESC-qualified-matrigel coated wells of a 6-well-plate. For coating $500 \mu \mathrm{l} /$ well were incubated for $1 \mathrm{~h}$ at room temperature. Usually medium of hiPSC cultures was changed daily and hiPSCs were splitted as soon as colonies began to fuse. For passaging, hiPSCs were detached by hESC-dispase digestion ( $1 \mathrm{mg} / \mathrm{ml}$ ). After removing the old medium, cells were washed with DMEM/F12 and $500 \mu \mathrm{l}$ hESC-dispase were added to each well and incubated for $5-7 \mathrm{~min}$ at $37^{\circ} \mathrm{C}$. HESC-dispase was aspirated from the cells when the edge began to curl. After an additional washing step, $1 \mathrm{ml}$ of hiPSCs medium was added to each well and hiPSC colonies were carefully detached using a cell scraper (TPP, Switzerland), collected in a $50 \mathrm{ml}$ tube and immediately transferred to either MEF feeder cells or to matrigel coated dishes. HiPSC colonies should not be dissociated into a single cell suspension, as cell survival is dramatically reduced.

HiPSCs were kept in culture up to 40 passages. Differentiated cells were mechanically scratched out every day.

\subsubsection{Freezing and Thawing of hiPSCs}

Human stem cells colonies were detached via dispase digestion and centrifuged at $300 \mathrm{~g}$ for $5 \mathrm{~min}$. Afterwards cells were resuspended in $\mathrm{mFreSR}$ medium and $1 \mathrm{ml}$ aliquots were pipetted in freezer tubes and stepwise cooled to $-70^{\circ} \mathrm{C}$ in a cyro container and stored in fluid nitrogen at $-196^{\circ} \mathrm{C}$. 
Before thawing hiPSCs, MEF-feeder cells or matrigel coated dishes should be prepared. Frozen aliquots were thawed quickly using a $37^{\circ} \mathrm{C}$ water bad. HiPSCs were resuspended in mTeSR 1 or hiPSCs medium and centrifuged at $300 \mathrm{~g}$ for 5 min. Afterwards cells were seeded on MEFs or matrigel coated dishes.

\subsubsection{Generation of clonal hiPSCs Lines}

For generation of a clonal hiPSCs line, one single hiPSC colony, which had been developed after reprogramming, was mechanically scraped out using a sterile scalpel and transferred to MEF feeder cells. As described above clonal hiPSCs were cultured under feeder-free conditions in mTeSR1 from passage 3 on. During the first passages clonal colony number had been relatively little, so attachment of hiPSCs had to be improved during splitting procedure. Using a mixture of $50 \%$ mTeSR 1 medium and $50 \% \mathrm{CM}$-medium for passaging procedure resulted in an enhanced survival of hiPSCs colonies. After $24 \mathrm{~h}$ of incubation medium was again changed to mTeSR1.

\subsubsection{Generation of virus-free hiPSCs lines via CRE-treatment}

The lentiviral stem cell cassette vector, which had been used in this study, is flanked by loxP sites for CRE-mediated excision and generation of virus-free hiPSC lines. Human hiPSCs were maintained on matrigel coated wells of a 6-wellplate in mTeSR1 medium. One hour before passaging, $10 \mu \mathrm{M}$ rock-inhibitor was added. This step is crucial for preventing apoptosis of hiPSCs in single cell suspension. Next, $500 \mu \mathrm{l}$ / well alfazyme were added and incubated for $20-30$ $\min$ at $37^{\circ} \mathrm{C}$. Cells were then harvested and dissociated into a single cell suspension. After counting, 50.000-100.000 hiPSCs per well of a 6-well-plate precoated with matrigel were seeded and cultured in mTeSR1 supplemented with $10 \mu \mathrm{M}$ rock-inhibitor.

Before CRE-protein treatment hiPSCs need time to attach. Ideally, CRE-protein treatment should be started $24 \mathrm{~h}$ after passaging. After removing the old medium, $1.5 \mathrm{ml} \mathrm{mTeSR} 1$ containing up to $1.5-2 \mu \mathrm{M}$ of TAT-CRE protein was added to each well. Higher concentrations will result in protein precipitation and cell death. After 5 hours, medium was changed to $50 \%$ mTeSR 1 and $50 \% \mathrm{CM}$ medium for $24 \mathrm{~h}$. At the next day medium was changed to mTeSR1. Genotype was checked by PCR analysis. 
CRE-medium had been prepared by diluting TAT-CRE protein from the glycerol stock with mTeSR1 medium. Afterwards CRE-medium had been filtered through a sterile low protein-binding filter $(0.2 \mu \mathrm{m})$ into falcon tubes. Efficiency of CREtreatment depended on TAT-CRE batch: $50-100 \%$.

CRE medium:

- mTeSR1

- $1.5-2 \mu \mathrm{M}$ TAT-CRE protein

- sterile filtered

\subsubsection{Genotyping of virus-free hiPSCs by PCR Analysis}

\subsection{Purification of hiPSCs DNA}

First of all, hiPSCs cells (one well of a 6 -well plate) were dissociated by $0.125 \%$ Trypsin-Versen digestion. After removing the old medium and washing with PBS - , $500 \mu \mathrm{l} 0.125 \%$ Trypsin-Versen were added to the well and incubated for $10 \mathrm{~min}$ at $37^{\circ} \mathrm{C}$. Cells were then dissociated into a single-cell suspension, collected and centrifuged at $1500 \mathrm{rpm}$ for $3 \mathrm{~min}$. After centrifugation cells were resuspended in $200 \mu \mathrm{l}$ PBS - -. DNA purification was assessed using QIAamp DNA Mini Kit (Qiagen).

$20 \mu \mathrm{l}$ protease $\mathrm{K}$ (Qiagen) were pipetted into the bottom of a $1.5 \mathrm{ml}$ microcentrifuge tube and $200 \mu \mathrm{l}$ hiPSCs suspension were added to the microcentrifuge tube. Then $200 \mu \mathrm{l}$ Buffer AL were added and suspension was mixed by pulse-vortexing for $15 \mathrm{~s}$. In order to ensure efficient lyses, it is essential that the sample and Buffer $\mathrm{AL}$ are thoroughly mixed to yield a homogenous solution. Cells suspension was incubated at $56^{\circ} \mathrm{C}$ for $10 \mathrm{~min}$ and briefly centrifuged to remove drops from the inside of the lid. $200 \mu \mathrm{l} 100 \%$ ethanol were added followed by pulse-vortexing for $15 \mathrm{~s}$. After mixing, tube was briefly centrifuged. The mixture was then applied to the QIAamp Mini spin column (in a 2 $\mathrm{ml}$ collection tube) without wetting the rim and centrifuged at $8000 \mathrm{rpm}$ for $1 \mathrm{~min}$. Afterwards the QIAamp Mini spin column was placed in a new collection tube. Now, $500 \mu \mathrm{l}$ Buffer AW1 were added to the QIAamp Mini spin column and centrifuged at $8000 \mathrm{rpm}$ for $1 \mathrm{~min}$. After centrifugation, the QIAamp Mini spin column was placed in a new collection tube. $500 \mu \mathrm{l}$ Buffer AW2 were added and QIAamp Mini spin column was centrifuged at full speed (14,000 rpm) for 3 min. 
Centrifugation was repeated with a new collection tube. QIAamp Mini spin column was placed in a $1.5 \mathrm{ml}$ microcentrifuge tube and $200 \mu \mathrm{l}$ destilled water were carefully added and incubated for $5 \mathrm{~min}$ at room temperature. After incubation tube was centrifuged at $8000 \mathrm{rpm}$ for $1 \mathrm{~min}$. The obtained DNA can be stored at $-80^{\circ} \mathrm{C}$.

QIAamp Mini Kit (250):

- QIAamp Mini Spin Columns

- Collection Tube (2ml)

- Proteinase K

- Buffer AL

- Buffer ALT

- Buffer (AW1) concentrate

- Buffer AE

\subsection{Polymerase chain reaction}

DNA fragments were selectively amplified, utilizing the polymerase chain reaction (PCR). As template DNA of both CRE-treated and non-treated cells was used. Primers bind at $5^{\prime}$ respectively $3^{`}$ end of the DNA template. Primer binding is followed by amplification of the DNA template by thermo stable Taq-polymerase. For PCR goTaq Green Master Mix (Promega) was used. Besides the TaqPolymerase it contains a Pfu-polymerase with proofreading activity, a dNTP mix and the required buffer.

Table 3: PCR Primers

\begin{tabular}{|c|c|c|}
\hline Primer for PCR & 5`-sequence- $3^{`}$ & size of PCR product \\
\hline $\begin{array}{c}\text { STEMCCA } \\
\text { Primer-forward }\end{array}$ & CAGGCTGTGGCAAAACCTAT & $400 \mathrm{bp}$ \\
\hline $\begin{array}{c}\text { STEMCCA } \\
\text { Primer-reverse }\end{array}$ & CCCTAGGAATGCTCGTCAAG & \\
\hline $\begin{array}{c}\text { Actin } \\
\text { Primer-forward }\end{array}$ & GGCTACAGCTTCACCACCAC & $480 \mathrm{bp}$ \\
\hline $\begin{array}{c}\text { Actin } \\
\text { Primer-reverse }\end{array}$ & CCACATCTGCTGGAAGGTGG & \\
\hline
\end{tabular}

Primer-mixture:

$10 \mu \mathrm{l}$ forward-primer $+10 \mu \mathrm{l}$ reverse primer 
PCR-mixture:

- $12.5 \mu \mathrm{l}$ GoTaq Green Master Mix

- $1 \mu \mathrm{l}$ Primer-mixture

- $11.5 \mu \mathrm{l}$ DNA (> $5 \mathrm{ng})$

The PCR was set up in a thermocycler (Eppendorf, Hamburg, Germany) with the following program:

\begin{tabular}{|c|c|c|c|}
\hline Step & Temperature & Duration & Cycles \\
\hline Denaturation & $95^{\circ} \mathrm{C}$ & $2 \mathrm{~min}$ & 1 \\
\hline Denaturation & $95^{\circ} \mathrm{C}$ & $30 \mathrm{~s}$ & 35 \\
\hline Primer attachment & $55^{\circ} \mathrm{C}$ & $30 \mathrm{~s}$ & 35 \\
\hline Elongation & $72^{\circ} \mathrm{C}$ & $30 \mathrm{~s}$ & 35 \\
\hline Elongation & $72^{\circ} \mathrm{C}$ & $5 \mathrm{~min}$ & 1 \\
\hline End & $4^{\circ} \mathrm{C}$ & & \\
\hline
\end{tabular}

\subsection{DNA agarose gel electrophoresis}

DNA as a negatively charged molecule can be moved through a matrix of agarose by electric current and be separated by size. This is how DNA fragments can easily be analyzed after PCR amplification.

$2 \%$ agarose was boiled up in $1 \times$ TAE buffer and cooled down to $60{ }^{\circ} \mathrm{C}$. After adding ethidiumbromide (Roth, Karlsruhe, BW, Germany) in a concentration of 0.5 $\mu \mathrm{g} / \mathrm{ml}$, the solution was then poured into a gel chamber for 15-20 min. In the meantime, DNA samples were mixed with 6x loading dye solution (Fermentas, St. Leon-Rot, BW, Germany). Subsequently, the gel electrophoresis was performed and a constant voltage of $85 \mathrm{~V}$ was applied. The gel was finally analyzed by UV light photography.

\subsubsection{Differentiation and Culture of Motoneurons derived from hiPSCs}

\subsubsection{Differentiation of human iPSCs into motoneurons}

Differentiation of human iPSCs into motoneurons was modified after [82].

Differentiation into motoneurons can be divided into 4 steps related to ongoing differentiation and change of transcription factor expression. 
Step 1 day 1-4: Generation of embryoid Bodies (EBs)

HiPSCs were grown at hESC-MG coated 6-well plates in mTeSR1 medium. Differentiation protocol was usually performed with 2 confluent 6-well plates. $\mathrm{DMEM} / \mathrm{F} 12$ and $\mathrm{hESC}$-dispase was warmed to $37^{\circ} \mathrm{C}$. The old medium was removed from the cells and cells were rinsed with DMEM/F12. $500 \mu \mathrm{hESC}$ dispase were add to each well and incubated for $5-7 \mathrm{~min}$ at $37^{\circ} \mathrm{C}$. Dispase was aspirated from the cells when the edges began to curl. Cells were gently washed with DMEM/F12 and $1 \mathrm{ml}$ of EB medium was added to each well. Colonies were mechanically lifted using a cell scraper and collected in a $50 \mathrm{ml}$ tube. Cells were then transferred to two T75 low-attachment flasks (Corning, Corning, NY, USA) and filled up with EB-medium up to $10 \mathrm{ml}$ per flask. Addition of rock-inhibitor Y27632 for the first $48 \mathrm{~h}$ improved survival of hiPSCs and promoted generation of EBs as well. Medium was changed daily. For medium change, the flasks were allowed to stand so that the aggregates sink for $5 \mathrm{~min}$. The hiPSCs colonies generally round up as spheres with some individual cell debris floating in the culture. Debris attaching to the spheres was stripped off by pipetting the culture with a $5 \mathrm{ml}$ pipette. After $5 \mathrm{~min}$ the spheres usually sank to the bottom and $7 \mathrm{ml}$ of the old medium including was carefully removed and replaced with $7 \mathrm{ml}$ fresh EB medium.

Step 2 day 4-15: Generation of neuroepithelial Rosettes (NE)

At day 4 medium was changed to differentiation medium 1. Neural growth factors like BDNF, GDNF, IGF1 promote neural differentiation. On day 7 spheres were collected into a $50 \mathrm{ml}$ tube and centrifuged at $50 \mathrm{~g}$ for $3 \mathrm{~min}$. Before seeding the spheres, 6 -well-plates had been coating with $400 \mu$ laminin (1:25) per well ( $1 \mathrm{~h}$ at $37^{\circ} \mathrm{C}$ ). Spheres were seeded in $1 \mathrm{ml}$ differentiation medium 1 and were cultured in $5 \% \mathrm{CO}_{2}$.

On the following day medium was filled up to $2 \mathrm{ml}$. Medium had to be changed three times a week. On day 10 emerging neural rosettes were evaluated under the microscope. The old medium was removed and replaced with differentiation medium 2 containing $0.1 \mu \mathrm{M} R A$ to stimulate posteriorization of the cells. 
Step 3 day 15-28: Generation of Motoneuron Progenitors (pMNs)

After one week a high amount of neural tube-like cells were visible under the microscope. Cells which did not possess typical rosettes were mechanically taken out. The old medium was removed, cells were washed once with DMEM/F12 and $2 \mathrm{ml}$ of differentiation medium 3 were added to each well. NE cells were loosely attached to the substrate whereas non-neural cells were much more fixated to the Petri dish. Based on this, NE cells were gently detached by rinsing off using a $5 \mathrm{ml}$ pipette and collected in a conical $50 \mathrm{ml}$ tube. This procedure was repeated once to ensure that all neuroepithelial cells had been successfully removed. If there had been very huge spheres, cells were carefully pipetted with a $10 \mathrm{ml}$ pipette to be dissociated into smaller spheres, but not to a single cells suspension or smaller than $100 \mu \mathrm{m}$. Cells were centrifuged at $50 \mathrm{~g}$ for $2 \mathrm{~min}$ and transferred to T75 lowattachment flasks. Purmorphamine, a SHH agonist leads to caudalization of the cells whereas B27 promotes neural cells survival and proliferation. At this stage, pMNs can be cultured for up to 3 months. Motoneuron progenitors still proliferate so it is possible to increase cell amount by passaging (described in 2.2.4.3.). Medium was changes three times a week.

\section{Step 4 day 28 - X: Generation of postmitotic Motoneurons}

Motoneuron progenitors were seeded either on glass coverslips or on treated ibidis (35 mm high, tissue culture treated, Ibidi, Munic, BY, Germany) for immunocytochemistry. Cells which were planned to be harvested for mRNA analysis were cultured in 24-well plates. In both cases dishes were pre-coated with Poly-L-Ornithin (PLO) for $1 \mathrm{~h}$ at $37^{\circ} \mathrm{C}$ or over night at $4^{\circ} \mathrm{C}$. After removing PLO, dishes were washed three times with PBS - - and then coated with laminin (1:25) for $1 \mathrm{~h}$ at $37^{\circ} \mathrm{C}$. For long term culture ( $>4$ weeks) treated ibidis approved highest cell survival. Motoneuron progenitors were collected in a $15 \mathrm{ml}$ tube and centrifuged at $50 \mathrm{~g}$ for $3 \mathrm{~min}$. Afterwards the pellet was carefully resuspended with differentiation medium 4 and cells were seeded at prepared dishes. When cultured in ibidis $2 \mathrm{ml}$ of medium was needed and when cultured in 24-well plated $500 \mu \mathrm{l}$ medium per well was used. Half amount of medium was replaced with differentiation medium 4 three times a week. In differentiation medium 4 the concentration of RA and purmorphamine was reduced otherwise terminal differentiation to a postmitotic fate would be inhibited. In long term culture and 
especially before electrophysiological measurements $0.2 \%$ FBS was added once a week.

EB medium:

- DMEM/F12

- $20 \%$ knockout Serum Replacement

- $1 \%$ NEAA

- $1 \%$ Antibiotic Antimycotic

- $1 \%$ GlutaMax ${ }^{\mathrm{TM}}$

- $100 \mu \mathrm{M} \beta$-mercaptoethanol

supplemented with (for the first $48 \mathrm{~h}$ ):

- $10 \mu \mathrm{M}$ rock-inhibitor

Basic differentiation medium:

- $\mathrm{DMEM} / \mathrm{F} 12$

- $1 \%$ NEAA

- $1 \%$ Antibiotic Antimycotic

- $2 \mu \mathrm{g} / \mathrm{ml}$ heparin

- $2 \% \mathrm{HM}$

Hormone Mix (HM), 100ml:

- $20 \mu \mathrm{g} / \mathrm{ml}$ insulin:

$\rightarrow 100 \mathrm{mg}$ Insulin were dissolved in $0.1 \mathrm{M} \mathrm{HCL}, 36 \mathrm{ml}$ distilled $\mathrm{H}_{2} \mathrm{O}$ were added and mixed.

- $7.72 \mu \mathrm{g} / \mathrm{ml}$ putrescin (Sigma-Aldrich):

$\rightarrow 38.6 \mathrm{ml}$ putrescin were dissolved in $40 \mathrm{ml}$ distilled $\mathrm{H}_{2} \mathrm{O}$ and added to the insulin-solution.

$\rightarrow$ add $40 \mu \mathrm{l}$ of sodium selenite stock solution

$\rightarrow$ add $40 \mu$ of progesterone stock solution

$\rightarrow$ add $400 \mathrm{mg}$ apotransferrin (Serologicals, Atlanta, GA, USA)

$\rightarrow$ mixture was filled up with distilled $\mathrm{H}_{2} \mathrm{O}$ up to $200 \mathrm{ml}$

$\rightarrow$ aliquot and store at $-20^{\circ} \mathrm{C}$ 
Differentiation medium 1 day 4 - 10:

- Basic differentiation medium

supplemented with:

- $10 \mathrm{ng} / \mathrm{ml}$ GDNF

- $10 \mathrm{ng} / \mathrm{ml}$ BDNF

- $10 \mathrm{ng} / \mathrm{ml}$ IGF1

- $0.1 \mu \mathrm{M}$ cAMP

- $20 \mu \mathrm{g} / \mathrm{ml}$ Ascorbic Acid

Differentiation medium 2 day 10 - 15:

- Differentiation medium 1

supplemented with:

- $0.1 \mu \mathrm{M} R A$

Differentiation medium 3 day 15 - 28:

- Differentiation medium 1

supplemented with:

- $0.1 \mu \mathrm{M} R A$

- $1 \mu \mathrm{M}$ Purmorphamine

- $2 \%$ B27 without Vit. A

Differentiation medium 4 day $28-x x$ :

- Differentiation medium 1

supplemented with:

- $0.05 \mu \mathrm{M}$ RA

- $0.5 \mu \mathrm{M}$ Purmorphamine

- $2 \%$ B27 without Vit. A

\subsubsection{Passaging of hiPSCs derived Motoneurons}

Motoneurons derived from hiPSCs were splitted during motoneuron progenitor state. Big clusters were collected in a 15- or $50 \mathrm{ml}$ conical tube and centrifuged at $50 \mathrm{~g}$ for $2 \mathrm{~min}$. The supernatant was carefully removed and cells were resuspended in $800 \mu \mathrm{l}$ StemPro-Accutase. The clusters were incubated for $4 \mathrm{~min}$ 
at $37^{\circ} \mathrm{C}$. During incubation, a Pasteur pipette was fire polished to narrow down the diameter. Before using, the pipette was briefly rinsed with DMEM/F12 to prevent cells clusters from sticking onto the pipette. After incubation cells were dissociated with the prepared Pasteur pipette and $2 \mathrm{ml}$ medium was added to the cells. Cells were not dissociated to single cell suspension. Then, dissociated cells were centrifuged at $50 \mathrm{~g}$ for $2 \mathrm{~min}$. Subsequently, the supernatant was decanted and the pellet was resuspended in differentiation medium 3 and then transferred to T75 low-attachment flasks filled with $10 \mathrm{ml}$ of differentiation medium 3. To promote cell reaggregation and proliferation LIF (1:100) was added to the medium for $24 \mathrm{~h}$ subsequently after dissociation.

\subsubsection{Preparation and Culture of primary Motoneurons}

Our protocol was modified after Martinou et al [129]. Pregnant rats were killed with an overdose of $\mathrm{CO}_{2}$. Embryos were taken out of the uterus and freed from egg integuments and transferred to a pre-cooled dissection solution. All preparation steps were performed on ice. Under a microscope the embryos were decapitated and several layers of embryonic tissues were removed to expose the spinal cord. The spinal cords were carefully dissected and freed from meninges and dorsal root ganglia. The spinal cords were minced into pieces and collected in a $15 \mathrm{ml}$ tube containing dissection solution. Afterwards, the falcons containing the spinal cords were centrifuges at $1500 \mathrm{rpm}$ for $2 \mathrm{~min}$ at room temperature. The pellet was digested with TrypLE Express for 15 min at RT. Incubation was stopped by filling up the falcon with $10 \mathrm{ml}$ PBS - - , followed by centrifugation at $1500 \mathrm{rpm}$ for $2 \mathrm{~min}$. Further dissociation was performed by adding $400 \mu \mathrm{l}$ of DNAse I (diluted as described above) for $10 \mathrm{~min}$ at $37^{\circ} \mathrm{C}$. Again reaction was stopped by adding PBS - and the cells were centrifuged at $1500 \mathrm{rpm}$ for $2 \mathrm{~min}$. Pellet was resuspended in $3 \mathrm{ml}$ primary motoneuron medium (PMN medium) and dissociated with a firepolished (described above) Pasteur pipette into a single cell suspension. Single cells suspension was layered onto a $3.5 \%$ BSA solution and then centrifuged at $80 \mathrm{~g}$ for $20 \mathrm{~min}$ at RT. After centrifugation cells were resuspended in PBS - - and carefully layered onto the discontinuous Nycodenz-gradient. The gradient was centrifuged at $700 \mathrm{~g}$ for $20 \mathrm{~min}$ at $4^{\circ} \mathrm{C}$. Following, the upper $3 \mathrm{ml}$ of the gradient were collected and $9 \mathrm{ml}$ primary motoneuron medium (PrMN medium) was added. The cell suspension was again centrifuged at $1000 \mathrm{~g}$ for $10 \mathrm{~min}$ at $4^{\circ} \mathrm{C}$. After 
centrifugation the supernatant was discarded and the pellet was resuspended in PrMN medium containing $2 \%$ knockout serum replacement. The cells were counted with a Neubauer counting chamber and $18 \times 10^{4}$ cells were seeded per well of a 24-well plate. Primary motoneurons were plated either on $13 \mathrm{~mm}$ glass coverslips for immunocytochemistry. Cells which were planned to be harvested for mRNA analysis were cultured in 24-well plates. In both cases dishes were precoated with PLO for $1 \mathrm{~h}$ at $37^{\circ} \mathrm{C}$ or over night at $4^{\circ} \mathrm{C}$. After removing PLO, dishes were washed three times with PBS - - and then coated with laminin (1:50, 10 $\mu \mathrm{g} / \mathrm{ml}$ ) for $1 \mathrm{~h}$ at $37^{\circ} \mathrm{C}$. Cells were incubated at $5 \% \mathrm{CO}_{2}$ and $95 \% \mathrm{O}_{2}$. After 3 hours, the medium was changed to PrMN medium without knockout serum replacement.

Dissection solution:

- HBS - -

- $1 \%$ Antibiotic Antimycotic

- $1 \%$ D-Glucose (Invitrogen)

$3.5 \%$ BSA (bovine serum albumin) solution:

- $10 \mathrm{ml}$ PBS - -

- 0.35 g BSA (RIA Grade, Sigma Aldrich)

Primary motoneuron medium (PrMN medium):

- $33 \%$ DMEM

- $66 \%$ knockout DMEM F12

- $1 \%$ NEAA

- $1 \%$ Antibiotic Antimycotic

- $1 \%$ GlutaMax ${ }^{\mathrm{TM}}$

- $2 \%$ B27 without Vit.A

supplemented with (for the first 2 h):

- $1 \%$ knockout Serum Replacement 
Nycodenz-gradient:

A $30 \%$ solution of Nycodenz was prepared by mixing $1.5 \mathrm{ml}$ Nycodenz $60 \%$ with $1.5 \mathrm{ml}$ PBS - -

Three different solutions of increasing density were prepared:

\begin{tabular}{|c|c|c|}
\hline Solution & Nycodenz $30 \%$ & PBS - - \\
\hline $\mathbf{1}$ & $750 \mu \mathrm{l}$ & $2250 \mu \mathrm{l}$ \\
\hline $\mathbf{2}$ & $1000 \mu \mathrm{l}$ & $2000 \mu \mathrm{l}$ \\
\hline $\mathbf{3}$ & $1250 \mu \mathrm{l}$ & $1750 \mu \mathrm{l}$ \\
\hline
\end{tabular}

The solution 1 was given into a $15 \mathrm{ml}$ falcon. Solution 2 was carefully given beneath solution 1 using $5 \mathrm{ml}$ syringe. At last, solution 3 was added beneath solution 2. Usually five Nycodenz-gradients had to be prepared.

\subsubsection{Preparation and Culture of primary Myotubes}

\subsubsection{Preparation of primary Myotubes}

Our protocol was modified after [8]. Before beginning, 6-well-plates were coated with PLO for $1 \mathrm{~h}$ at $37^{\circ} \mathrm{C}$, washed three times with PBS - - and subsequently coated with laminin $(1: 25,20 \mu \mathrm{g} / \mathrm{ml})$ for $1 \mathrm{~h}$ at $37^{\circ} \mathrm{C}$.

Neonate mice (1-4 day old) were briefly anesthetized, decapitated and immediately put in ice-cooled dissection solution (described in 2.2.5.). All preparation steps were performed on ice. Under a microscope the skin of the muscles was removed and muscle tissue was taken from $M$. quadriceps femoris, M. biceps femoris, M. triceps surae, M. biceps brachii and M. triceps brachii. Tissue samples were minced, harvested in a $15 \mathrm{ml}$ tube and centrifuged at $1000 \mathrm{~g}$ for $5 \mathrm{~min}$. Cells were resuspended in $2.5 \mathrm{ml} \mathrm{HBSS}$ - - and digested with $0.2 \%$ pronase solution for $1 \mathrm{~h}$ at $37^{\circ} \mathrm{C}$ under constant agitation. After digestion cells tissue samples were washed with HMBS - - and resuspended with $5 \mathrm{ml}$ of primary myotube medium (PMT medium). Subsequently, cells were isolated by triturating with a fire-polished Pasteur pipette into a single cell suspension. Cells were then filtered through a $70 \mu \mathrm{m}$ nylon filter and centrifuged at $1000 \mathrm{~g}$ for $5 \mathrm{~min}$. The pellet was resuspended in $1.5 \mathrm{ml}$ of PMT medium and layered onto the Percoll-gradient. The gradient was centrifuged with brake off at $1.250 \mathrm{~g}$ for $20 \mathrm{~min}$ at room temperature. After centrifugation cells were harvested from the 0-35\% and the 35 $\%-70 \%$ interface and collected in a $15 \mathrm{ml}$ tube. Cells were washed with HMBS - and resuspended in PMT medium and plated to the coated 6-well-plates. Primary 
myotubes were cultured in $5 \% \mathrm{CO}_{2}$ and $95 \% \mathrm{O}_{2}$. From passage 2 on, cells were cultured in primary myotube anti-differentiation medium.

\section{$0.2 \%$ Pronase solution:}

- $250 \mu \mathrm{HBS}$ - -

- $500 \mu \mathrm{l} 2 \%$ pronase

- $1.75 \mathrm{ml} \mathrm{H}_{2} \mathrm{O}$

- $2.5 \mathrm{ml}$ cell suspension in HBSS - -

Primary myotube medium (PMT medium):

- $50 \%$ DMEM 2x

- $28 \%$ destilled $\mathrm{H}_{2} \mathrm{O}$

- $15 \%$ Horse serum (heat inactivated)

- $3 \%$ Chick embryo extract

- $1 \%$ Antibiotic Antimycotic

- $1 \%$ GlutaMax $^{\mathrm{TM}}$

- $1 \%$ Sodium Pyruvate

- $1 \%$ HEPES-buffer

Percoll-gradient:

A $90 \%$ Percoll stock solution was prepared as described above. Two different solutions of increasing density were prepared:

\begin{tabular}{|c|c|c|}
\hline Solution & Percoll 90 \% & HMBSS - - \\
\hline $\mathbf{1}$ (Percoll 35\%) & $1.56 \mathrm{ml}$ & $2.44 \mathrm{ml}$ \\
\hline $\mathbf{2}$ (Percoll 70\%) & $3.1 \mathrm{ml}$ & $0.9 \mathrm{ml}$ \\
\hline
\end{tabular}

The solution 1 was given into a $15 \mathrm{ml}$ falcon. Solution 2 was carefully given beneath solution 1 using $5 \mathrm{ml}$ syringe.

\subsubsection{Passaging of Myoblasts}

Myoblasts were passaged when they had reached about $60 \%$ confluence. If cells became more than $60 \%$ confluent, differentiation into myotubes is induced. Myoblasts were detached via $0.125 \%$ Trypsin-Versen solution ( $3 \mathrm{~min}$ at $37^{\circ} \mathrm{C}$ ). After incubation cells were collected in a $15 \mathrm{ml}$ tube and centrifuged at $1000 \mathrm{~g}$ for 
$3 \mathrm{~min}$. The supernatant was discarded and the pellet resuspended in myotube anti-differentiation medium (PMT anti-diff medium) to prevent cells for differentiation. Cells were splitted at the rate 1:4. Freezing and thawing of primary myotubes was performed as it is described for MEFs / REFs (2.2.2.2.). Medium was changed every second day.

Myotube anti-differentiation medium (PMT anti-diff medium):

- HAM F12

- $20 \%$ FBS

- $1 \%$ Antibiotic Antimycotic

- $1 \%$ GlutaMax $^{\mathrm{TM}}$

- $2.5 \mathrm{ng} / \mathrm{ml}$ FGF2

\subsubsection{Differentiation of primary myotubes}

Medium was changed to myotube differentiation medium (PMT diff medium) when myoblasts had reached about $70 \%$ confluence. After 5-7 days myoblasts fused to myotubes and after additional 5-7 days spontaneous contractions could be observed. Medium was changed every second day.

Primary myotube differentiation medium (PMT diff medium):

- $50 \%$ DMEM $2 x$

- $28 \%$ destilled $\mathrm{H}_{2} \mathrm{O}$

- $15 \%$ horse serum (heat inactivated)

- $1 \%$ Antibiotic Antimycotic

- $1 \%$ GlutaMax $^{\mathrm{TM}}$

- $1 \%$ Sodium Pyruvate

- $1 \%$ HEPES-buffer

\subsubsection{Culture of human Myotubes}

Human myoblasts were received from the EuroBioBank (European Network of DNA, Cell and Tissue Banks for Rare Disease). All samples used in this study were obtained from healthy controls. 


\subsubsection{Culture of human Myotubes}

Initially human myoblasts were cultured in human myotube proliferation medium (hMT prolif. medium) on $0.1 \%$ gelatin coated dishes. FGF2 und EGF were added to the medium to support myoblast proliferation. Myoblast cultures were passaged when they cells became $60 \%$ confluent. Myoblasts were detached by TrypLE Express digestion $\left(500 \mu \mathrm{l} /\right.$ well, $5 \mathrm{~min}$ at $\left.37^{\circ} \mathrm{C}\right)$. After incubation cells were collected in a $15 \mathrm{ml}$ tube and $5 \mathrm{ml}$ of medium were added. Cells were centrifuged at $1000 \mathrm{rpm}$ for $7 \mathrm{~min}$. Subsequently, $1.5 \times 10^{5}$ cells were plated per well of a 6 well-plate and cultured in $5 \% \mathrm{CO}_{2}$ and $95 \% \mathrm{O}_{2}$. Freezing and thawing of human myoblasts was performed as described for MEFs.

Human myotube proliferation medium (hMT prolif. medium):

- HAM F12

- $20 \%$ FBS

- $1 \%$ Antibiotic Antimycotic

- $1 \%$ GlutaMax $^{\mathrm{TM}}$

- $1 \%$ Insulin

supplemented with:

- $10 \mathrm{ng} / \mathrm{ml}$ FGF2

- $10 \mathrm{ng} / \mathrm{ml}$ EGF

\subsubsection{Differentiation of human Myotubes}

To induce differentiation of human myoblasts into myotubes, cells were cultured until they reached about $80 \%$ confluence. At this time point medium was changed to human myotube differentiation medium (hMT diff medium). After 10 days, myoblasts started to fuse to polynuclear, elongated myotubes.

Human myotube differentiation medium (hMT diff medium):

- HAM F12

- $5 \%$ horse serum

- $1 \%$ Antibiotic Antimycotic

- $1 \%$ Insulin 


\subsubsection{Co-culture of primary / human Myotubes and Motoneurons}

2.2.9.2. Co-culture of primary / human Myotubes and hiPSCs derived Motoneurons

For co-culture experiments, myoblasts were plated onto PLO and laminin coated treated ibidis (35 $\mathrm{mm}$ high, tissue culture treated) (coating procedure is described in 2.2.4.1.). Myotubes were differentiated into polynuclear and elongated myotubes. After differentiation, pMNs at day 38 of differentiation were seeded onto the myotube layer and cultured in co-culture medium. Half of the medium was changed once a week.

Co-culture medium:

- Basic differentiation medium

- $2 \%$ Serum Replacement

supplemented with:

- $10 \mathrm{ng} / \mathrm{ml}$ GDNF

- $10 \mathrm{ng} / \mathrm{ml}$ BDNF

- 10 ng/ml IGF1

- $0.1 \mu \mathrm{M}$ cAMP

- $20 \mu \mathrm{g} / \mathrm{ml}$ Ascorbic Acid

\subsubsection{Co-culture of primary Myotubes and rat Motoneurons}

Myotube culture was performed as described above. Primary rat motoneurons were seeded onto myotubes directly after preparation and cultured in primary coculture medium. Half of the medium was changed once a week.

Primary co-culture medium:

- $33 \%$ DMEM

- $66 \%$ knockout DMEM F12

- $2 \%$ Serum Replacement

- $1 \%$ NEAA

- $1 \%$ Antibiotic Antimycotic

- $1 \%$ GlutaMax ${ }^{\mathrm{TM}}$

- $2 \%$ B27 without Vit.A 
supplemented with:

- $10 \mathrm{ng} / \mathrm{ml}$ GDNF

- $10 \mathrm{ng} / \mathrm{ml} \mathrm{BDNF}$

\subsubsection{Inhibition of neuromuscular Junctions}

Experiments were performed with motoneurons seeded on 2 week old human myotubes / primary myotubes and co-cultured for around 10 days. Contraction of myotubes was measured under the life-cell microscope shortly before adding the toxins. Pancuronium ( $3 \mu \mathrm{M}$, Pancuronium-duplex, Actavis, Hafnarfjordur, Iceland) as well as botulinumtoxin A (5 Units, BOTOX, Allergan, Irvine, CA, USA) were diluted in warm co-culture medium (without any supplements). The medium was removed and the toxins were immediately applied to the cells. Contractions of the myotubes were filmed using an inverse life-cell microscope (Axiovert $200 \mathrm{M}$, Zeiss, Oberkochen, BW, Germany). Medium was changed 30 min after toxin applications and the cells were kept in culture again.

\subsection{Virus production}

\subsubsection{Viral construct}

Keratinocytes from plucked human hair were reprogrammed with a lentiviral polycystronic STEMCCA cassette carrying OCT4, SOX2, KIF4 and C-MYC [178]. Additionally the vector is flanked by loxP sites at the 3' LTR and also at the 5' LTR end to enable CRE-mediated excision of the STEMCCA cassette. The coincident expression of all four transcription factors results in a high reprogramming efficiency.

\subsubsection{Production of the STEMCCA Lentivirus}

Lenti-X cells were splitted one day prior transfection and $4-5 \times 10^{6}$ cells were seeded on $10 \mathrm{~cm}$ dishes and cultured in $10 \mathrm{ml}$ Lenti-X medium. Cells were 50-80 $\%$ confluent at the day of transfection. For each $10 \mathrm{ml}$ dish the following mixture was prepared: 
Transfection-mixture for virus production:

- $400 \mu \mathrm{l}$ Opti-MEM (invitrogen)

- $8 \mu \mathrm{g}$ StemCCA-vector DNA

- $5.5 \mu \mathrm{g}$ psPAX2-vector DNA

- $2 \mu \mathrm{g}$ pMD2-vector DNA

- $70 \mu \mathrm{l} \mathrm{PEI}$ (Polyethylenimine)

Transfection mixture was incubated for $10 \mathrm{~min}$ at room temperature. Meanwhile the old medium of the Lenti-X cells was replaced by $6.5 \mathrm{ml}$ Lenti-X medium. After incubation, the transfection mixture was carefully added to the cells and cells were incubated for $4 \mathrm{~h}$ at $37^{\circ} \mathrm{C}$. After 4 hours medium was replaced by $8 \mathrm{ml}$ Lenti- $\mathrm{X}$ medium. Two days after the initial transfection, the medium was harvested and stored at $4^{\circ} \mathrm{C}$ or $-80^{\circ} \mathrm{C}$. Subsequently, $8 \mathrm{ml}$ fresh Lenti-X cell medium were applied to the cells. After two additional days medium was again harvested and mixed up with the medium which had been harvested after $48 \mathrm{~h}$ of transfection.

\subsubsection{Concentration of the viral Supernatant}

The harvested lentiviral supernatant was centrifuged at $1500 \mathrm{rpm}$ for 2 min. After centrifugation the supernatant was sterile filtered using a $0.45 \mu \mathrm{m}$ filter (nitrocellulose filter should not be used) and mixed with the Viral Concentrator solution (Clontech) in the ratio 3:1. Then, the mixture was stored at $4^{\circ} \mathrm{C}$ over night. At the next day, the concentrated viral supernatant was centrifuged at $1500 \mathrm{rpm}$ for $45 \mathrm{~min}$ at $4{ }^{\circ} \mathrm{C}$. After centrifugation, the supernatant was decanted and the pellet was resupended in Epilife. The viral pellet of one $10 \mathrm{ml}$ dish was resuspended in $1 \mathrm{ml}$ Epilife. Aliquots $(1 \mathrm{ml})$ were stored at $-80^{\circ} \mathrm{C}$.

\subsubsection{Quantification of the virion Content of lentiviral Supernatant}

For quantification of the virion content of the lentiviral supernatant, Lenti- $X^{\mathrm{TM}}$ qRTPCR Titration Kit (Clontech) was used. Viral supernatant was collected and concentrated as described above. Genomic viral RNA was purified and then treated with DNase I to remove any residual plasmid DNA that may have been carried over from transient transfection of the packing cells. Serial dilutions of the viral RNA samples have been analyzed by qRT-PCR (quantitative real time reverse transcription-polymerase chain reaction, described in detail in 2.5.) to 
determine crossing points (CP) for each dilution. The RNA genome copy number in a sample was determined by finding the copy number that corresponds to its $\mathrm{CP}$ value on a standard curve generated from serial dilutions of the calibrated Lenti- $X$ RNA Control Template.

\subsubsection{Viral genomic RNA Isolation}

Before starting the preparation, an aliquot of Elution Buffer RE and of RNase-free $\mathrm{H}_{2} \mathrm{O}$ was pre-heated to $70^{\circ} \mathrm{C}$. $600 \mu \mathrm{l}$ Buffer RAV1 (containing reconstituted Carrier RNA) were added to $150 \mu \mathrm{l}$ of the viral supernatant and mixed by pipetting. Then, $600 \mu \mathrm{l} \mathrm{EtOH}$ were added and mixed by vortexing. NucleoSpin RNA Virus Columns were placed into $2 \mathrm{ml}$ collection tubes, loaded with $700 \mu \mathrm{l}$ lysed sample and centrifuged at $8,000 \times \mathrm{g}$ for $1 \mathrm{~min}$. After centrifugation, membrane was washed with $500 \mu \mathrm{l}$ Buffer RAW followed by washing with $600 \mu \mathrm{l}$ Buffer RAV3. For the $3^{\text {rd }}$ washing step, NucleoSpin RNA Virus Column was placed in a new collection tube and $200 \mu \mathrm{l}$ Buffer RAV3 were added and centrifuged at 11,000 x g for 2-5 min. NucleoSpin RNA Virus Column was then placed into a sterile $1.5 \mathrm{ml}$ collection tube and $50 \mu \mathrm{l}$ preheated RNase-free $\mathrm{H}_{2} \mathrm{O}$ (preheated to $70^{\circ} \mathrm{C}$ ) were added and incubated for 1-2min. After incubation the sample was centrifuged at $11,000 \times \mathrm{g}$ for $1 \mathrm{~min}$.

\subsubsection{DNase I Treatment}

Purified viral RNA was treated with DNase I to remove any residual plasmid DNA. A thermocycler was used for DNase I reaction.

DNase I reaction:

- $12.5 \mu$ l viral RNA

- $2.5 \mu \mathrm{l}$ DNase I Buffer 10x

- $10 \mu \mathrm{l}$ DNase I (5 units / $\mu \mathrm{l})$

- $6.0 \mu \mathrm{l}$ RNase-free $\mathrm{H}_{2} \mathrm{O}$

$\rightarrow$ reagents were mixed

$\rightarrow$ incubated for $30 \mathrm{~min}$ at $37^{\circ} \mathrm{C}$

$\rightarrow$ incubated for $5 \mathrm{~min}$ at $70^{\circ} \mathrm{C}$

$\rightarrow$ stored on ice until ready to perform qRT-PCR 


\subsubsection{QRT-PCR Amplification of lentiviral genomic RNA}

Pipetting for qRT-PCR was performed on ice using a pre-cooled PCR-plate. For qRT-PCR measurements each calibrated control, no-template control (NTC) and at least 3 different purified viral RNA samples were performed as duplicates. RT (reverse transcriptase) enzyme was added immediately before qRT-PCR starts.

QRT-PCR mixture:

- $2 \mu \mathrm{R}$ RA of each control and sample dilution

- $8.5 \mu \mathrm{R}$ Rase-free $\mathrm{H}_{2} \mathrm{O}$

- $12.5 \mu \mathrm{l}$ Quant-X Buffer 2x

- $0.5 \mu$ L Lenti-X Forward Titer Primer $(10 \mu \mathrm{M})$

- $0.5 \mu$ l Lenti-X Reverse Titer Primer $(10 \mu \mathrm{M})$

- $0.5 \mu$ l Quant-X Enzyme

- $0.5 \mu \mathrm{l}$ RT Enzyme

Preparation of different control dilution was performed as followed:

\begin{tabular}{|c|c|c|c|}
\hline \multicolumn{4}{|c|}{ Control dilutions } \\
\hline Control dilution & EASY Buffer & $\begin{array}{c}\text { Lenti-X RNA } \\
\text { Control Template }\end{array}$ & $\begin{array}{c}\text { Amount } \\
\text { (copies } / \mu l)\end{array}$ \\
\hline$\underline{1}$ & $18 \mu \mathrm{l}$ & $2 \mu \mathrm{l}$ & $5 \times 10^{\prime}$ \\
\hline$\underline{2}$ & $27 \mu \mathrm{l}$ & $3 \mu \mathrm{l}$ of Dilution 1 & $5 \times 10^{6}$ \\
\hline$\underline{3}$ & $27 \mu \mathrm{l}$ & $3 \mu \mathrm{l}$ of Dilution 2 & $5 \times 10^{5}$ \\
\hline$\underline{4}$ & $27 \mu \mathrm{l}$ & $3 \mu \mathrm{l}$ of Dilution 3 & $5 \times 10^{4}$ \\
\hline$\underline{5}$ & $27 \mu \mathrm{l}$ & $3 \mu \mathrm{l}$ of Dilution 4 & $5 \times 10^{3}$ \\
\hline$\underline{6}$ & $10 \mu \mathrm{l}$ & - & $\mathrm{NCT}$ \\
\hline$\underline{7}$ & $10 \mu \mathrm{l}$ & - & $\mathrm{NCT}$ \\
\hline$\underline{8}$ & $10 \mu \mathrm{l}$ & - & $\mathrm{NCT}$ \\
\hline
\end{tabular}

Each dilution was mixed well by tapping gently. $2 \mu \mathrm{l}$ of each dilution have been used for qRT-PCR measurements. 
The qRT-PCR was set up with the following program:

\begin{tabular}{|c|c|c|c|}
\hline Step & Temperature & Duration & Cycles \\
\hline $\begin{array}{c}\text { Reverse } \\
\text { Transcription }\end{array}$ & $42^{\circ} \mathrm{C}$ & $5 \mathrm{~min}$ & 1 \\
\hline Denaturation & $95^{\circ} \mathrm{C}$ & $5 \min$ & 1 \\
\hline Denaturation & $95^{\circ} \mathrm{C}$ & $5 \mathrm{~s}$ & 40 \\
\hline Amplification & $60^{\circ} \mathrm{C}$ & $30 \mathrm{~s}$ & 40 \\
\hline $\begin{array}{l}\text { melting curve } \\
\text { analysis }\end{array}$ & $\begin{array}{l}\text { ramp from } 60^{\circ} \mathrm{C} \text { to } \\
95^{\circ} \mathrm{C}\left(1^{\circ} \mathrm{C} / \text { step }\right)\end{array}$ & $\begin{array}{c}90 \mathrm{~s} \text { (first step) } \\
5 \mathrm{~s} \text { (following } \\
\text { steps) }\end{array}$ & \\
\hline cooling & $40^{\circ} \mathrm{C}$ & $2 \min$ & 1 \\
\hline
\end{tabular}

NucleoSpin Viral RNA Isolation Kit:

- Lyses Buffer RAV1

- Wash Buffer RAW

- Wash Buffer RAV3 (Concentrate)

- RNase-free $\mathrm{H}_{2} \mathrm{O}$

- Elution Buffer RE

- Carrier RNA (lyophilized)

- NucleoSpin RNA Virus Columns

- Collection Tubes (2 ml)

Lenti-X qRT-PCR Components:

- Lenti-X RNA Control Template $\left(5 \times 10^{8}\right.$ copies / $\mu$ l)

- DNase I (5 units / $\mu$ l)

- DNase I Buffer 10x

- Lenti-X Forward Titer Primer $(10 \mu \mathrm{M})$

- Lenti-X Reverse Titer Primer $(10 \mu \mathrm{M})$

- EASY Dilution Buffer

\subsubsection{Data Analysis}

A standard curve is created from the CP values of the different control dilutions ( $y$ axis $=C P$ values, $x$-axis $=$ amount of copies $/ \mu \mathrm{l}$, log-scale). For each sample, copy number was back-calculated:

Copies $/ \mathbf{m l}=\frac{\left((\text { copies value }) *\left(100 \mu \frac{l}{\mathrm{ml}}\right) *(20 \mu l \text { DNAse I }) *(50 \mu l \text { elution })\right)}{(150 \mu l \text { sample }) *(2 \mu l \text { added per tube })}$ 


\subsection{Protein biochemistry}

\subsubsection{Immunocytochemistry}

The protocol was obtained from Liebau et al. [107]. For immunofluorescence analysis cells were either plated on glass cover slides or on treated ibidis. At first, the medium was poured off and the cells were washed with PBS ++. The cells were further fixed in $4 \%$ PFA (paraformaldehyde) / $2 \%$ sucrose for $20 \mathrm{~min}$ at room temperature. After fixation, cells were washed twice with PBS ++. Permeabilization of the cell membrane was performed either with $0.2 \%$ Triton- $X$ treatment for $5 \mathrm{~min}$ at room temperature or with ice-cold $100 \%$ methanol for min at $-20^{\circ} \mathrm{C}$. Methanol permeabilization was required for following primary antibodies: OCT4, SOX2, and NANOG. Permeabilization was omitted if staining against an extracellular antibody epitope (SSEA4, TRA-1-60, TRA-1-81) was performed. After permeabilization, the blocking solution was added to the cells. Usually $5 \%$ goat serum was used for blocking ( $1 \mathrm{~h}$, at room temperature) except another blocking solution was mentioned in the datasheet of the primary antibody. The primary antibodies were diluted in the respective blocking solution and were either incubated for $1 \mathrm{~h}$ at room temperature or for $12 \mathrm{~h}$ at $4^{\circ} \mathrm{C}$ in a light proof staining chamber. The cover slips were washed in PBS ++ and in the meanwhile the secondary antibody solution was prepared in PBS - - and added to the cover slips ( $1 \mathrm{~h}$ at room temperature). Then, the cover slips were washed in PBS ++ for $5 \mathrm{~min}$, followed by 5 min washing in PBS - - and subsequently in $\mathrm{H}_{2} \mathrm{O}$ bidest for 5 min. After washing procedure, cover slips were the dripped off on a tissue paper and then laid on an objective slide on which a droplet of Prolong antifade reagent with/without Dapi (Invitrogen) had been applied before. Dapi staining was performed when staining cells cultured in ibidis. Dapi was diluted 1:20,000 in PBS - - and added to the cells after PBS ++ washing step for $3 \mathrm{~min}$ at room temperature. Afterwards, cells were washed in PBS - - and $\mathrm{H}_{2} \mathrm{O}$ bidest. Carefully 1-2 droplets of Prolong antifade reagent without Dapi (Invitrogen) were added to the Ibidi surface. Analysis was done with a fluorescent microscope (Axiokop 2, Zeiss) equipped with a Zeiss CCD camera (16 bits; 1280 x 1024 pixels per image), and using Axiovision software (Zeiss). 
Fixation solution $4 \%$ PFA / $2 \%$ Sucrose:

- $8 \mathrm{~g}$ paraformaldehyde (PFA)

$\rightarrow$ PFA was dissolved in $80 \mathrm{ml}$ deionized $\mathrm{H}_{2} \mathrm{O}$ at $57^{\circ} \mathrm{CC}$

- $4 \mathrm{~g}$ sucrose

$\rightarrow$ sucrose was dissolved in $100 \mathrm{ml}$ PBS - -

$\rightarrow$ both solution were pooled

$\rightarrow \mathrm{pH} 7.4$

$0.2 \%$ Triton- $X$ :

- $100 \mu \mathrm{l}$ Triton-X 100x (Roche)

- $50 \mathrm{ml}$ PBS + +

$\rightarrow$ Triton-X was dissolved at $4^{\circ} \mathrm{C}$ over night

Blocking Solution:

- $20 \%$ Goat Serum

- $\mathrm{PBS}++$

\subsubsection{Flow Cytometry Analysis (FACS) for intracellular Markers}

Cells were detached by $0.125 \%$ Trypsin-Versen digestion for $10 \mathrm{~min}$ at $37^{\circ} \mathrm{C}$. Cells were then collected in a $15 \mathrm{ml}$ tube, centrifuged at $1500 \mathrm{rpm}$ for $2 \mathrm{~min}$ and washed in FACS buffer. Cells were counted using a Neubauer counting chamber and at least 100,000 cells per antibody had been used. Afterwards, the cells were fixed with $4 \%$ PFA / $2 \%$ sucrose for 20 min on ice. Next, cells were permeabilized in $0.5 \%$ saponin for $20 \mathrm{~min}$ on ice. In the meanwhile, the primary antibody (SMI32; $1: 500)$ was diluted in PBS $+0.1 \%$ saponin. After permeabilization, cells were pelleted and resuspended in the primary antibody solution. The primary antibody cell suspension was then pipetted into the wells of a 96 well u-bottom plate and incubated for $1 \mathrm{~h}$ at $4^{\circ} \mathrm{C}$ under constant agitation. Subsequently, cells were washed three times and resuspended in the secondary antibody (diluted in PBS - , 1:500) for $1 \mathrm{~h}$ at $4^{\circ} \mathrm{C}$ while shaking. Corresponding to each secondary antibody a control had to be performed stained with the secondary antibody but not with the primary antibody. Cells were washed twice, resuspended in buffer and filtered through a $70 \mu \mathrm{m}$ filter into FACS tubes. Measurements were performed in a LSR II flow cytometer using FACSDiva software (both BD Bioscience). 
FACS buffer:

- $2 \%$ FBS

- PBS - -

Permeabilization solution:

- $0.5 \%$ saponin (Sigma-Aldrich)

- PBS - -

Washing solution:

- $0.1 \%$ saponin

- PBS - -

\subsection{Quantitative one-step real time reverse transcription-polymerase chain reaction}

\subsubsection{Purification of total RNA}

First of all, cells were dissociated by $0.125 \%$ Trypsin-Versen digestion. After removing the old medium and washing with PBS - - , $500 \mu \mathrm{l} 0.125 \%$ TrypsinVersen were added to the well and incubated for $10 \mathrm{~min}$ at $37^{\circ} \mathrm{C}$. Cells were then dissociated into a single-cell suspension, collected and centrifuged at $1500 \mathrm{rpm}$ for 3 min. After centrifugation cells were washed twice in PBS - -. Total RNA was obtained from at least of one confluent well of a 24-well plate.

Total RNA purification was assessed using RNeasy Mini Kit (Qiagen). Pelleted cells were resupended in $600 \mu \mathrm{l}$ RLT Buffer and homogenized by pipetting. The lysate was directly pipetted into a QIAshredder spin column (Qiagen), placed into a $2 \mathrm{ml}$ collection tube and centrifuged at full speed for $2 \mathrm{~min}$. After centrifugation the QIAshredder spin column was removed and $600 \mu \mathrm{l} 70 \% \mathrm{EtOH}$ were added to the flow-through and mixed well by pipetting. Next, $700 \mu \mathrm{l}$ of the lysate were pipetted to an RNeasy spin column placed in a $2 \mathrm{ml}$ collection tube and centrifuged at $\geq 10,000 \mathrm{rpm}$ for $15 \mathrm{~s}$. The flow-through was discarded and $700 \mu \mathrm{l}$ RW1 Buffer were added to the RNeasy spin column and centrifuged at $\geq 10,000$ rpm for $15 \mathrm{~s}$ to wash the spin column membrane. Again the flow-through was discarded, $500 \mu \mathrm{l}$ RPE Buffer were added and centrifuged at $\geq 10,000 \mathrm{rpm}$ for 15 s. Another $500 \mu \mathrm{l} \mathrm{RPE} \mathrm{Buffer} \mathrm{were} \mathrm{added} \mathrm{to} \mathrm{the} \mathrm{column} \mathrm{and} \mathrm{centrifuged} \mathrm{at} \geq$ -66 - 
$10,000 \mathrm{rpm}$ for 2 min to wash the membrane. The RNeasy spin column was then placed into a new $2 \mathrm{ml}$ collection tube and centrifuged at full speed for $1 \mathrm{~min}$. Then, the RNeasy spin column was placed in a $1.5 \mathrm{ml}$ collection tube and $30 \mu \mathrm{l}$ RNase-free water were added to the membrane and incubated for $1 \mathrm{~min}$ at room temperature. After incubation, column was centrifuged at $\geq 10,000 \mathrm{rpm}$ for $1 \mathrm{~min}$. This step was repeated so that obtained RNA was dissolved in $60 \mu \mathrm{l}$ RNase-free water. RNA samples were stored at $-80^{\circ} \mathrm{C}$.

RNeasy Mini Kit:

- RNeasy Mini Spin Columns (pink)

- QIAshredder Spin Columns (purple)

- Collection Tubes (1.5 ml)

- Collection Tubes $(2.0 \mathrm{ml})$

- Buffer RLT

- Buffer RW1

- Buffer RPE (before using the first time 4 volumes of $100 \% \mathrm{EtOH}$ had been added)

- RNase-Free Water

\subsubsection{Quantitative one-step real time reverse transcription-polymerase chain reaction}

2.5.2.1. QRT-PCR Amplification of total RNA

Quantitative one-step real time reverse transcription-polymerase chain reaction (qRT-PCR) was carried out according to $[95,107]$ using RotorGene Q System (Qiagen). Amplification was monitored and analyzed by measuring the binding of the fluorescence dye SYBR Green I to minor groove of double-stranded DNA. Primers were commercially available and listed in table 4. "One-step qRT-PCR" means that reverse transcription and amplification proceed in the same reaction resulting in a higher standardization and in reduction of the influence of external error sources. 
Table 4: QuaniTect Primers

\begin{tabular}{|c|c|c|}
\hline $\begin{array}{c}\text { QuantiTect Primer } \\
\text { Assay }\end{array}$ & Gene & Function \\
\hline NES & nestin & neural stem cell marker \\
\hline TUBB 3 & tubulin $\beta$ III & neuronal marker \\
\hline NEFH & SMl-32 & motoneuron marker \\
\hline CHAT & acetylcholinetransferase & motoneuron marker \\
\hline SYP & synaptophysin & synaptic vesicles \\
\hline BSN & bassoon & presynaptic marker \\
\hline PCLO & piccolo & presynaptic marker \\
\hline NRXN1 & neurexin 1 & $\begin{array}{c}\text { presynaptic adhesion } \\
\text { protein }\end{array}$ \\
\hline HOMER1 & & postsynaptic marker \\
\hline ProSAP1/Shank2 & & postsynaptic marker \\
\hline ProSAP3/Shank1 & PSD 95 & postsynaptic marker \\
\hline DGL4 & neuroligin 1 & $\begin{array}{c}\text { postsynaptic adhesion } \\
\text { protein }\end{array}$ \\
\hline NGLN1 & hydroxymethylbilane & housekeeping gene \\
\hline HMBS & synthase & \\
\hline
\end{tabular}

Total RNA was reversely transcribed and subsequently amplified using QuantiFast SYBR Green RT-PCR Master Mix (Qiagen). About $0.5 \mu \mathrm{mol}$ of both sense and antisense primers was added. Each RNA preparation was tested for genomic DNA contamination in a RotorGene Q RT-PCR by replacing reverse transcriptase (RT) with water. Tenfold dilutions of total RNA were used as an external standard curve. PCR efficiency results from the slope of these standard curves. Internal standards (housekeeping gene) and samples were simultaneously amplified. Pipetting procedure was performed on ice using a pre-cooled PCR plate. RT enzyme was added immediately before starting the RotorGene program.

RotorGene Q qRT-PCR mixture:

- $1 \mu$ total RNA

- $2 \mu \mathrm{l}(0.5 \mu \mathrm{mol})$ QuantiTect Primer

- $10 \mu \mathrm{l}$ QuantiFast SYBR Green RT-PCR Master Mix

- $0.2 \mu \mathrm{RT}$ Enzyme 
The qRT-PCR was set up with the following program:

\begin{tabular}{|c|c|c|c|}
\hline Step & Temperature & Duration & Cycles \\
\hline $\begin{array}{c}\text { Reverse } \\
\text { Transcription }\end{array}$ & $50^{\circ} \mathrm{C}$ & $10 \min$ & 1 \\
\hline Denaturation & $95^{\circ} \mathrm{C}$ & $5 \min$ & 1 \\
\hline Denaturation & $95^{\circ} \mathrm{C}$ & $10 \mathrm{~s}$ & 40 \\
\hline Amplification & $60^{\circ} \mathrm{C}$ & $30 \mathrm{~s}$ & 40 \\
\hline $\begin{array}{l}\text { melting curve } \\
\text { analysis }\end{array}$ & $\begin{array}{l}\text { ramp from } 60^{\circ} \mathrm{C} \text { to } \\
95^{\circ} \mathrm{C}\left(1^{\circ} \mathrm{C} / \text { step }\right)\end{array}$ & $\begin{array}{c}90 \mathrm{~s} \text { (first step) } \\
5 \mathrm{~s} \text { (following } \\
\text { steps) }\end{array}$ & \\
\hline cooling & $40^{\circ} \mathrm{C}$ & $2 \min$ & 1 \\
\hline
\end{tabular}

\subsubsection{Relative Quantification}

Relative quantification is based on internal reference genes to determine folddifferences in expression of the target gene. Transcript levels of the different target genes were shown relatively to mRNA level of the internal housekeeping gene hydroxymethylbilane synthase (HMBS).

$\begin{array}{ll}\boldsymbol{C}_{\text {Target }} & =10^{(\text {CP-Intercept }) /(\text { Slope })} \\ \boldsymbol{C}_{\text {HMBS }} & =10^{(\text {CP-Intercept }) /(\text { Slope })} \\ \boldsymbol{C}_{\text {relative }} & =C_{\text {Target }} / C_{H M B S}\end{array}$

$C_{\text {Target }}=$ concentration of the target gene

$C_{\text {HMBS }}=$ concentration of HMBS

$C_{\text {relative }}=$ relative concentration

slope $\quad=$ slope of the standard curve; defined at -3.33

Intercept $=$ calculated $C P$ respective log-concentration of RNA-amount diluted 1:1; defined at 15

The general principle of cDNA quantification by qRT-PCR is based on plotting fluorescence against the number of cycles on a logarithmic scale. The threshold for detection of cDNA-caused fluorescence was set using RotorGene Software. The number of cycles (x-axis value) at the increase of fluorescence signal at the beginning of the exponential amplification phase (Log-Phase) is called Crossing Point (CP). The CP depends on the starting amount of the RNA template. 


\subsubsection{PCR-Efficiency}

Amplification efficiency is often variable among primers and templates. Therefore a standard curve was identified by titration of serial dilutions of the RNA template. The slope of the standard curve is used to calculate concentration of total RNA levels of the target gene. Furthermore the slope is used to determine the efficiency of amplification $(\Sigma)$.

$\Sigma: 10^{-1 / \text { slope of the standard curve }}$

$\Sigma=2.00 \quad 100 \%$ efficiency; duplication of the PCR-product per each cycle

$\Sigma>2.00$ amplification of by-products

$\Sigma<2.00$ reduced amplification of the PCR-product

\subsubsection{Melting curve analysis}

The specificity of the primers in binding to the target RNA sequence was evaluated by analysis of the primer specific melting curve. Each amplified product can assigned to a specific melting curve caused by product length and CG-amount.

\subsection{Transmission Electron Microscopy (TEM)}

Cells were fixed in $0.1 \mathrm{M}$ phosphate buffer $\mathrm{pH} 7.3$, containing $2.5 \%$ glutaraldehyde, $1 \%$ sucrose and osmicated for $1 \mathrm{~h}$ in $2 \%$ OsO4 (osmium tetroxid). Afterwards they were dehydrated in graded series of ethanol, contrasted in $2 \%$ uranyl acetate and embedded in epoxy resin (Sigma) at $60^{\circ} \mathrm{C}$. Thin sections of 70-80 nm were cut with a diamond knife on a Reichert ultramicrotome and collected on 300 mesh grids. The sections were contrasted with $0.3 \%$ lead citrate for $1 \mathrm{~min}$ and analyzed on the transmission electron microscope EM 10 (Zeiss) at $80 \mathrm{kV}$.

TEM fixation solution:

- $0.1 \mathrm{M}$ phosphate buffer

- $2.5 \%$ glutaralaldehydt

- $1 \%$ sucrose

- $\mathrm{pH} 7.3$ 


\subsection{Electrophysiology}

Electrophysiological experiments were performed as previously described [105, 118]. Briefly, hiPSC derived motoneurons were used for experimentation after differentiation for 3-6 weeks. Changes of membrane current or voltage were recorded in the whole-cell recording mode using an EPC-9 amplifier and TIDA software (HEKA, Lambrecht, Germany). Before recording, the cells were rinsed twice with an extracellular standard solution. Patch pipettes were drawn from borosilicate glass with tip resistances between 3 and $6 \mathrm{M} \Omega$ when filled with the internal standard solution. For the estimation of spontaneous synaptic currents the internal solution was composed according to a previous report in order to facilitate discrimination between excitatory (inward current) and inhibitory events (outward current) [105]. Synaptic currents were reversibly inhibited by $25 \mu \mathrm{M} \mathrm{CNQX} \mathrm{(6-}$ cyano-7-nitroquinoxaline-2,3-dione), an AMPA receptor antagonist and AP-5 (amino-5-phosphonovaleric acid), an NMDA receptor inhibitor. To improve sealing, tips were briefly dipped into $2 \%$ dimethylsilane dissolved in dichlormethane. All experiments were performed at room temperature.

Extracellular standard solution:

- $140 \mathrm{mM} \mathrm{NaCl}$

- $2.7 \mathrm{mM} \mathrm{KCl}$

- $1.5 \mathrm{mM} \mathrm{CaCl}_{2}$

- $10 \mathrm{mM}$ glucose

- 12 mM HEPES

- $\mathrm{pH} 7.3$

Internal standard solution:

- $140 \mathrm{mM} \mathrm{KCl}$

- $2 \mathrm{mM} \mathrm{MgCl} 2$

- 2 mM ATP x 2 mM Na

- $1 \mathrm{mM} \mathrm{CaCl}_{2}$

- 10 mM EGTA (thylene glycol tetraacetic acid)

- 10 mM HEPES

- $\mathrm{pH} 7.2$ 
Internal solution for estimation of synaptic currents:

- 140 mM Kgluconat

- $2 \mathrm{mM} \mathrm{MgCl}_{2}$

- 2 mM ATP x 2 mM Na

- $1 \mathrm{mM} \mathrm{CaCl}_{2}$

- $10 \mathrm{mM}$ EGTA (ethylene glycol tetraacetic acid)

- 10 mM HEPES

- $\mathrm{pH} 7.2$

\subsection{Data Analysis and Statistics}

\subsubsection{Counting of Synapses}

For counting analysis AxioVision Rel. 4.8 Software was used. Juxtaposed signals of presynaptic markers (synaptophysin and piccolo) and postsynaptic markers (HOMER1 and ProSAP1) were considered to be synapses. Synapse number was counted per $30 \mu \mathrm{m}$ dendrite length.

\subsubsection{Measurement of dendritic / axonal Lengths}

For measurement of dendritic and axonal length AxioVision Rel. 4.8 Software was used. To distinguish between the dendrites and the axon, double-stainings against MAP2 (microtubule-associated protein 2) and SMI-32 have been performed. Whereas SMI-32 is located both in the dendritic and in the axonal compartment, MAP2 is only expressed in the dendrites. Consequently, the neurite only stained with SMI-32 was considered to be the motoneuron axon.

\subsubsection{Statistical Tests}

Statistical analyses were performed using $\mathrm{X} 2$ (Fishers exact test), Student's t-test or using one-way analysis of variance (ANOVA test) with post-hoc Bonferroni's multiple comparison tests as appropriate using SPSS 18.0 software package (SPSS Inc., Chicago, IL). Results are provided as mean values \pm standard error of the mean (s.e.m.).Statistical significance was represented as follows:

$\mathrm{p}<0.5={ }^{*} ; \mathrm{p}<0.01=^{* *} ; \mathrm{p}<0.001={ }^{* *}$ 
Results

\section{Results}

\subsection{Generation of hiPSCs of human Keratinocytes}

Keratinocytes from plucked human hair were successfully and efficiently harvested, transduced and reprogrammed with a lentiviral polycistronic STEMCCA cassette, carrying the four Yamanaka-factors [192] OCT4, SOX2, KIF4 and c-MYC (Fig. 4A). The exact reprogramming procedure is described in 2.2.1.4. Reprogramming of human keratinocytes. Virus-free hiPSC lines were generated by incubating hiPSCs with recombinant CRE-protein. All hiPSCs lines were cultured under feeder-free conditions in mTeSR1 medium. In this study, 4 different hiPSCs lines have been used. Generated human iPSCs were further characterized. Beside morphology, hiPSCs expressed all major markers of pluripotency (Fig. 4B) and were able to differentiate into all three germ layers represented here by TUJ1 (ectoderm), a-Actinin (mesoderm) and AFP (endoderm) (Fig. 4C). 


\section{Results}

A

plucked

human hair

keratinocytes

infected keratinocytes

seeded on feeder cells

hiPSC
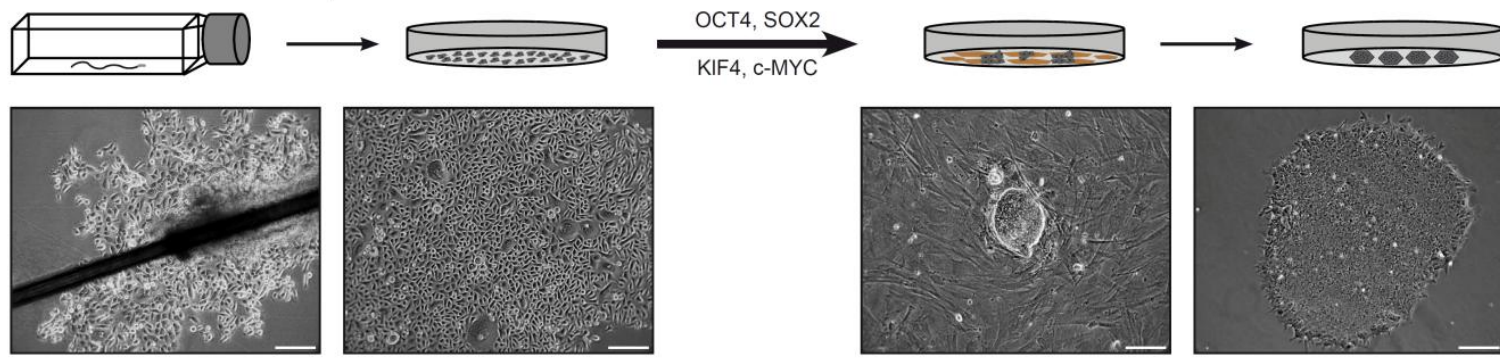

KIF4, c-MYC
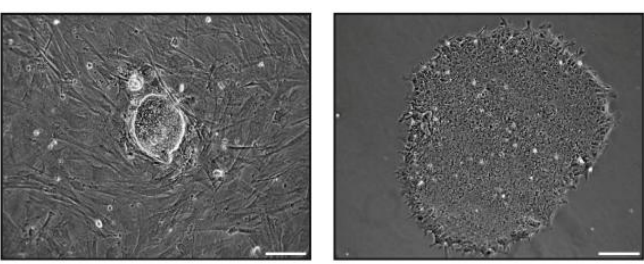

B
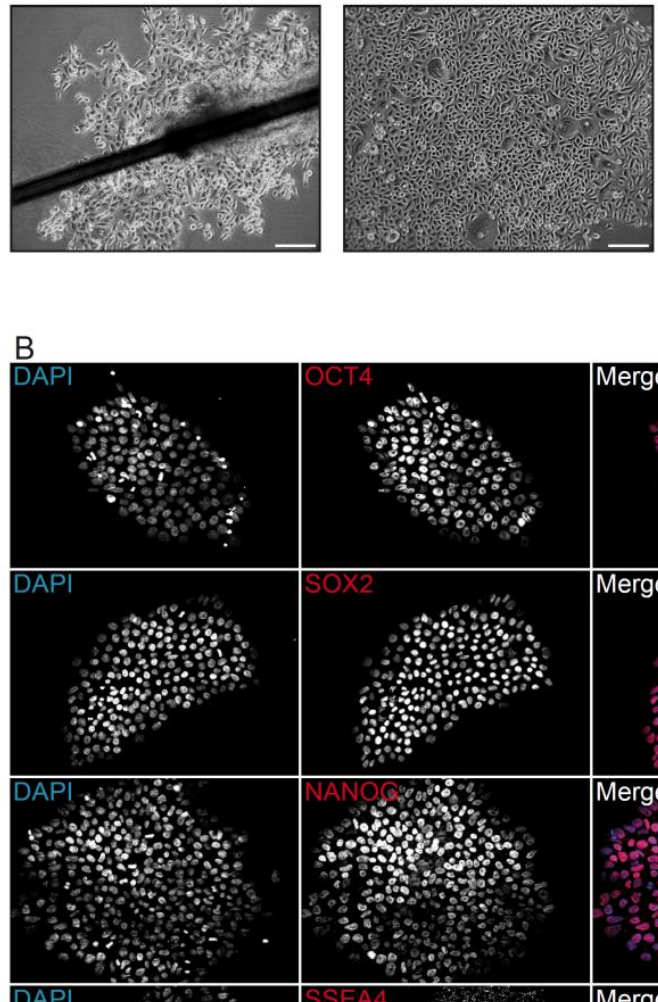

C
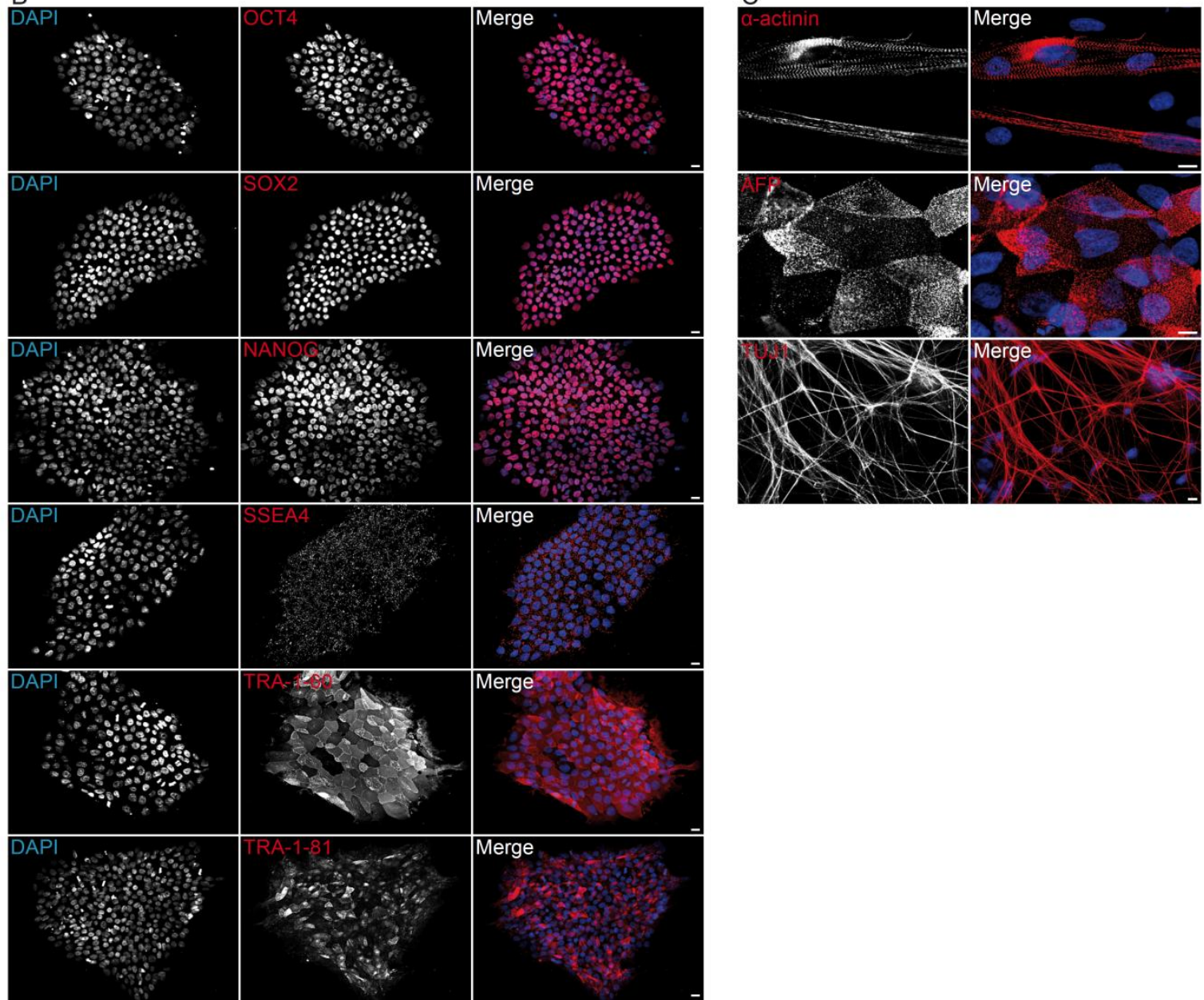

Figure 4 Generation of human induced pluripotent Stem Cells from Keratinocytes

(A) Scheme of keratinocytes reprogramming from human plucked hair into induced pluripotent stem cells (hiPSC). Brightfield microscopy images of keratinocytes emerging from the outer rout sheath of a plucked human hair. Keratinocytes were subsequently infected with a lentivirus transducing a multicistronic cassette harboring OCT4, SOX2, KIF4 and c-MYC on two subsequent days. The next day infected cells were split onto irradiated feeder cells. Around a week later small hiPSC colonies could be detected. Finally, hiPSCs were manually picked and cultured under feeder-free conditions. All scale bars are $200 \mu \mathrm{m}$. (B) HiPSCs derived from human keratinocytes express the nuclear factors OCT4, SOX2 and NANOG as well as pluripotent surface markers SSEA4, TRA-1-60 and TRA-1-81 (all red). (C) Stem cell colonies from human keratinocytes were able to differentiate in all three primary germ layers in vitro. After formation of embryoid bodies hiPSCs spontaneously differentiate into ectoderm (TUJ1, red), mesoderm ( $\alpha$-actinin, red) and endoderm (AFP, $\alpha$-fetoprotein, red) Nuclei were labeled with DAPI (blue). All scale bars are $10 \mu \mathrm{m}$. 


\section{Results}

\subsection{Differentiation into mature Motoneurons}

Differentiation into mature and functional motoneurons was achieved using a modified protocol adapted from [82] (Fig. 5A). At the beginning we also tested an unspecific neural differentiation protocol [228] resulting in the generation of various neural subtypes including gabaergic, glutamatergic neurons and also (with a low efficiency) motoneurons. To establish a high purity culture of hiPSCs derived motoneurons we established a differentiation protocol which contains the addition of RA and $\mathrm{SHH}$. Correspondingly, transcript levels of typical motoneuron markers NEFH and CHAT were increased when applying both molecules during motoneuron differentiation (Fig. 5B). In contrast to that, the mRNA level of the unspecific neuronal marker TUBB 3 was more or less equal comparing the two conditions. RA treatment started three days after re-attaching EBs (day 10) as it has been reported that the treatment of primitive neuroepithelial cells, expressing PAX6 but not SOX1, seems to be more efficient than treatment of definitive neuroepithelial cells (SOX1 / PAX6 positive) [229]. With this regimen, a large proportion of motoneurons could be detected in the cultures ( $40 \%$ ) (Fig. 5C).

The protocol of motoneuron differentiation is described in detail in section 2.2.5.1. . The motoneuron precursor morphology changed dramatically when plated on coated dishes, promoting neuronal differentiation (Fig. 5D). In the first days after plating, motoneuron neurites started to grow out of the seeded pMNs spheres. Around 7-10 days after plating (day 28-48), the maturing motoneurons formed a dense neuronal network, which was continually increasing during culture time. Human motoneurons were kept in culture for up to 20 weeks. 


\section{Results}

A

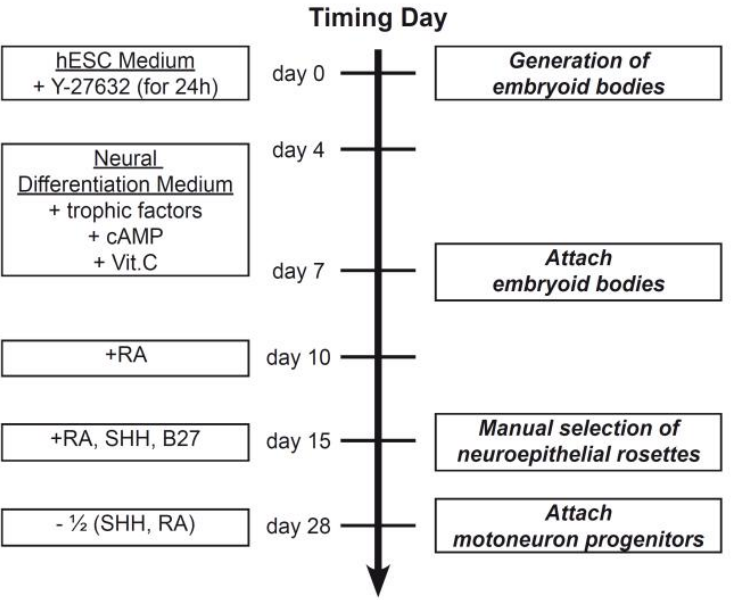

B

C

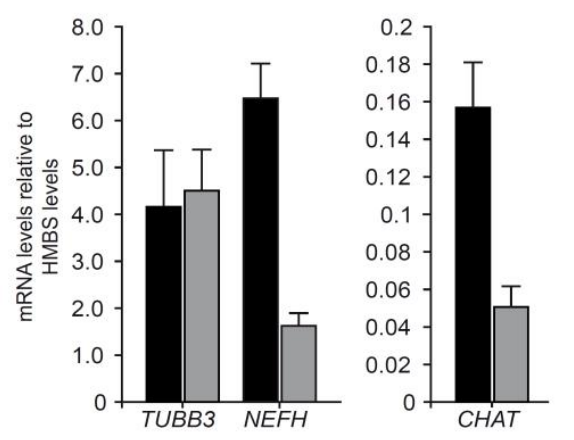

neural differentiation medium (+RA,SHH,B27 for 10 days) neural differentiation medium (+FGF2)
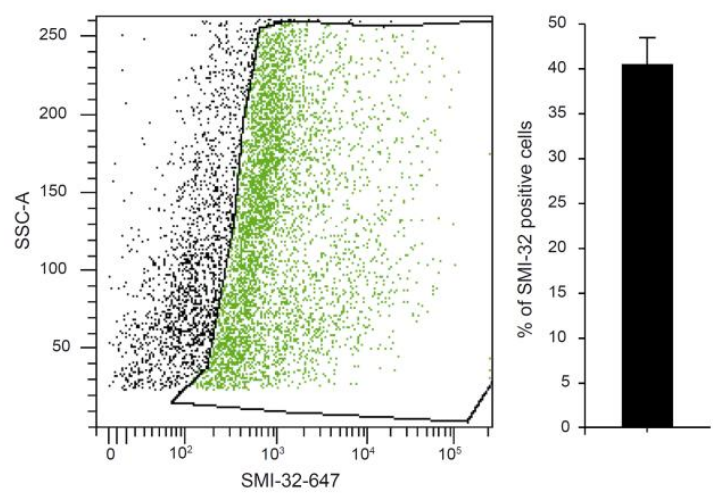

D

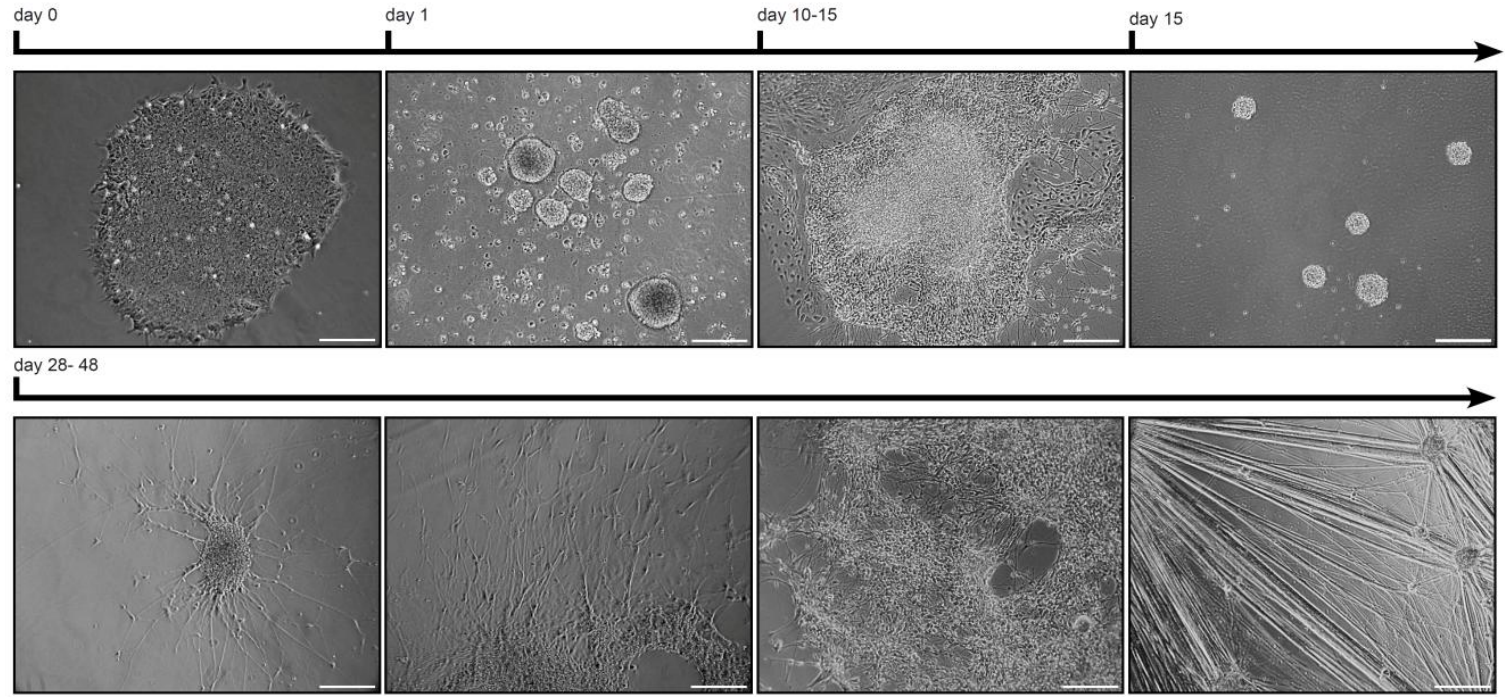

Figure 5 Differentiation of Keratinocytes derived hIPSCs into Motoneurons

(A) Schematic procedures for motoneuron (MN) differentiation. (B) SHH and RA promote proliferation and differentiation of motoneurons progenitors (pMNs, d28, $n=4$ cells per time point). (C) FACS analysis showed $40 \%$ purity of SMI-32 positive MNs ( $n=5$ cells per time point). Error bars s.e.m. (D) Bright-field imagines showing morphological changes during differentiation from hiPSCs into MNs according to differentiation scheme. 


\subsubsection{Characterization of hiPSCs derived Motoneurons}

Differentiating cell cultures were stained for several motoneuron markers i.e. SMI32, Islet1 (ISL1) and HB9 at different time points (Fig. 6A). Additionally, motoneurons expressed the vesicular acetylcholine transporter (VAChT), which mediates ACh storage by synaptic vesicles [38] and is a specific marker for "cholinergic" synaptic vesicles [209] (Fig. 6A). Furthermore, oligodendrocytes started to form a myelin sheath around the motoneurons after around 5 weeks in culture (Fig. 6C). Myelin basic protein 1 (MBP1) was used as a marker specific for myelin. Oligodendrocytes developed spontaneously in the motoneuron cultures and it is already known that gliogenesis is initiated later than neuronal development [88].

\subsubsection{Human iPSCs derived Motoneurons functionally express voltage-gated Ion Channels}

HiPSCs derived motoneurons expressed the passive membrane properties of neurons with similar characteristics after 21 and 35 days of differentiation and a resting membrane potential of $-81 \pm 7 \mathrm{mV}$ after 21 days and $-73 \pm 7 \mathrm{mV}$ after 35 days of differentiation ( $p=0.06, n=6-10)$. Upon membrane depolarization, all motoneurons differentiated for 4-5 weeks featured fast inactivating inward currents and slowly decaying outward currents, consistent with voltage-activated sodium (outward) and potassium (inward) currents (Fig. 6C). Such ion channels are essential for the generation of action potentials (AP). Consequently, APs can be induced by current injection into resting motoneurons (Fig. 6D) or detected spontaneously (Fig. 6E,F). Half power width of spontaneous generated APs was $1.65 \mathrm{msec}(n=6-10)$ and the spontaneous APs' peak was at $48.2 \mathrm{mV}(n=6-10)$. Generation of spontaneous APs was observed in $80 \%$ of all motoneurons after 35 days of differentiation, but less frequently in younger motoneurons (21 days) (Fig. $6 \mathbf{G})$. After 35 days of differentiation hiPSCs derived motoneurons expressed major electrophysiological properties of mature neurons including the repertoire of voltage-gated ion channels which is essential for the generation of APs. 


\section{Results}

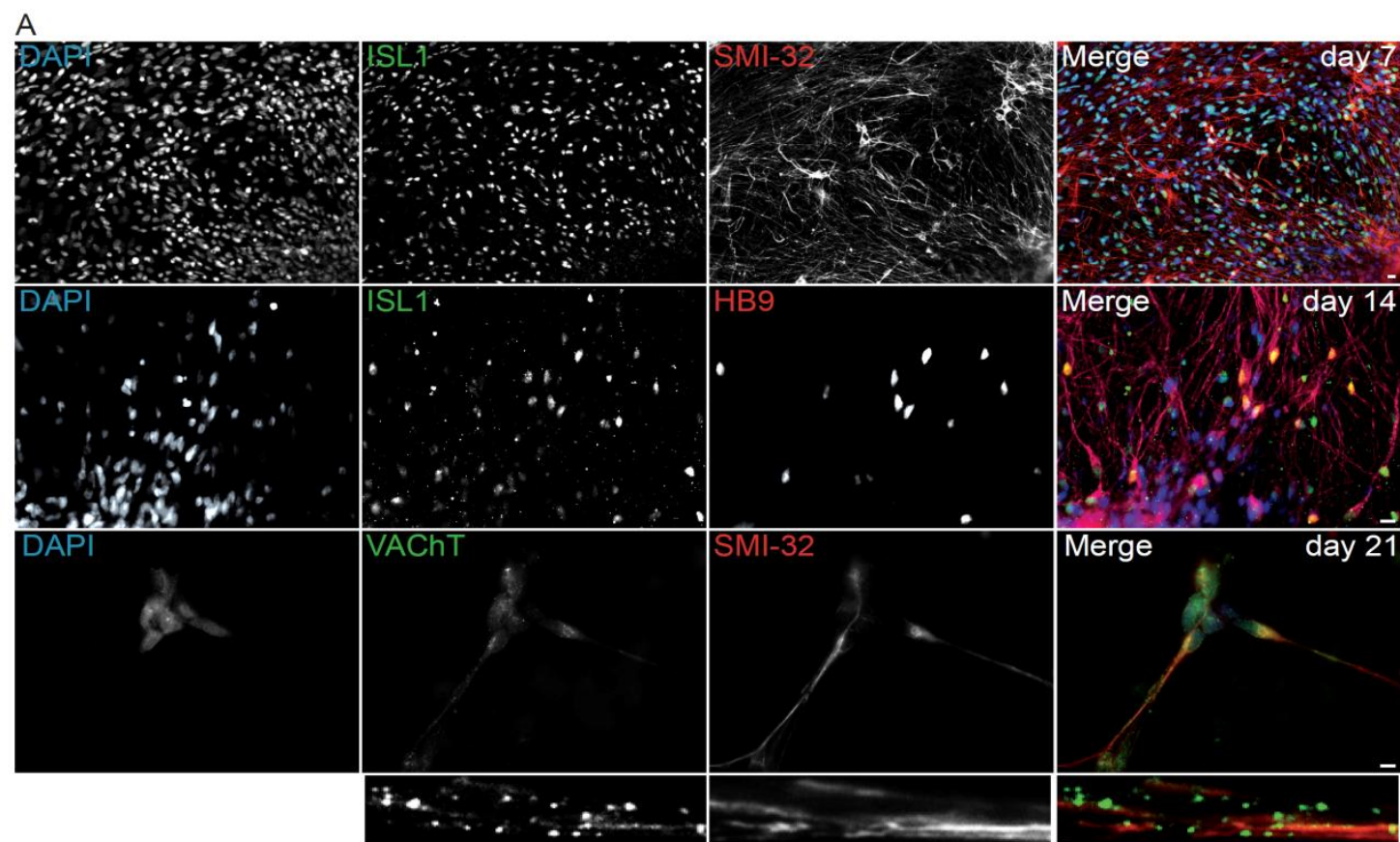

B
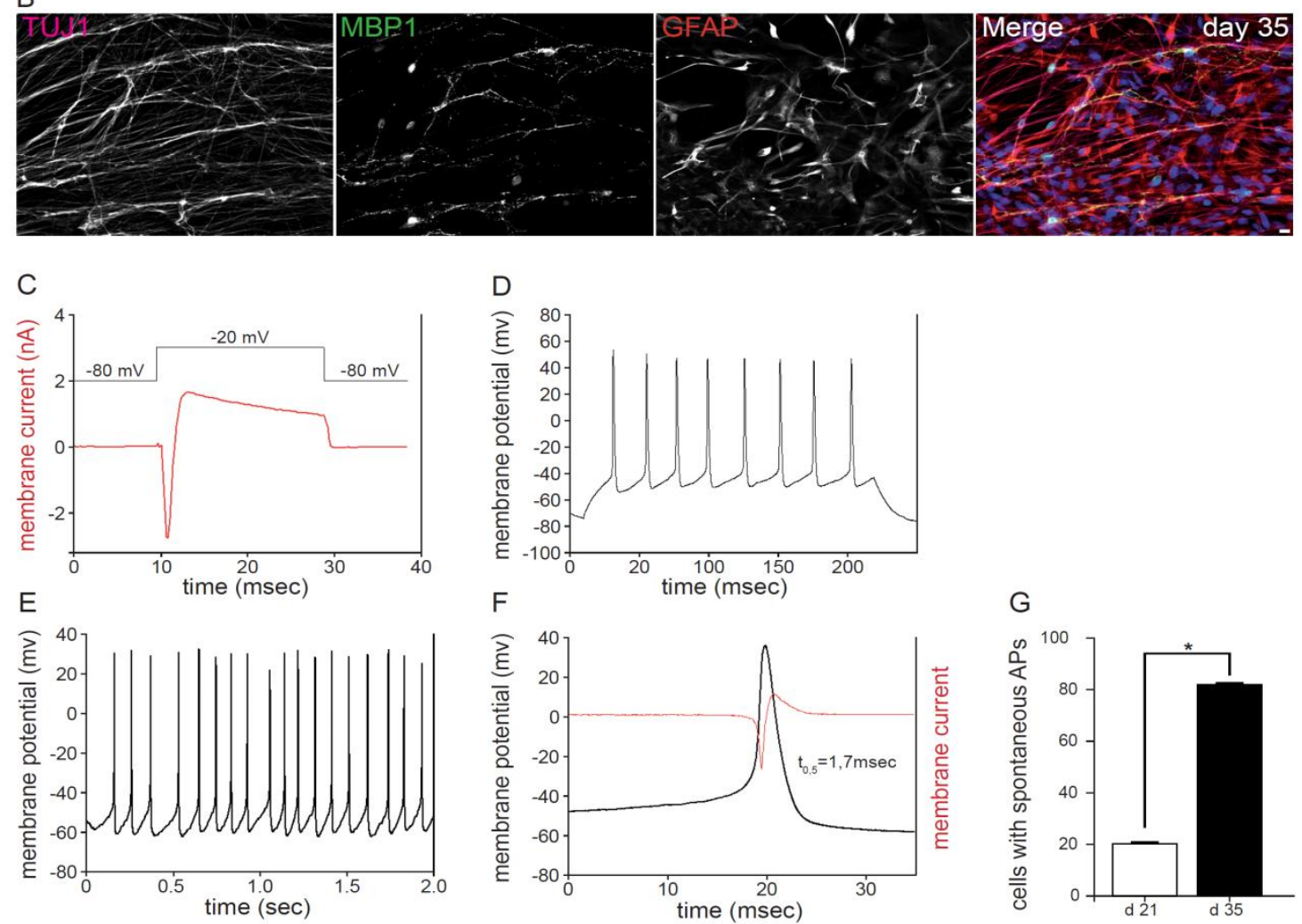

Figure 6 hIPSCs derived Motoneurons express all Signs of functional Neurons

(D) HiPSCs derived MNs expressed the subtype specific markers such as ISL1 (green), HB9 (red), VAChT (vesicular acetylcholine transporter, green) and SMI-32 (red). Additionally, MNs were surrounded by a myelin sheath of oligodendrocytes (MBP1, myelin basic protein 1, green). At later time points of differentiation a dense network of glial cells developed (GFAP, glial fibrillary acidic protein, red). Nuclei were stained with DAPI. All scale bars are $10 \mu \mathrm{m}$. (C-G) Electrophysiological characterization of hiPSCs derived MNs. After differentiation for 21 and 35 days, MNs were analyzed in the whole cell configuration of the patch-clamp recording technique using the voltage clamp (C) or current clamp (D, E, F) mode. (C) Membrane depolarization from -80 to $-20 \mathrm{mV}$ evoked fast inactivating inward-currents $\left(\mathrm{Na}^{+}\right)$followed by slowly decaying outward-currents $\left(K^{+}\right)(n=5)$. (D) Induction of action potentials by current injection (50 pA). (E) Spontaneously occurring action potentials in differentiated MNs. (F) Single spontaneous action potential: simultaneous recording of changes in voltage and current. AP duration at $0 \mathrm{mV}$ was $1.7 \mathrm{msec}$. (G) Increase of spontaneous active MNs from day 21 to day 35 ( $p=0.017$, Fishers exact test; $n=5$ cells per time point). Error bars s.e.m. 


\subsection{Distinct Maturation Steps of hiPSC derived Motoneurons}

Maturation steps of motoneuron development were primarily described according to morphological changes (Fig. 7A). Within the first 2 days after plating motoneurons started to grow out of the neurospheres and already expressed the early neuronal marker tubulin $\beta$ III (TUJ1). They exhibited a bipolar appearance with still undefined projections (day 3). This morphology was followed by a retraction of the projections and the formation of one long dominant neurite. However, this neurite was not positive for axonal markers but continually increased in length (day 7). The distal end of the neurite exhibited a growth cone like structure, building a growth and pathfinding forefront (Fig. 7B). Around the same time, 2-3 small dendrites developed. During the following weeks, further neurites were formed, branched and elongated (Fig. 7A,B).

Additionally, small filopodia-like structures could be observed along the early presumed axon (day 14) (Fig. 7B). On mRNA levels, Nestin (NES) transcript levels, marking neural progenitor cells, were continually down-regulated following an initial peak. The initial high NES mRNA level was most probably caused by an ongoing proliferation of yet undifferentiated cells. In contrast to NES mRNA levels, transcript levels for neural markers like TUBB3 (tubulin $\beta$ III) steadily increased and peaked 4 weeks after plating. The motoneuron specific marker NEFH (SMI32) showed a similar time frame of regulation as TUBB3, but peaked two weeks later and then steadily decreased. Similar to $N E F H$, marker transcripts for mature motoneurons such as choline acetylcholine transferase (CHAT, key enzyme of acetylcholine biosynthesis) sharply increased at day 28 and presented the highest mRNA level also on day 42. However, CHAT mRNA levels remained relatively high compared to TUBB3 and NEFH upon day 70 (Fig. 7C) resembling held up transmitter generation. 


\section{Results}

A

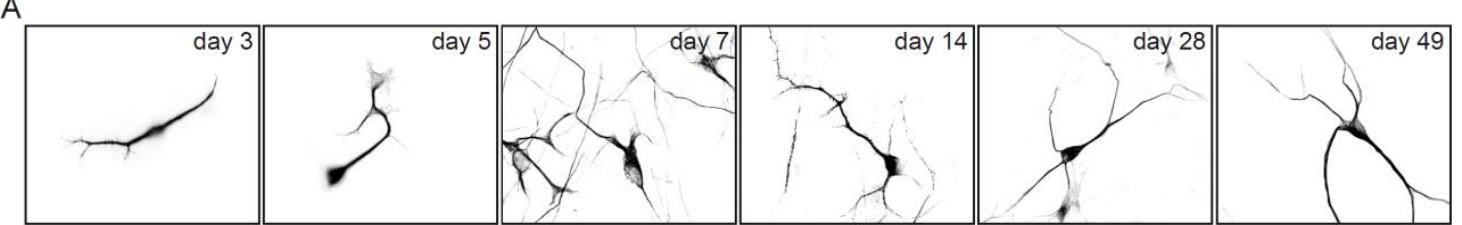

B
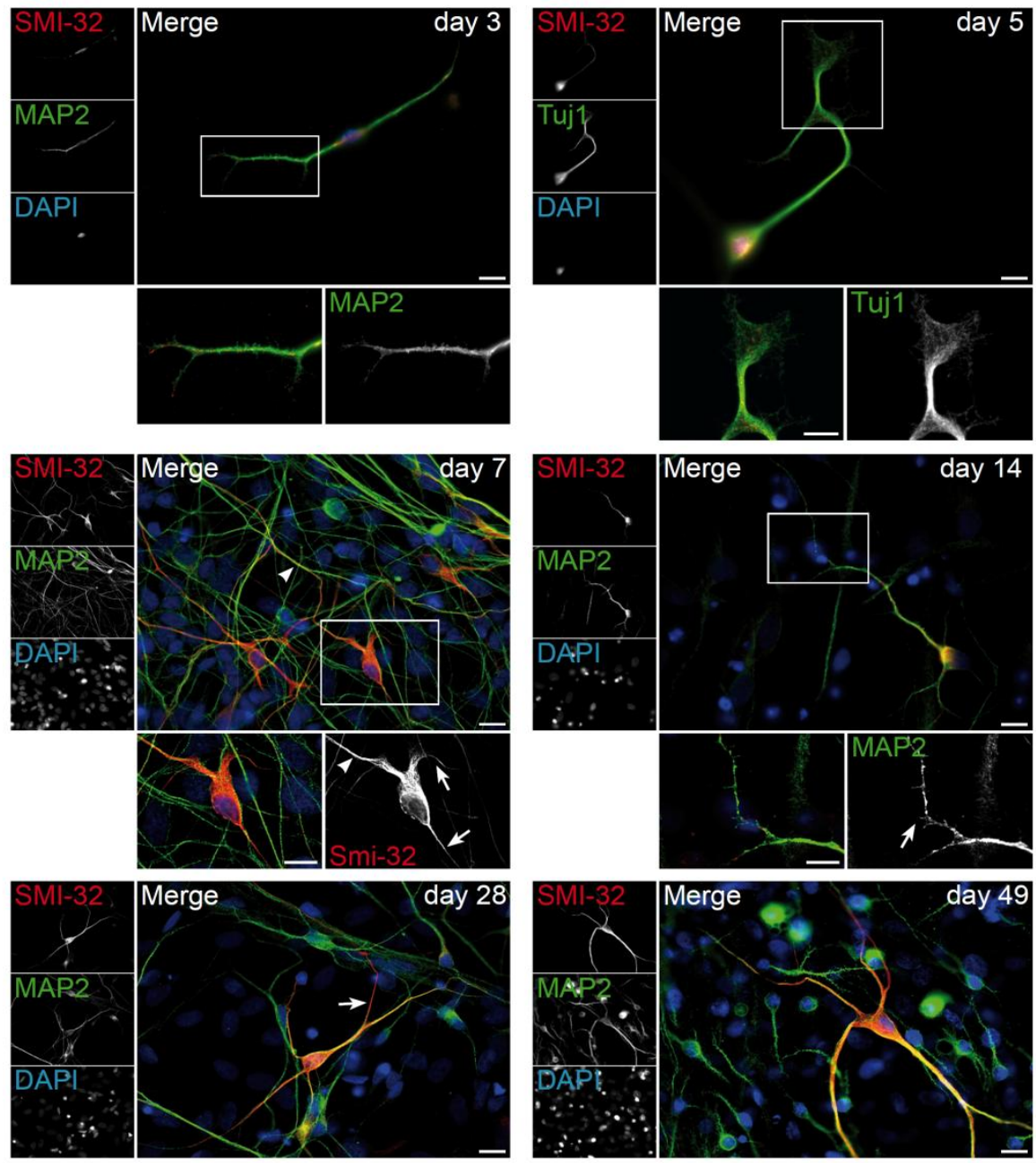

C
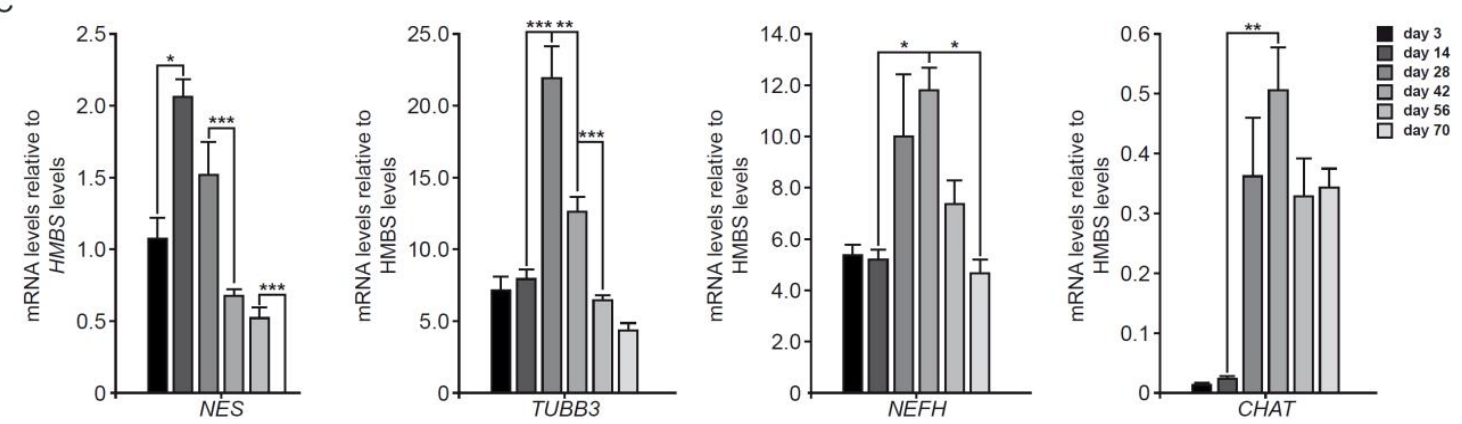

Figure 7 Time Course of the Development of hiPSCs derived Motoneurons

(A) Schematic overview of MN development. (B) On day 3-5 after final plating MNs showed a bipolar morphology with early filopodia-like structures and axonal growth cones. Neurite outgrowth started at day 7 . Usually one extremely long neurite was observed, proposed to be the developing axon. 4 weeks after plating MNs featured a long and mature axon. From day 5 onward size of the individual MNs, dendritic tree and axonal length steadily increased up to 49 days in culture. MNs were

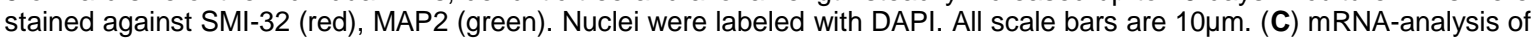
the expression level of neural proteins during MN development. ANOVA analysis of the transcript levels of the stem cell marker nestin (NES) revealed significant differences between the depicted time points ( $F$-value: 17.04; $p<0.0001)$, the neuronal marker tubulin $\beta$ III (TUBB3) (ANOVA: Fvalue: 24.23 ; $p<0.0001$ ), the motoneuron specific marker NEFH (SMI-32) (ANOVA: F-value: $5.29 ; \mathrm{p}=0.003$ ) and CHAT (Choline acetyltransferase) (ANOVA: F-value: 6.93; $\mathrm{p}=0.002$ ). Expression levels are shown relative to the housekeeping gene HMBS ( $n=4$ cells per time point). Results from post-hoc $t$-tests with Bonferroni adjustment are ${ }^{*} p<0.05,{ }^{* *} p<0.01,{ }^{* * *} p<0.001$. Error bars s.e.m. 


\section{Results}

Formation of a long and mature axon is an important step in motoneuron development. Polarization of the motoneuron started with the formation of a long single neurite featuring a growth-cone like structure and filopodia-like structures. This early axonal "progenitor" extension was elongated continuously. However, until 3 weeks of initiation of terminal differentiation, the axon and the axon hillock did not express mature axon specific markers. Upon day 20, markers which normally spare the axon such as MAP2 (microtubule associated protein 2), were still present throughout all neuronal projections. During ongoing differentiation, markers for the axonal hillock, e.g. IKK subunit IKB- $\alpha$ of NFKB [163] were expressed in the motoneurons at specific sites (Fig. 8A). In parallel, MAP2 was not longer present in the axon whereas SMI-32 still stained the mature axon (Fig. 8B). After 7 weeks, motoneurons featured a completely developed dendritic tree and a mature long axon. Measuring axonal and dendritic length thorough all steps of motoneuron development showed that the axonal and dendritic length steadily increased upon day 84 . The number of primary dendrites and branching points per dendrite, however, reached maximum values already within in the first 4 weeks after plating (Fig. 8C). 


\section{Results}
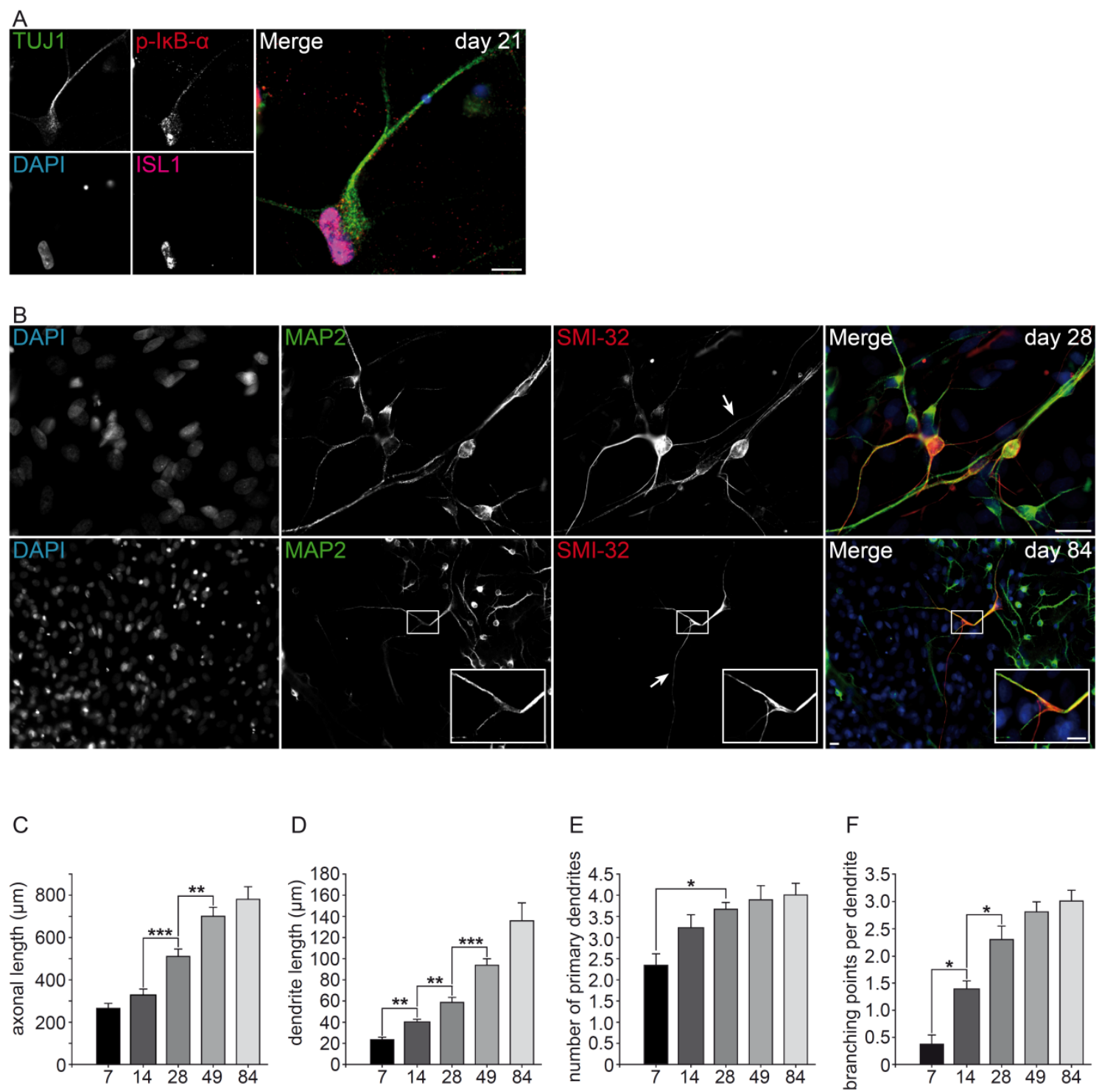

Days after final plating

\section{Figure 8 Development of a mature Axon}

(A) Formation of a defined and mature axon began 3 weeks after plating visualized by a shift of phosphorylated lkB- $\alpha$ (red) from the nucleus into the emerging axon. Staining was accomplished with TUJ1 (green) and ISL1 (magenta). (B) Double staining with MAP2 (green) and SMI-32 (red) defines a clear axonal and dendritic compartment. Majority of MNs showed a single SMI-32 positive axon after 28 days of differentiation. All scale bars are $10 \mu \mathrm{m}$ and nuclei were stained with DAPI. (CF) Measurement of axonal length (C), dendrite length (D), number of primary dendrites (E) and dendritic branching points (F) at indicated time points of differentiation. ANOVA analysis revealed significant differences between time points for axonal length ( $F$-value: 29.73; $p<0.0001$ ), dendritic length ( $F$-value: 25.76; $p<0.0001$ ), number of primary dendrites ( $F$ value: $4.43 ; p=0.001)$ and branching points (F-value: $28.27 ; p<0.0001)$. Data are mean values \pm s.e.m. $(n=10$ cells per time point). Results from post-hoc t-tests with Bonferroni adjustment are ${ }^{*} p<0.05,{ }^{* *} p<0.01,{ }^{* * *} p<0.001$. 
Results

\subsection{Development of synaptic Structures in hiPSCs derived Motoneuron Cultures}

\subsubsection{Development of the presynaptic Compartment}

To analyze the development of the presynaptic compartment we focused on two presynaptic proteins with respect to time dependent expression and localization. Synaptophysin (SYP), marking all synaptic vesicles and piccolo (PCLO), a large scaffolding protein of the presynaptic active zone, have been analyzed over a time frame of 70 days in vitro. Interestingly, SYP was found to be expressed very early during motoneuron development. Already at day 10 after plating SYP could be detected in large vesicles along the presumed early axon and was especially located in leading edge structures (day 14) (Fig. 9A). From day 14 onwards, SYP was strongly abundant along the longest motoneuron neurite, followed by a more specific localization of SYP in the presynaptic compartment at day 28 (Fig. 9C,E). SYP positive puncta increased constantly over the time peaking at day 70 (Fig. 9G). After day 70, SYP positive puncta slightly decreased indicating that SYP became more for the presynaptic side than to synaptic transport vesicles (Fig. 12A). PCLO was initially detected juxtaposed to the nucleus at day 10 (Fig. 9B) and was not located to the synaptic sites before day 28 . Though, PCLO was highly expressed in the leading edge of the presumed axon in motoneurons two weeks after plating (Fig. 9D). In contrast to SYP, PCLO positive puncta continually increased until day 70. Thus, frequent synaptic localization of PCLO was not observed before day 70 (Fig. 9H).

Transcript levels of presynaptic proteins were regulated in a slightly different manner. All four investigated presynaptic proteins SYP, PCLO, BSN (Bassoon, another large structural protein of the presynaptic active zone) and NRXN1 (neurexin 1, a presynaptic transmembrane protein) steadily increased after initiating terminal motoneuron differentiation, peaked at day 28 , followed by a decrease upon day 70. SYP showed a strong increase between day 3, day 14 and day 28 , whereas $P C L O$ made its steepest increase from day 14 to day 28 . BSN and NRXN1 transcript levels displayed comparable expression patterns (Fig. 9I). 


\section{Results}
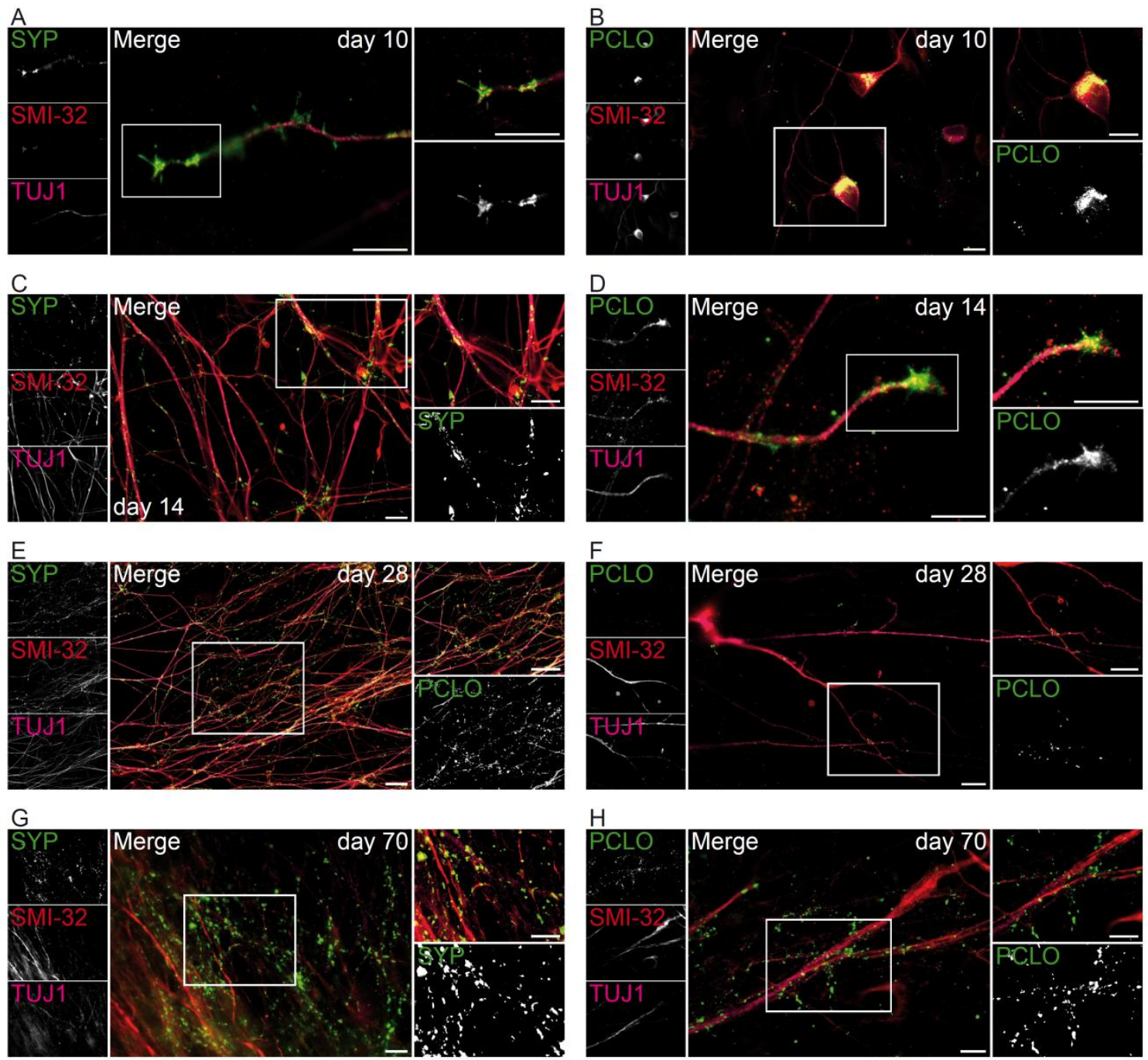

I
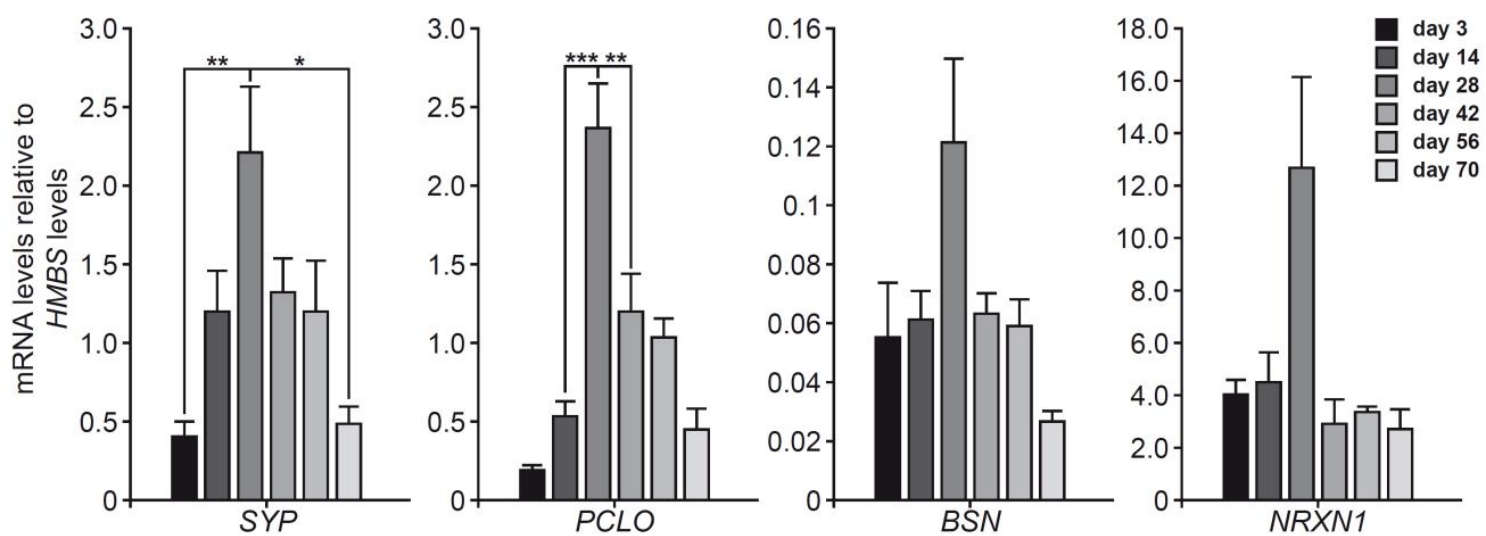

Figure 9 Development of the Presynapse

(A) After 10 days in culture synaptophysin (SYP, green) was found in small axonal protrusions representing filopodia-like structures and in axonal growth cones. (B) In contrast to SYP, piccolo positive (PCLO, green) structures were solely detected in a distinct perinuclear area. (C) From day 14 onward SYP positive vesicles were present along the neural projections. (D) PLCO positive signals were highly abundant in axonal growth cones. (E, G) According to the stage of differentiation, SYP positive vesicles highly increased from day 14 to day 70 . $(\mathbf{F}, \mathbf{H})$ PCLO positive vesicles were present after 4 weeks in culture and number increased up to 10 weeks of differentiation. SMI-32 (red) and TUJ1 (magenta). All scale bars are $10 \mu \mathrm{m}$. (I) Regulation of mRNA-levels of presynaptic proteins: synaptophysin (SYP), piccolo (PCLO), bassoon $(B S N)$ and neurexin1 (NRXN1) during development. ANOVA analysis revealed significant differences between time points for SYP (F-value: 5.04; $\mathrm{p}=0.006$ ), PCLO (F-value: 15.20; $\mathrm{p}<0.0001), N R X N$ (F-value: 4.38; $\mathrm{p}=0.013$ ) and $B S N$ (F-value: $6.12 ; p=0.003$ ). Expression levels are shown relative to the housekeeping gene HMBS ( $n=4$ independent experiments, 10 cells per time point). Results from post-hoc t-tests with Bonferroni adjustment are ${ }^{*} p<0.05,{ }^{* *} p<0.01,{ }^{* * *} p<0.001$. 


\subsubsection{Development of the postsynaptic Compartment}

The postsynaptic compartment of excitatory synapses is characterized by the formation of the dendritic spine and the postsynaptic density (PSD). The PSD, a dense network underneath the postsynaptic membrane, is composed of a larger number of proteins including anchoring molecules, receptors and ion channels and its specification and maturation is assumed to be initiated and developed in parallel to the presynaptic specialization [62]. In deeper layers of the PSD, large scaffolding proteins of the ProSAP/SHANK family are localized [107]. HOMER1, a binding partner of the ProSAP/SHANK family members, which is responsible for crosslinking proteins of the PSD, was already traceable at day 14 and HOMER1 positive puncta could be found within the dendritic compartment (Fig. 10A). According to ongoing differentiation, synaptic localization of HOMER1 increased over time. After 7 weeks, HOMER1 was detectable in dendritic spines and three weeks later HOMER1 positive synapses were found frequently in the motoneuron cultures (Fig. 10C,E). ProSAP1/SHANK2 is known to be among the first molecules of the PSD [67]. In general, timeframe and expression of ProSAP1 was found to be comparable to HOMER1 (Fig. 10B-F).

These observations were further underlined by a comparable mRNA level expression at the related time points of differentiation. Transcript levels of both markers peaked sharply after 4 weeks of differentiation followed by a continuous decrease. mRNA levels of DLG4 (transcript name of PSD 95), a well-known scaffolding protein of the PSD [74], also peaked on day 28 but remained on a relatively high level until late time points with a second peak on day 56 . ProSAP3/SHANK1, which is essential for synapse maturation on rat hippocampal neurons [68] reached the highest mRNA levels also 4 weeks after final plating with a consistent expression until day 42 followed by a slight decrease at day 70 . mRNA levels of Neuroligin (NLGN1), a postsynaptic adhesion protein of excitatory synapses [181], showed only a mild downregulation after its peak on day 28. Similar to DLG4, mRNA transcription of NLGN1 remained on a relatively constant level (Fig. 10G). 


\section{Results}

A

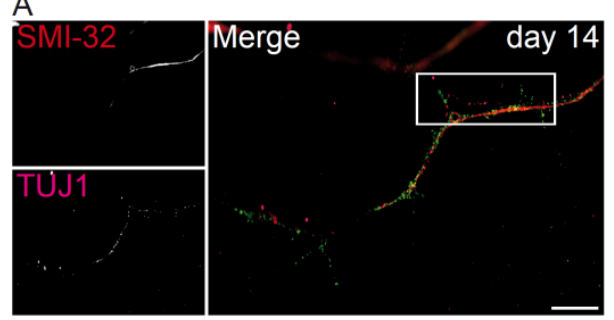

C

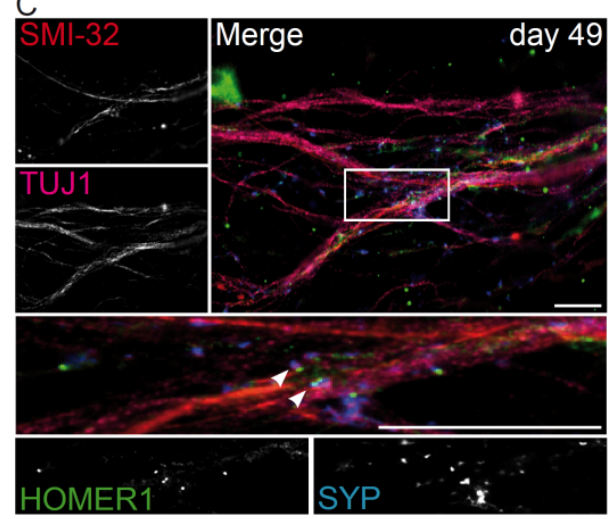

E

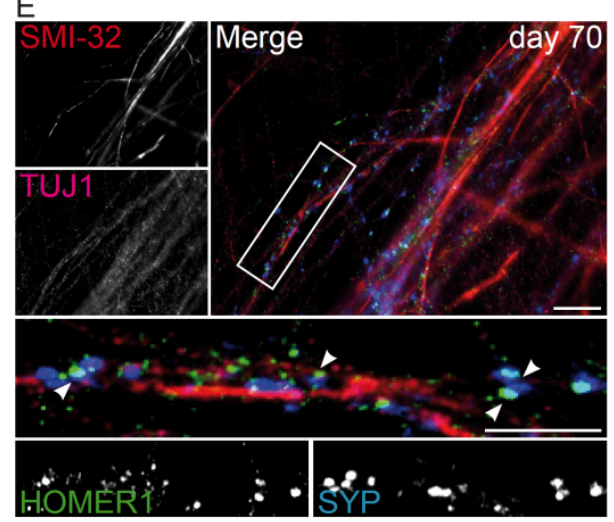

B

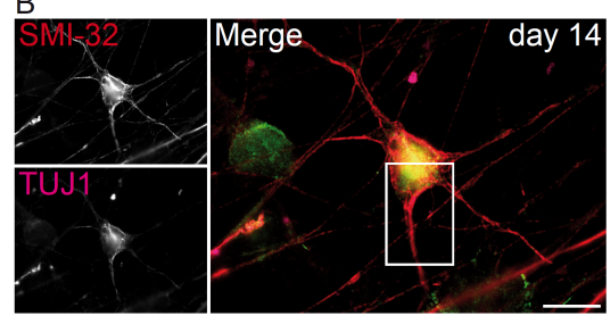

D

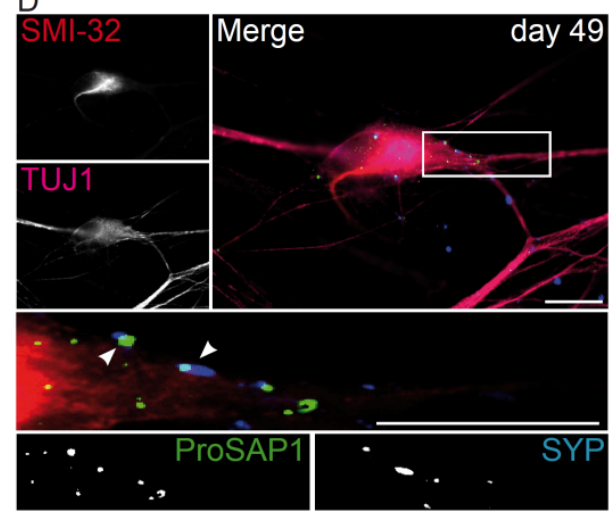

F

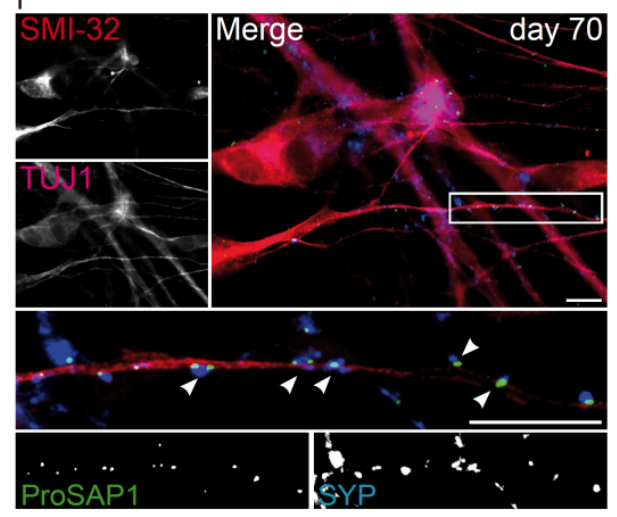

G
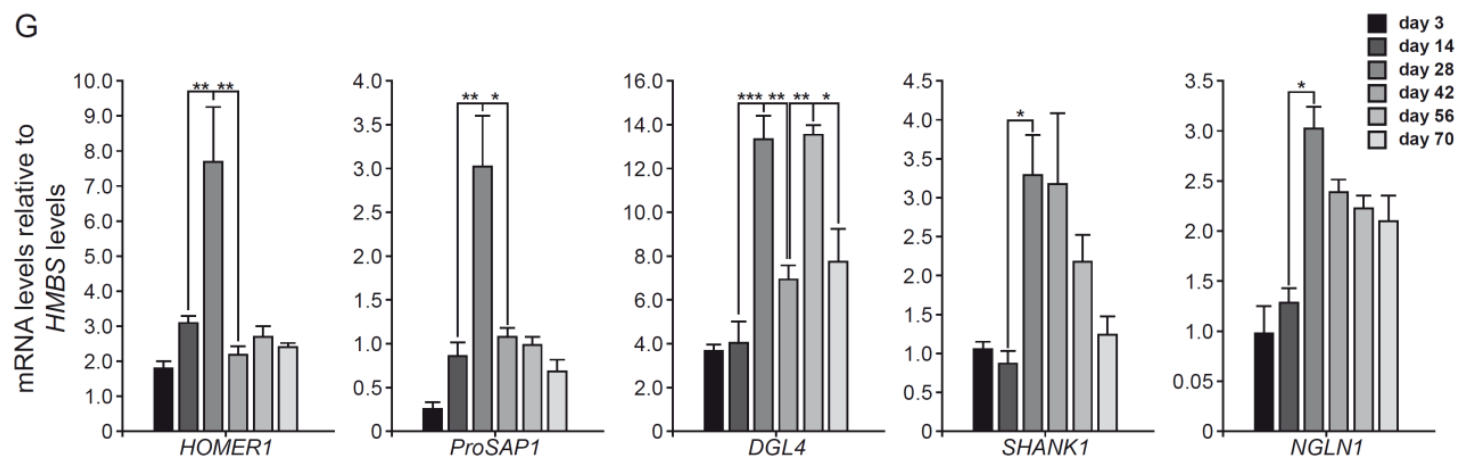

Figure 10 Development of the Postsynapse

(A, B) 14 days after plating the postsynaptic protein HOMER1 (green) was evenly distributed within the neurites of the differentiating MNs, whereas ProSAP1/SHANK2 (green) was mainly allocated in the cytoplasm. (C) First axo-dendritic contacts emerged after 7 weeks in culture. The juxtaposed signal of SYP (blue) and HOMER1 represents an early developing synaptic contact. (D) Synaptic localization of ProSAP1/SHANK2 was traceable after 49 days in culture. (E, F) Colocalization of SYP (blue) and postsynaptic proteins (HOMER1 / ProSAP1, green) could frequently be detected at later time points of differentiation. All scale bars are $10 \mu \mathrm{m}$. (G) Regulation of transcript levels of typical postsynaptic proteins like HOMER1, DLG4 and ProSAP1/SHANK2, ProSAP3/SHANK1 and for neuroligin1 (NLGN1), an adhesion protein of the excitatory postsynapse. ANOVA analysis revealed significant differences between time points for HOMER1 (Fvalue: 7.81; $\mathrm{p}<0.0001$ ), ProSAP1/SHANK2 (F-value: 9.69; $\mathrm{p}<0.0001$ ), DGL4 (F-value: 18.17; $\mathrm{p}<0.0001$ ), ProSAP3/SHANK1 (F-value: 4.07; $\mathrm{p}=0.015$ ) and NGLN1 (F-value: 11.20; $\mathrm{p}<0.0001$ ). Expression levels are shown relative to the housekeeping gene HMBS ( $\mathrm{n}=4$ independent experiments, 10 cells per time point). Results from post-hoc t-tests with Bonferroni adjustment are ${ }^{*} p<0.05,{ }^{* *} p<0.01,{ }^{* * *} p<0.001$. 


\subsection{Formation of mature Synapses in hiPSCs derived Motoneuron Cultures}

\subsubsection{Immunoflourescence Analysis of mature Synapses}

To analyze synaptogenesis double stainings against pre- (PCLO / SYP) and postsynaptic proteins (HOMER1) were performed at two different time points of motoneuron development (Fig. 11A,B). In immunofluorescence imaging, the juxtaposed position of pre- and postsynaptic proteins, characterized by a small area of overlapping colors, is specific for excitatory synapses. At early time points of differentiation when synaptic proteins are built and transported along the neuronal projections, overlapping structures could rarely be observed within the first 5 weeks after plating. According to ongoing development and differentiation, a specific colocalization at maturing synaptic contacts was visible. The first specific colocalization of both proteins was traceable after 6 weeks of differentiation and constantly increased up to day 84 (Fig. 11A,B).

\subsubsection{Ultrastructural Analysis of mature Synapses}

Further ultrastructural investigations using electron microscopy of synaptic development during motoneuron differentiation was performed. At early stages of development large vesicles, which had already been described for immature hippocampal neurons, and seemed to be bigger in size and more unevenly formed than mature synaptic vesicles (SCV), were found along the neural projections [67] (Fig. 11C, d28). Accumulation of these large, clear vesicles was often found at premature contact zones between neurons (Fig. 11C, d35). At about 6 weeks of differentiation, small clear vesicles (SCV) typical for neurotransmitter filled vesicles appeared. Furthermore, dense core vesicles (DCV) were detectable in the presynaptic compartment. Axonal organelles, e.g. mitochondria were increasingly abundant in the axon at this time point (Fig. 11C, d42). After 7 weeks in culture, first, early synaptic contacts were observed. At later stages in vitro (10 and 12 weeks), clear polarized synaptic contacts were formed. A mature active zone (AZ) featuring a large pool of synaptic vesicles, docked vesicles and less dense core vesicles were visible at the presynaptic side. The postsynaptic specification is characterized by the development of the electron dense PSD, underneath the postsynaptic membrane, apparent 70 and 80 days after plating, respectively (Fig. 11C, d70 and d84). 


\section{Results}

A
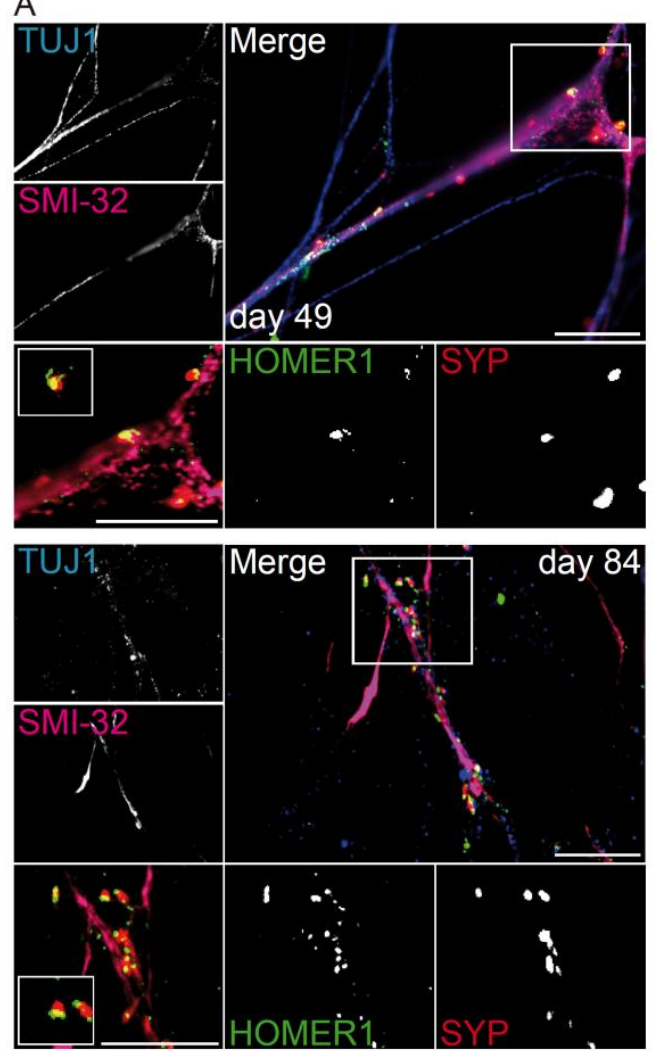

B
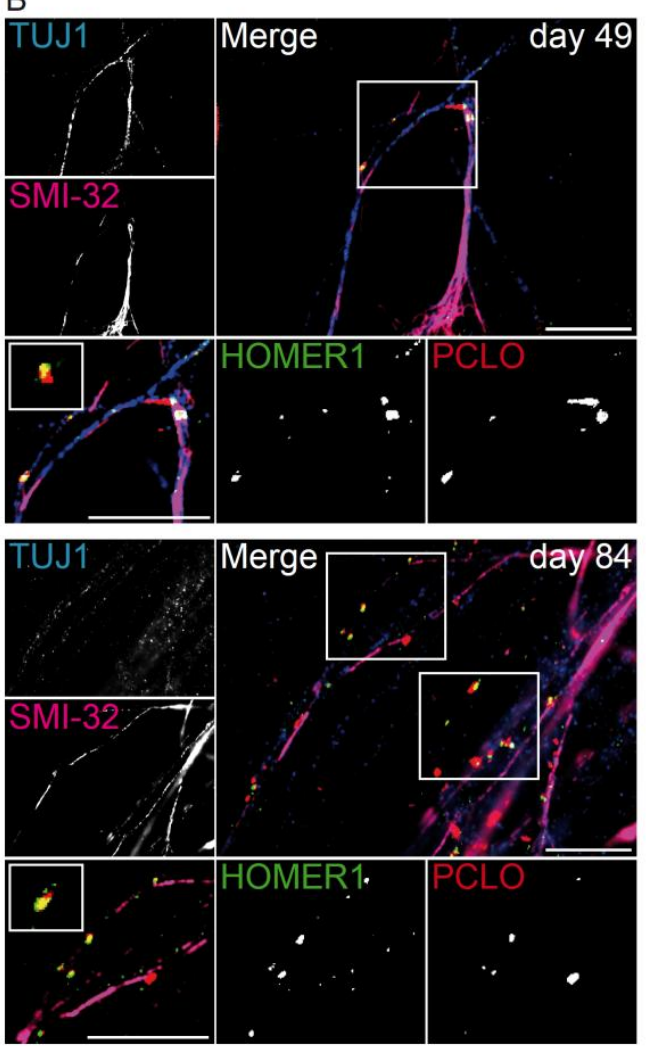

C

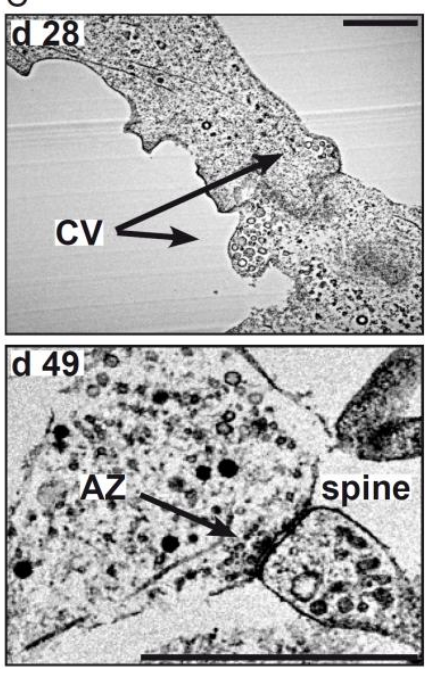

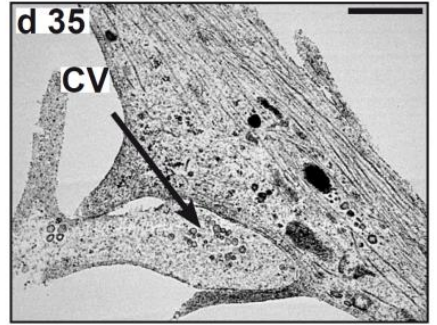

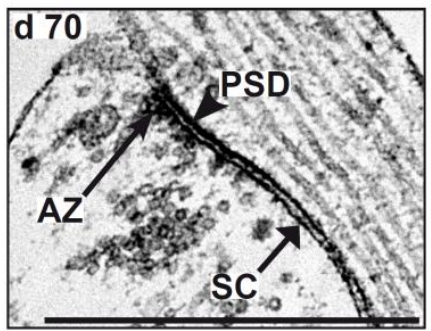

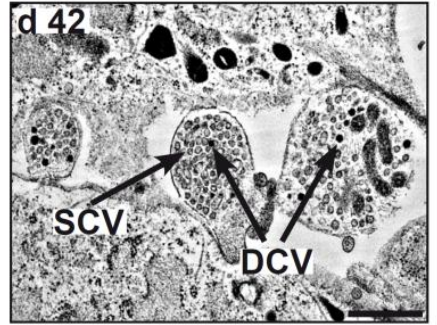

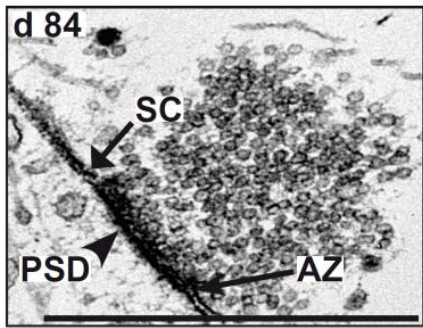

Figure 11 Characterization of Developing Synapses

(A-B) To analyze synaptic progression, double staining against presynaptic proteins SYP / PCLO (red) and the postsynaptic protein HOMER1 (green) had been performed at two different time points of synaptic development. Colocalization of SYP (A) or PCLO (B) with HOMER1 denotes the development of mature excitatory synapses. All scale bars are $10 \mu \mathrm{m}$. (C) Ultrastructural investigation of the synaptic development was accomplished by transmission electron microscopy. At early time points of synaptogenesis (week 4 of differentiation) filopodia-like structures were filled with large clear vesicles (CV) (C, day 28). Axo-dendritic contacts seemed to induce accumulation of large vesicles at the axonal department (C, day 35$)$. From week 6 onward, dense core vesicles (DCV) as well as transmitter filled vesicles (SCV) were often observed in axonal protrusions close to the dendritic compartment (C, day 42 ). First early synaptic contacts emerged 49 days after final plating (C, day 49). At day 70 and 84 excitatory synapses were detected, demonstrating a typical polarized morphology with an active zone (AZ) and a postsynaptic density (PSD). Both compartments were separated by a synaptic cleft (SC) (C, day 70 and 84$)$. All scale bars are $1 \mu \mathrm{m}$. 


\subsubsection{Quantitative Analysis of mature Synapses}

Quantitative analyses of pre- and postsynaptic protein expression demonstrated that SYP positive puncta appeared earlier but during continual progressive synaptogenesis the total amount of SYP and PCLO positive puncta converged (Fig. 12A). Colocalizing signals of SYP / HOMER1 and PCLO / HOMER1 steadily increased from day 49 onwards revealing ongoing synapse formation and maturation. We found about 4-5 SYP / HOMER1 and 3 PCLO / HOMER1 signals per $30 \mu \mathrm{m}$ dendritic length after 84 days in culture (Fig. 12B).

\subsubsection{Electrophysiological Analysis of mature Synapses}

Additionally, hiPSCs derived motoneurons showed spontaneous active currents after 7 weeks in culture. These currents were predominantly inwardly directed currents, which could be fitted by monoexponential functions. The mean decay time constant was $2.39 \pm 0.56 \mathrm{~ms}$ ( $\mathrm{n}=17$ events) (Fig. 12C). Further analysis revealed that synaptic currents could reversibly be suppressed by CNQX, an AMPA receptor antagonist, indicating the existence of functionally operating glutamatergic synapses within these cell cultures (Fig. 12D, right panel). These findings fit closely into the current theory that interneuronal synapses formed by motoneurons are mainly based on glutamatergic signaling [226]. In contrast to these observations, synaptic currents could not be blocked by the application of AP-5, an NMDA receptor agonist (Fig. 12D, left panel). We hypothesize that the development of NMDA receptors may either occur later in embryonic development or delayed in vitro compared to AMPA receptors in human motoneurons. 


\section{Results}

A

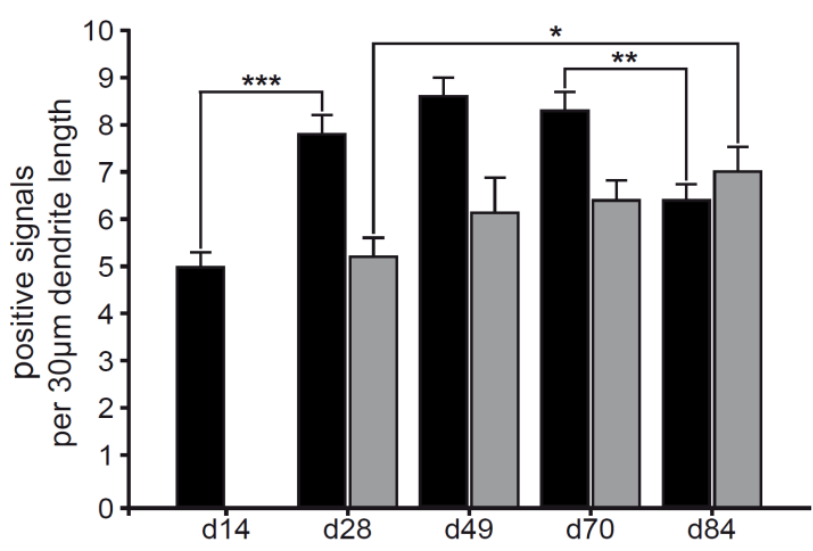

SYP
B

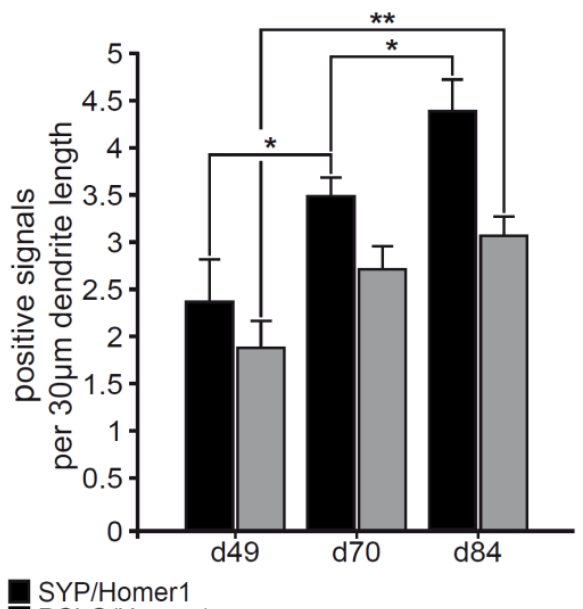

SYP/Homer1

C
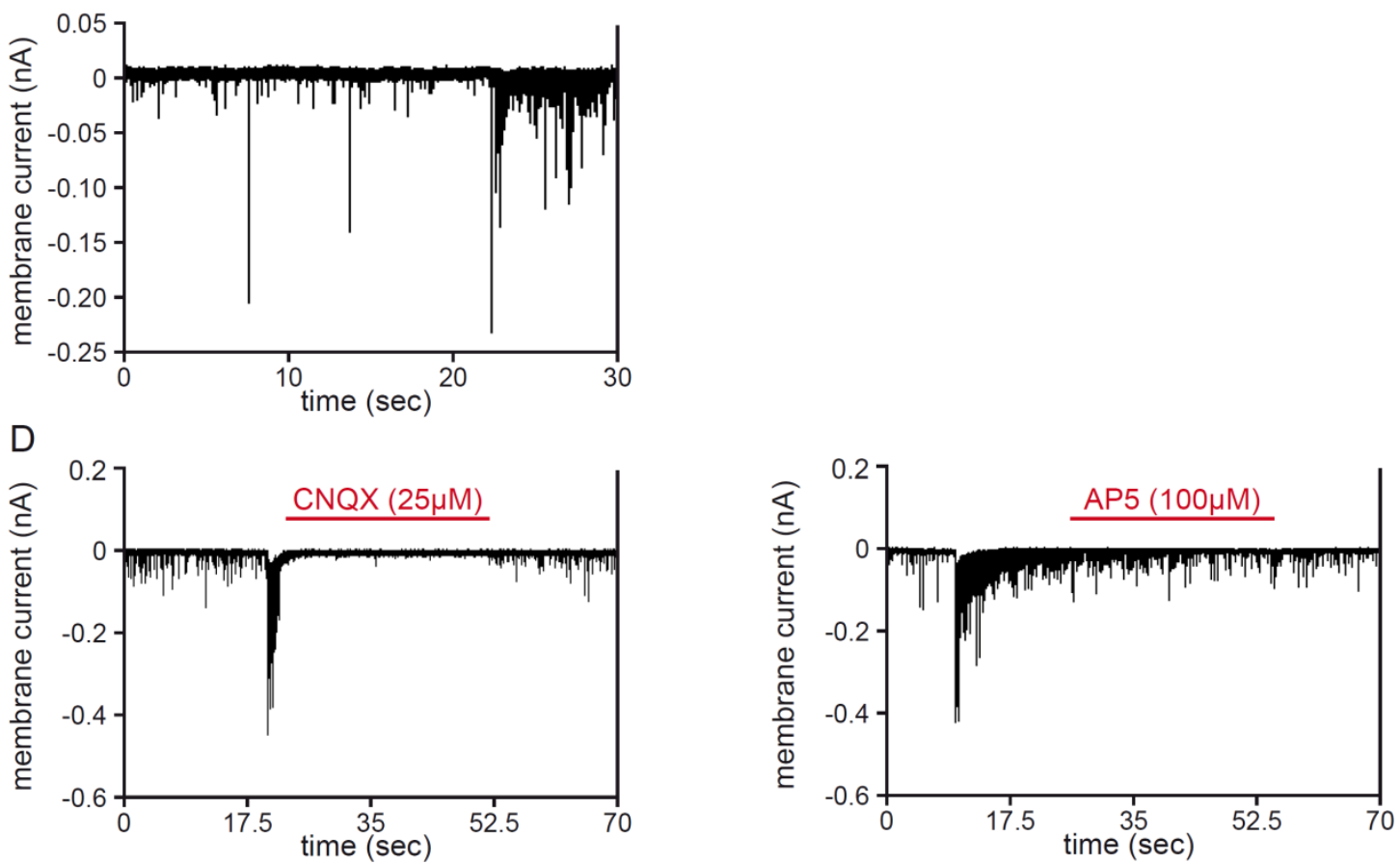

Figure 12 Quantification of Developing Synapses

$(\mathbf{A}, \mathbf{B})$ For quantitative analysis of synapse number, SYP / PCLO positive signals and the colocalization of SYP / HOMER1 and PCLO / HOMER1 spots were counted along a defined dendritic length $(30 \mu \mathrm{m} ;$ mean \pm s.e.m.; $n=20)$. ANOVA analysis revealed significant differences between time points for SYP positive signals ( $F$-value: 15.92; $p<0.0001$ ), SYP / HOMER1 positive signals ( $F$-value: 11.84; $p<0.0001$ ) and for PCLO / HOMER1 positive signals ( $F$-value: $4.73 ; p=0.013$ ), but not for PCLO positive signals (F-value: $2.45 ; \mathrm{p}=0.071$ ). Results from post-hoc t-tests with Bonferroni adjustment are ${ }^{*} \mathrm{p}<0.05$, ${ }^{* *} p<0.01,{ }^{* * *} p<0.001$. Note, that there was a significant increase of the number of SYP positive vesicles from 14 days to 28 days of differentiation followed by a slight decrease at day 84 indicating, that SYP signals became more specific for the presynaptic active zone and less for transport vesicles. Colocalizing signals increased during development $(n=4$ independent experiments, 20 cells per time point). (C) Spontaneous excitatory synaptic currents in 35-day-old motoneuron cultures. The mean decay time constant was $2.39 \pm 0.56 \mathrm{~ms}$ ( $\mathrm{n}=17$ events). (D) Reversible suppression of the excitatory synaptic currents by $25 \mu \mathrm{M}$ CNQX (reversible inhibitor of the AMPA receptor). Contrary to that, synaptic currents cannot be blocked by AP-5 (reversible inhibitor of the NMDA receptor, $100 \mu \mathrm{M}$ ) suggesting, that AMPA receptors develop earlier than NMDA receptors. 


\section{Results}

\subsection{Generation of functional neuromuscular Junctions}

\subsubsection{Differentiation of primary and human Myoblasts}

To investigate functionality hiPSCs derived motoneuron NMjs, cells were cocultured with primary mouse myotubes as well as human myotubes derived from muscle biopsies. Mouse and human myoblasts were initially differentiated until they featured elongated myofibrils and expressed muscular specific markers like $\alpha$-actinin and $\alpha-M H C$ (myosin heavy chain) (Fig. 13A). Differentiation of mouse myoblasts turned out to be more than twice as fast in differentiation compared to human myoblasts. Rare spontaneous contractions of primary myotubes were observed 10 days after inducing myoblast differentiation. However, spontaneous contractions could not be detected in human myotubes cultures.

\subsubsection{Co-culture of Myotubes and Motoneurons}

Motoneurons (at day 28 of the differentiation protocol) were (co-) cultured alone or with 14-day-old primary murine muscle cells (Fig. 13 A,B). Cells were analyzed after two weeks of co-culture.

To visualize nerve muscle connections fluorophore labeled $\alpha$-bungarotoxin ( $\alpha$ BTX) is a bona fide tool to detect motor end plates in vitro as it binds very specific to the acetylcholine receptor (AChR). Tagged a-BTX was applied to the co-culture system and we observed accumulation of the AChR indicating the formation of neuromuscular junctions (NMjs), whereas pure myotube cultures did not bind $\alpha$ BTX at specific sites (Fig. 13 A,B). Confocal microscopy demonstrated $\alpha$-BTX positive structures marking the postsynaptic side of the myoblast (visualized by $\alpha$ $\mathrm{MHC}$ ) very close to motoneuron axons filled with SYP positive synaptic vesicles (Fig. 13 C,D). 


\section{Results}

A

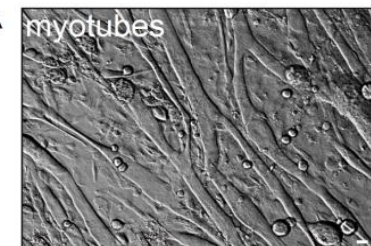

B

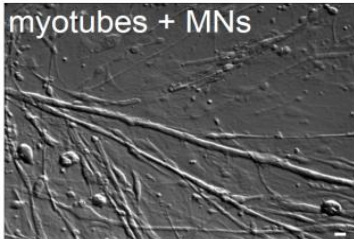

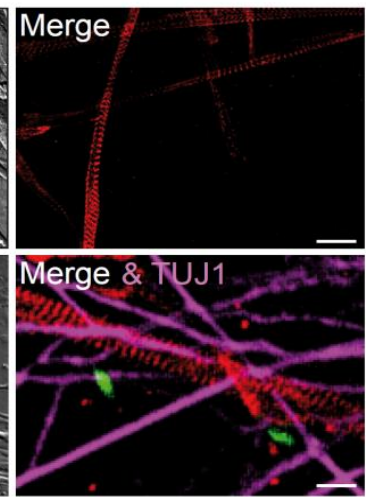
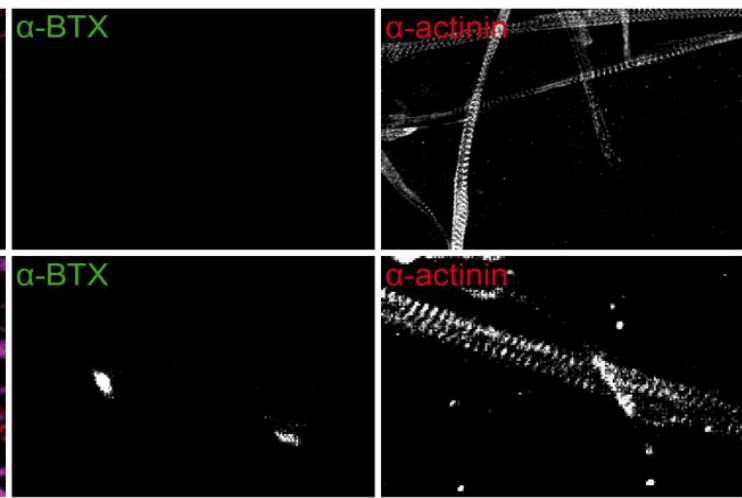

C
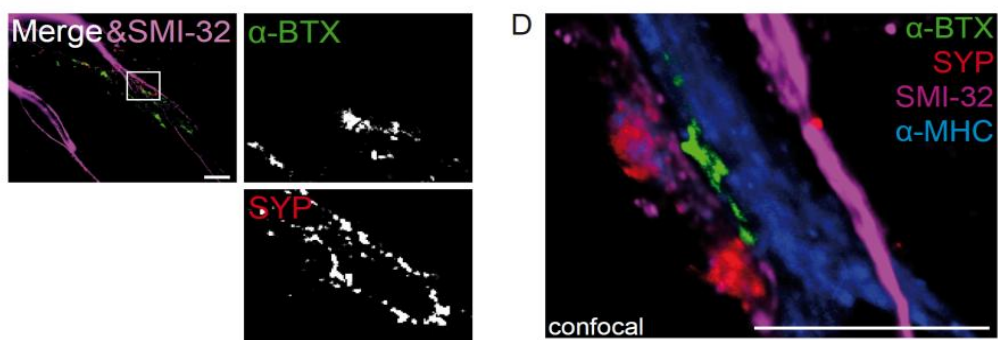

E
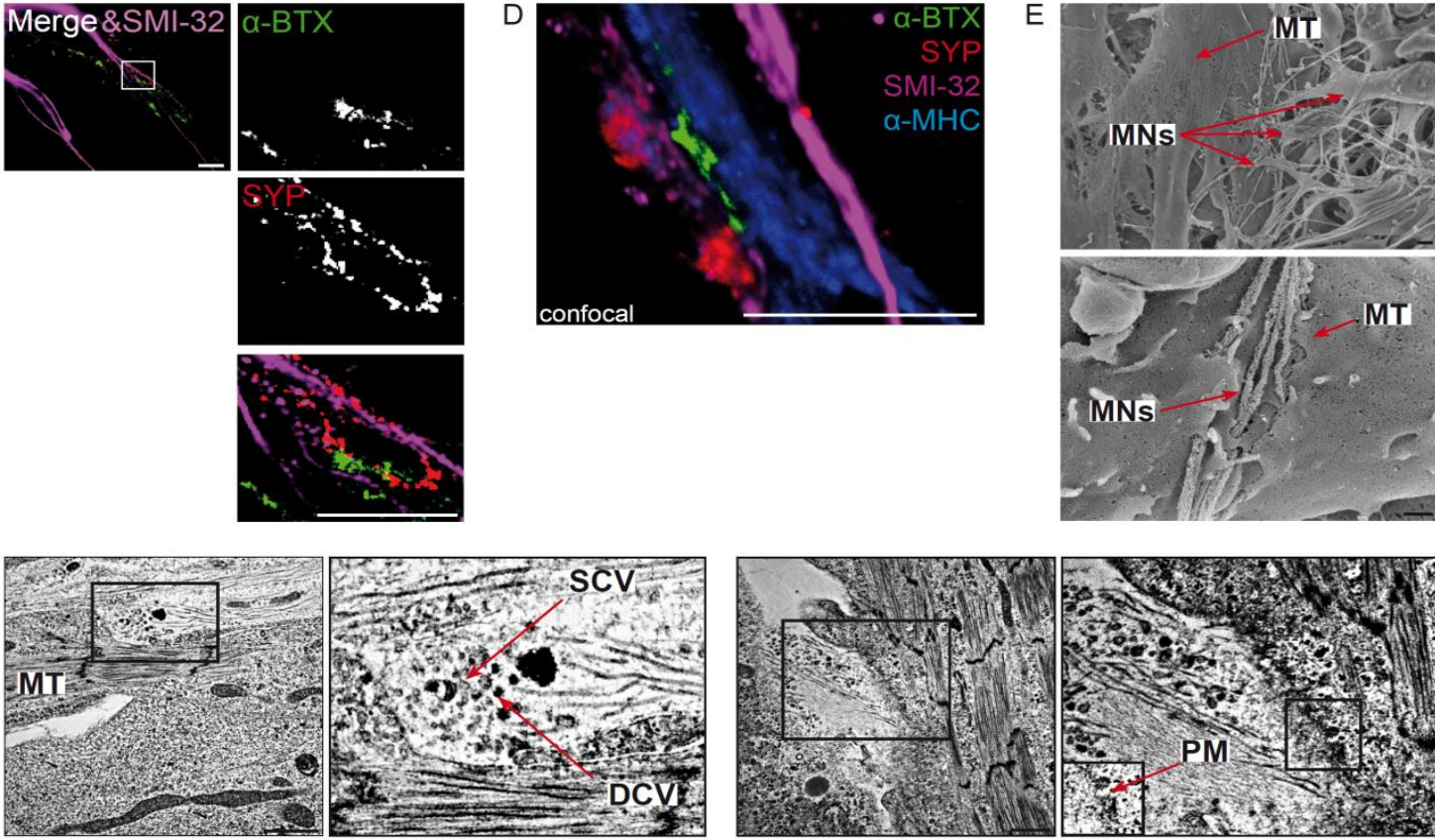

G Merge
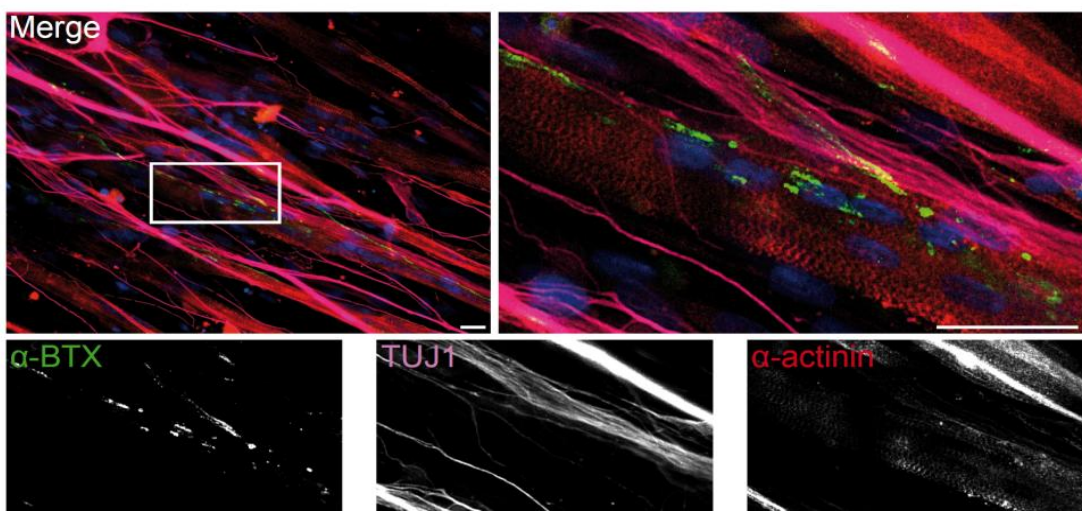

Figure 13 Co-culture of hiPSCs derived Motoneurons and Myotubes

Primary mouse myotubes (MTs) had been cultured alone or co-cultured with hiPSCs derived motoneurons (MNs). Acetylcholine receptors $(A C h R)$ were visualized by fluorescence-labeled $\alpha$-bungarotoxin ( $\alpha$-BTX, green). (A) Bright-field microscopy images of MTs showing their typical elongated fibers. MTs expressed a-actinin (red) (A,B) On day 29 of the differentiation protocol, MN-progenitors (TUJ1, magenta) were plated onto a monolayer of MTs. Explicit AChR accumulation ( $\alpha$-BTX, green) was observed after two weeks of co-culture. In MT single culture AChR accumulation could not be detected. (C) SYP positive vesicles (red) juxtaposed to an AChR-enriched region demonstrated the formation of neuromuscular junctions (NMJs). (D) Clustering of the AChR along the MT membrane was shown by confocal analysis. (E) SEM showing an overview (left side) and the detailed morphology (right side) of MN and MT co-culture. (F) TEM of MTs co-cultured with MNs. The MTs were clearly identified by their obvious cross-striation pattern. Adjacent to the MTs, axonal terminals were detected, filled with small and organized synaptic vesicles (SV) and dense core vesicles (DCV) (arrows). The beginning infolding of the postsynaptic membrane (PM) was observed. All scale bars are $1 \mu \mathrm{m}$. (G) MNs were also co-cultured with human MTs derived from muscle biopsies. According to the findings of co-culture experiments with mouse myotubes an obvious accumulation of the AChR was visible. Scale bars in bright-field imagines images are $20 \mu \mathrm{m}$; all other scale bars are $10 \mu \mathrm{m}$. 
Scanning electron microscopy provided an additional insight in the morphology of motoneurons co-cultured with myotubes. Neural projections seemed to be embedded in the assumed myotubes sarcolemma (Fig. 13E).

On the ultrastructural level, well differentiated myotubes were easily identified by a clear striation of myofibrils (MF). Crucial features of functional neuromuscular connections such as the infolding of the postsynaptic membrane (PM) in the opposite to axonal terminals filled with synaptic vesicles (SCV) and dense core vesicles (DCV) were detected (Fig. 13F).

HiPSCs derived motoneurons were additionally co-cultured with human myotubes derived from muscle biopsies. Accumulation of the AChR was observed in a comparable fashion to primary murine myotubes but with lower incidence (Fig. 13G).

\subsubsection{Functional Analyses of neuromuscular Junctions between human Motoneurons and murine Myotubes}

Further analyses of the functional interplay between human motoneurons and murine myotubes revealed that co-cultivated muscle cells showed larger areas of concerted and more frequently contracting myotubes compared to pure myotube cultures. Contractions of human myotubes (co-) cultured alone or with MNs could not be observed after two weeks of co-culture most probably because human myotubes might need longer to obtain the same stage of differentiation and functionality as murine myotubes. In pure murine myotube cultures only few cells were spontaneously contracting.

Based on these observations, NMjs were asked to be responsible for these differences. Subsequently, inhibition of both synaptic sides of the NMjs was performed to answer this question (Fig. 14A).

First, the presynaptic transmitter release was blocked by the application of the bacterial toxin botulinumtoxin A which proteolyses the SNAP-25 unit of the SNARE complex at the NMjs. This subsequently prevents vesicles from anchoring to the membrane [9]. Application of botulinumtoxin A in the co-cultures led to a nearly complete inhibition of muscle cell contraction within $90 \mathrm{~s}$ lasting several minutes (Fig. 14B). The successful inhibition of muscle contraction by blocking acetylcholine receptors at the postsynaptic side with curare (Pancuronium) within 


\section{Results}

30 s finally underlined the maturity and functionality of synaptic communication between the motoneurons and the myotubes (Fig. 14C). Spontaneous contractions in control myotube cultures were not affected by these substances (data not shown).

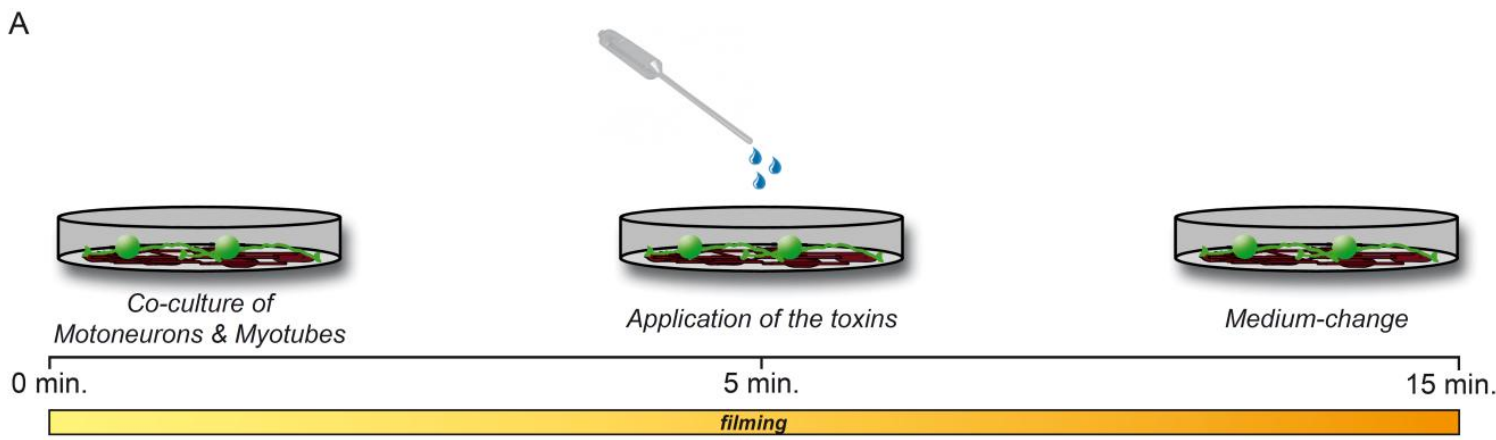

B
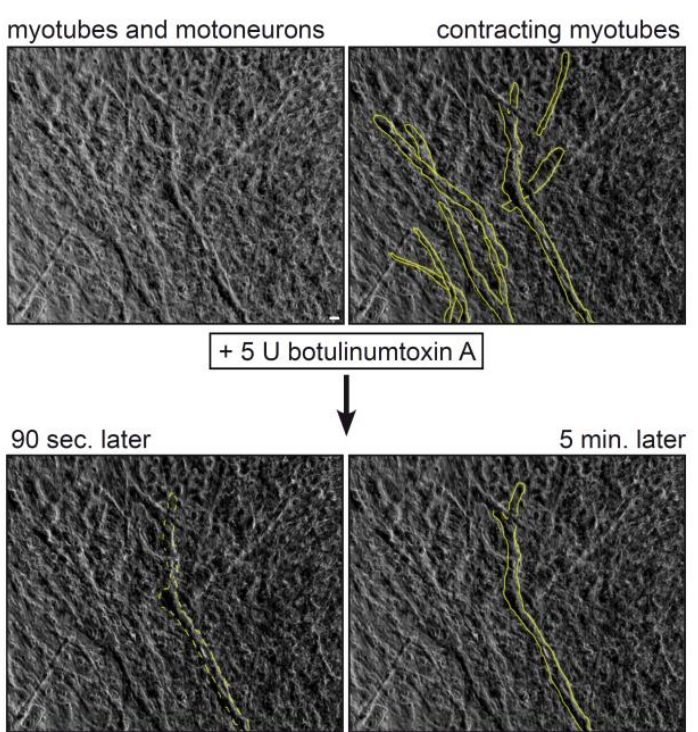

C
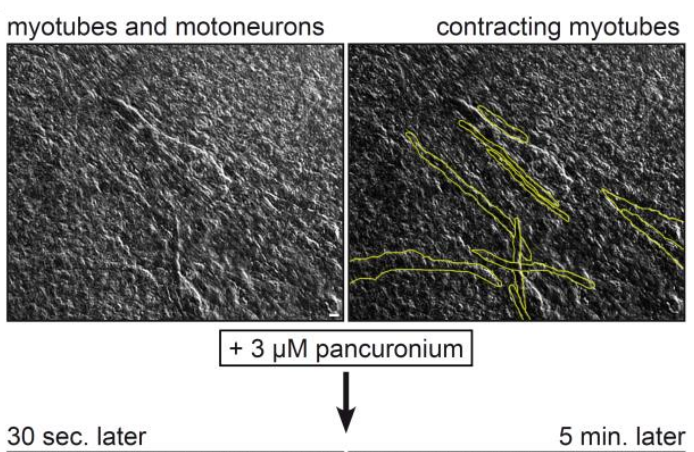

Figure 14 Functionality of neuromuscular Junctions

(A) Functional abilities of neuromuscular contacts had been investigated using 14 -day -old co-cultures. Functional active NMjs were inhibited by applying pancuronium (post-synaptic AChR inhibitor) or botulinumtoxin A (SNARE complex inhibitor) to the co-culture system. Contracting myotubes (yellow) were identified shortly before application of the toxins. (B) To inhibit acetycholine transmitter release 5 Units / $\mathrm{ml}$ of botulinumtoxin A were added to the co-culture. After $90 \mathrm{sec}$. majority of the myotubes stopped contracting and frequency remained diminished for approximately 5 minutes. (C) Briefly after application of $3 \mu \mathrm{m}$ pancuronium myotube contraction decreased strongly. Five minutes after toxin application myotubes gradually started contraction again.

\subsection{Development of primary rodent Motoneurons}

Hallmarks of neurogenesis and synaptic maturation of human iPS cell derived motoneurons were compared with the development of primary rodent motoneurons. Primary motoneurons were obtained from E14.5 old rat embryos 
and were kept up to 4 weeks in culture. Primary motoneuron cultures contained around $70 \%$ SMI-32 positive cells.

\subsubsection{Maturation of primary motoneurons}

At day 3 in vitro (DIV) motoneurons already expressed the neuronal marker (MAP2) and the motoneuron specific marker (SMI-32). During the first 3 days, most primary motoneurons formed few short dendrites and one long dominant neurite. The early presumed axon was still positive for MAP2 and SMI-32. The axon extended rapidly and only two days later (DIV 5), the motoneuron axon became negative for MAP2 suggesting a clear organization in an axonal and dendritic compartment. During the following weeks (up to DIV 20), axonal and dendritic length further increased. Additional neurites were formed and secondary/tertiary dendrites were branching out, forming a completely developed dendritic tree (DIV 20) (Fig. 15A,B). Mature primary motoneurons were further positive for acetylcholine transferase (CHAT) on DIV 20 (Fig. 15C)

On mRNA levels, transcripts specific for neuronal development (TUBB3) presented their highest levels subsequently after plating (DIV 1) and sharply increased up to DIV 14. The motoneuron specific marker peaked 2 days later (DIV 3) and gradually increased up to DIV 7, followed by a sharp reduction until DIV 14 (Fig. 15D). Furthermore, primary motoneurons were co-cultured with primary myotubes. Already 5 days after of co-culture, accumulation of the AChR was detectable. Clustering of the AChR was usually observed close axon terminal filled with synaptophysin positive vesicles (Fig. 15E). 


\section{Results}
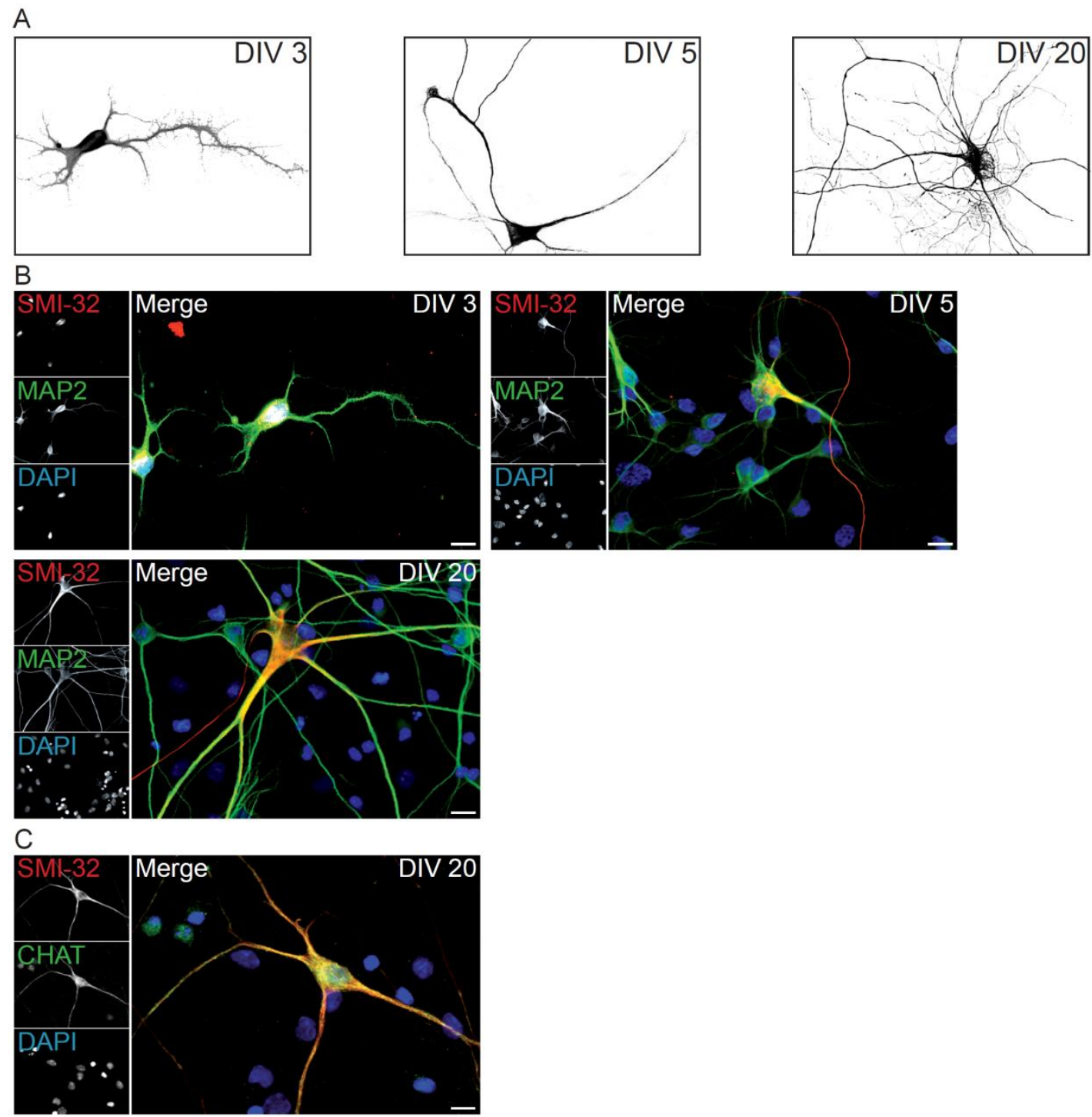

D

$\mathrm{E}$
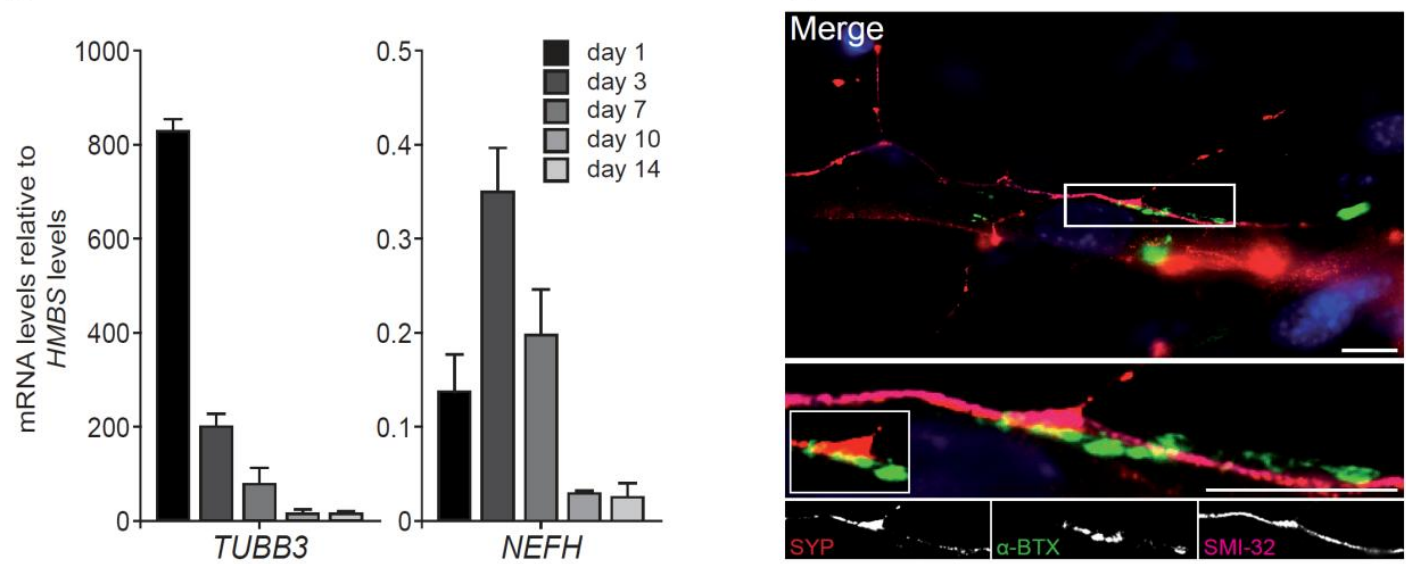

Figure 15 Time course of the Development of rat Motoneurons

(A) Schematic overview of rodent MN development. (B) On day 3 in vitro (DIV 3) MNs featured some small dendrites and an early axon with filopodia-like structures. Two days later a single SMI-32 positive mature axon was observed. From day 5 onward size of the individual MNs, dendritic and axonal length continually increased up to 20 days in culture. MNs were stained against SMI-32 (red), MAP2 (green). (C) Mature primary motoneurons further expressed acetylcholine transferase (CHAT, green), an essential enzyme for ACh biosynthesis. (D) Regulation of mRNA levels of tubulin BIII (TUBB3) and SMI$32(N E F H)$. Expression levels are shown relative to the housekeeping gene HMBS ( $\mathrm{n}=4$ cells per time point). Error bars s.e.m. (E) Rat motoneurons (SMI-32, magenta) induced accumulation of the AChR ( $\alpha$-BTX, green) when co-cultured together with primary mouse myotubes for 5 days. SYP positive vesicles (red) next to obvious AChR accumulation revealed the generation of NMJs. Nuclei were labeled with DAPI. All scale bars are $10 \mu \mathrm{m}$. 


\section{Results}

\subsubsection{Synaptogenesis of primary Motoneurons}

Synaptophysin, marking all synaptic vesicles, was traceable as soon as after 3 days in vitro. On DIV 3 SYP was mainly abundant in early axonal protrusions. After one week in culture SYP was increasingly organized at the presynaptic compartment. The number of SYP positive vesicles constantly increased until 20 days in culture (Fig. $16, \mathbf{B})$.

In accordance to the presynaptic maturation, postsynaptic proteins like HOMER1 and ProSAP1/SHANK2 were unevenly distributed along the motoneuronal neurites on DIV 3. According to ongoing maturation, synaptic organization of HOMER1 and ProSAP1/SHANK2 increased and after 20 days in culture mature synapses were extensively found in primary motoneuron cultures (Fig. 16A,B)

Neuronal synapses, identified by the juxtaposed signals of pre- and postsynaptic proteins in immunoflourescence analysis were detected after 10 days in culture for the first time. 
A
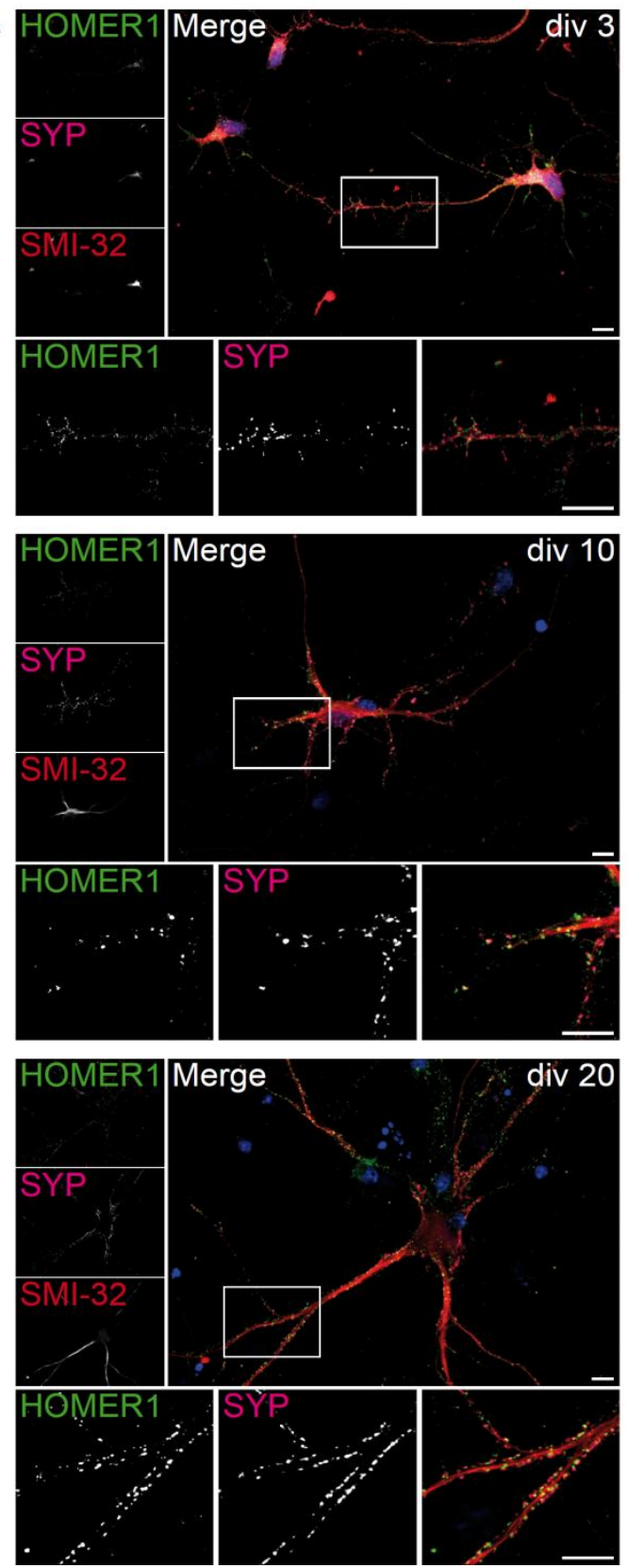

B
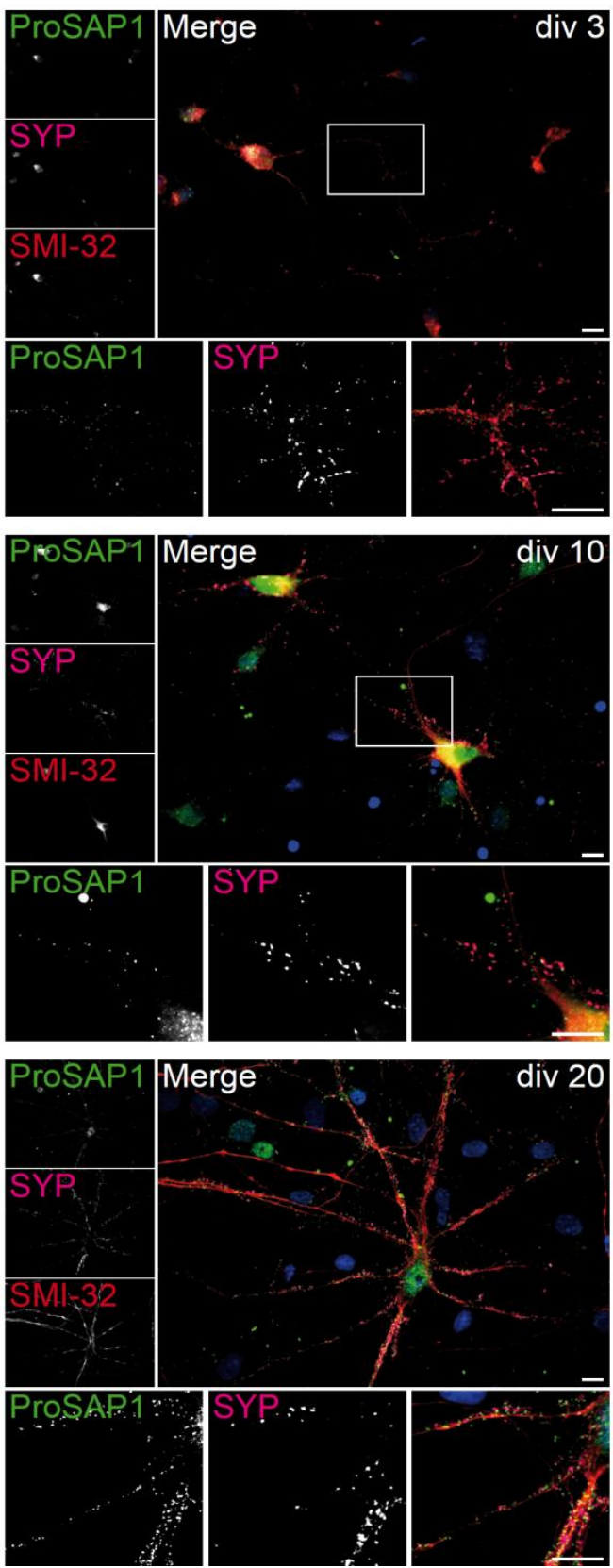

Figure 16 Developing Synapses in rat Motoneuron Cultures

(A,B) To investigate synaptogenesis, co-stainings against the presynaptic protein SYP (magenta) and the postsynaptic proteins HOMER1 and ProSAP1/SHANK2 (both green) were performed at three different time points of motoneuron (SMI32, red) development. At DIV 3 SYP was mainly localized in small-protrusions of the developing axon. The postsynaptic proteins were evenly distributed in the cytoplasm. Combined expression of SYP and HOMER1 / ProSAP1 within the developing synapses could be observed for the first time at DIV 10 indicating the formation of excitatory synapses. Colocalization of pre- and postsynaptic proteins steadily increased exemplarily shown up to 20 days in culture. Nuclei were stained with DAPI. All scale bars are $10 \mu \mathrm{m}$.

Quantitative studies of pre- and postsynaptic proteins further demonstrate the increasing amount of SYP as well as PCLO positive signals on DIV 20 . The number of excitatory synapses rapidly increased. On DIV 20, 6-7 SYP+ I HOMER1+ respectively SYP+ / ProSAP1+ signals per $30 \mu \mathrm{m}$ dendritic length could be detected in primary motoneuron cultures (Fig. 17C). 


\section{Results}

Transcript levels of SYP and PCLO were regulated in a very similar way. Both proteins reached their highest levels on DIV 3 and further decreased up to DIV 14

(Fig. 17A). mRNA levels of postsynaptic markers (HOMER 1 and ProSAP1/SHANK2) also peaked on DIV 3. Transcript levels for ProSAP1/SHANK2 presented a relatively high mRNA level already on DIV 1 in comparison to HOMER1, followed by a sharper mRNA reduction compared to HOMER1 (Fig. 17B)

A
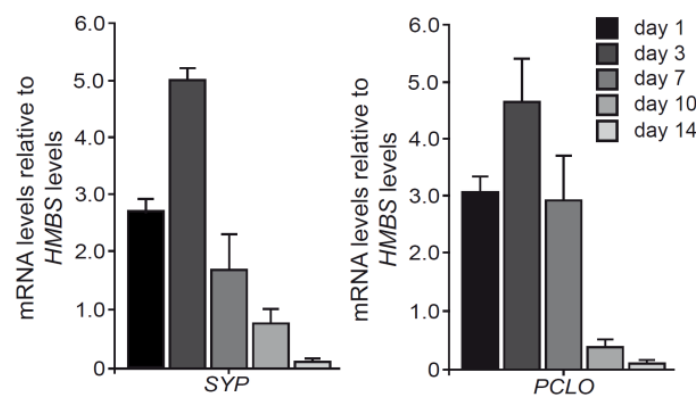

C

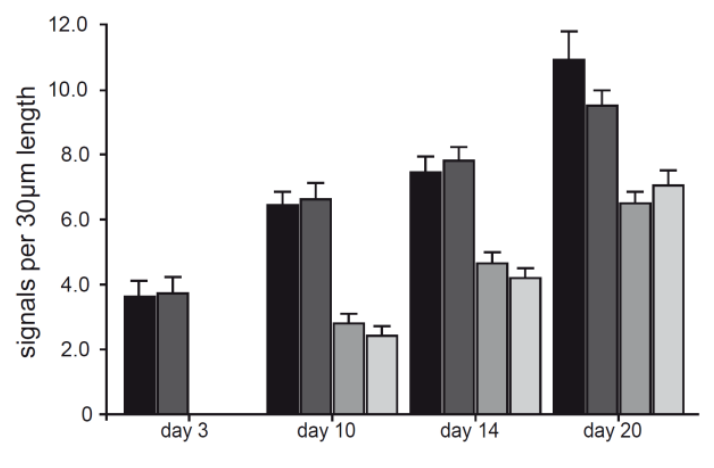

B
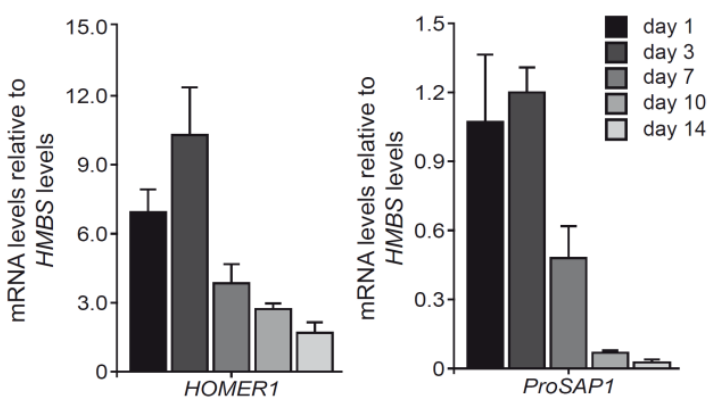

SYP $\square$ SYP/HOMER1
PCLO
SYP/PROSAP1

Figure 17 Quantification of Synapses in rat Motoneuron Cultures

(A) Regulation of mRNA-levels of presynaptic proteins: synaptophysin (SYP) and piccolo (PCLO). (B) Regulation of transcript levels of typical postsynaptic proteins: HOMER1 and ProSAP1/SHANK2. All investigated genes showed their highest mRNA level after 3 days in culture, followed by a continual decrease up to DIV 14. Expression levels are shown relative to the housekeeping gene HMBS ( $\mathrm{n}=4$ cells per time point). Error bars s.e.m. (C) For quantitative analysis of synapse number, SYP / PCLO positive signals and colocalization of SYP / HOMER1 and SYP / ProSAP1 were counted along a defined dendritic length $(30 \mu \mathrm{m}$, mean \pm s.e.m.; $n=20)$. 


\section{Discussion}

\section{Discussion}

Under chemically defined conditions hESCs and hiPSCs have been successfully differentiated to functional spinal motoneurons [82].

In the present study we focus on crucial hallmarks of maturation and synaptic formation of human-keratinocyte iPSC derived motoneurons. Such studies represent a basis for appropriate interpretation of distinct steps of neuronal development. The in vitro analysis of individualized human cells is considered to be a qualified tool with respect to new approaches in investigating pathological processes that lead to human motoneuron degeneration. Thus, an exact analysis of motoneuron development will be essential for future studies of patient specific cells in various pathogenetic settings.

\subsection{Generation of human pluripotent Stem Cells circumventing most ethical Concerns}

Human embryonic stem cells, which are derived from the ICM of the blastocyt provide the ability of self-renewal and are able to differentiate into somatic cell types of all three germ layers [195]. HESCs are also useful to generate models for human diseases and provide promising perspectives for screening new drugs. Homologous recombination recently has been described as a method to induce defined mutations in hESCs [232]. Homologous recombination of hESCs will be important for investigating pathogenesis of human diseases for which mouse models have proven inadequate. Moreover, it opens the prospect of repairing a gene defect by homologous recombination [232] or purifying specific hESCs derived gene types by controlled modifications of specific genes [232].

Though, hESCs may play a crucial role in future treatment of various diseases such as Parkinson's disease or diabetes [120, 196] . However, there are serious ethical difficulties concerning the use of human embryos as the derivation of pluripotent cells from oocyctes and embryos is fraught with disputes about the onset of human personhood [115].

Additionally, transplantation of non-autologous donor cells derived from hESCs would lead to immunological rejections. Immunological reactions arise when the transplanted cells are rejected by the recipient's immune system which destroys 
the transplanted tissue. This would have to be treated with the same drugs as it is usual in organ transplantation.

Some of these drawbacks were solved, when Yamanaka and Takahashi established the generation of induced pluripotent stem cells in 2006 . Somatic cells were reprogrammed by a lentiviral transduction of specific transcription factors including OCT4, SOX2 and KLF4 to induced pluripotent stem cells [192]. iPS cell morphology, proliferation, surface antigens and the epigenetic status of pluripotency associated genes are similar to those of embryonic stem cells [111]. Furthermore, induced pluripotent stem cells offer the chance to gain pluripotent cells from patients with various diseases and to study the cell biological backgrounds of pathogenesis [43, 156]. HiPSCs further represent an excellent tool to study the molecular mechanisms of pluripotency, early embryogenesis and cell development.

A variety of somatic cells with differing reprogramming capacity have been utilized for the generation of iPS cells [192, 221]. In 2008, Aasen and co-workers described a superior and non-invasive way of hiPSCs generation and could show that reprogramming of human keratinocytes from plucked hair is about 100-fold more efficient and 2 times faster compared with the reprogramming of human fibroblasts [1]. On the basis of these findings, we established the generation of induced pluripotent stem cells from human keratinocytes. HiPSCs derived from plucked human hair displayed all hallmarks of pluripotency and were able to differentiate in all three germ layers in vitro and in vivo [108]. However, the starting cell amount of human keratinocytes is limited and further optimization of the reprogramming protocol was necessary. In an additional study (Linta et al, [108]) we investigated the utilization of rat- instead of mouse embryonic fibroblasts as a feeder layer for the reprogramming process and obtained a significant, 3-times increased efficiency of the reprogramming process. Consequently, we established an easy to use and highly efficient reprogramming system which could be also very useful for the broad generation of hiPSCs.

\subsection{Stem cell model for human Motoneuron Maturation}

HESCs and hiPSCs provide the potential to differentiate in all cell and tissue types of the human body. Since the first description of the reprogramming of human 
somatic cells into hiPSCs a variety of more or less organotypic cell systems have been established. These systems might help in understanding cell type specific behavior e.g. development, proliferation, differentiation or even degeneration [40, 185, 198]. Cells differentiated from disease specific hiPSCs eventually feature a predicted phenotype. Differentiation of human iPSCs into motoneurons thus offers a new model for analyzing pathological processes which lead to motoneuron degeneration.

\subsubsection{Generation of hiPSCs derived Motoneurons}

The neural tube, which is formed during mammalian neurogenesis, consists of neural precursor cells expressing markers like PAX6 and SOX1. According to that knowledge, various studies reported that the generation of a homogenous population of SOX1+ / PAX6+ neuronal precursor (NP) cells is the first substantial step in neural differentiation of hiPSCs and hESCs [82, 230]. In our hands, NP cells are obtained by EB generation and replating of EBs under neurothrophic conditions. To obtain a pure population of NSCs and to get rid of cells differentiated into other lineages, neuronal rosettes are mechanically removed and cultured in the presence of neural growth factors.

During chick embryo development, a specific SHH and RA gradient leads to the development of OLIG2 positive motoneuron progenitors (pMN) [87]. We also found that the addition of $\mathrm{RA}$ and $\mathrm{SHH}$ in vitro promotes motoneuron differentiation and results in an accelerated transcription of motoneuron associated genes. These pMNs can be proliferated and kept in culture for up to 3 months. Recently, Brüstle and co-workers reported the derivation of a long-term, self-renewing neuroepithelial stem cell population from hESCs (It-hESNSCs) [97]. These IthESNSCs ensure a stable source of NSCs for neural differentiation with reduced batch-to-batch variations. Obtaining self-renewing pMNs would to an essential point in improving motoneuron differentiation protocols in the future.

Differentiation of pMNs into postmitotic motoneurons is mirrored by a downregulation of OLIG2 and an increased expression of HB9. In vitro, terminal differentiation is initiated by replating pMN-spheres after 28 days of differentiation. We can show that motoneurons generated from keratinocyte-derived hiPSCs carry all motoneuron specific markers including HB9, ILS1 and SMI-32. Additionally, the in vitro generated motoneurons feature all electrophysiological aspects of mature 


\section{Discussion}

neurons including the generation of action potentials and synaptic currents. A large proportion of motoneurons (40\%) can be detected two weeks after terminal plating of pMNs. This high purity of neural differentiation might also be related to the claimed somatic memory of hiPSCs [3, 152]. Thus, we successfully established motoneuron differentiation from keratinocyte-derived hiPSCs. In order to receive motoneuron cultures with a higher purity, hiPSCs lines could be generated in which a fluorophore is expressed under the control of e.g. the HB9 promoter. Differentiated motoneurons could easily be sorted to obtain $100 \%$ purity and fluorescent labeling of postmitotic motoneurons simplifies e.g. electrophysiological measurements (visibility of neurites).

\subsubsection{Maturation of hiPSCs derived Motoneurons}

A major problem in investigating the neuronal system is the lack of a defined in vitro model system and the deficiency of a common agreement upon "normal or physiological" developmental stages of human neurons. The variety of different cell types in the human CNS and the inaccessibility to primary human cells hamper an accurate characterization of defined neuronal cell types. This includes the exact time frame of distinct steps of motoneuron maturation including motoneuron specific gene expression, stage specific cellular morphology and formation of cell compartments such as the inter-neuronal or neuromuscular synapse. Physiological cell behavior and maturation has to be investigated before patient specific motoneuron with an eventually affected cellular development / morphology can be characterized. A pathogenic effect can only be judged based on an exact knowledge of non-pathogenic motoneuron behavior and its characteristics during development or functional state.

In our study, we investigate distinct steps of motoneuron maturation and the formation of neuronal and neuromuscular synapses. Although the efficiency of motoneuron derivation from hiPSCs is variable, the overall development and differentiation stages did not significantly differ among the used cell lines in our studies. This is crucial, as it was described recently that donor identity and sex influence differentiation efficiency [16]. Furthermore, it has been reported the motoneuron differentiation capacity of different iPSCs lines varies. Nevertheless, the time frame of individual development remains stable [83]. 
In a first step we focussed on the morphological maturation of hiPSCs derived motoneurons. We show that in vitro generated motoneurons gradually mature over time.

Shortly after plating, motoneurons become positive for SMI-32, a motoneuron specific neurofilament which is expressed already at very early stages. SMI-32 positive motoneurons also express HB9 after additional 3-5 days of differentiation and become positive for VAChT, required for the storage of ACh in synaptic vesicles.

We demonstrate the distinct and stepwise morphological change of motoneuron appearance. Shortly after plating, motoneurons present a bipolar morphology with growth cone like structures. According to ongoing differentiation a clear polarization into signal accepting (neurites) and signal transducing (axon) structures develop and neurites steadily elongated, branched and increased in number. Not only does the formation of neurites follow a specific time frame, but also the specification of the axon can be seen as a hallmark of neuronal development. After 3 weeks in culture, the axonal hillock is clearly defined followed by an axon devoid of Map2. These findings are underlined by the differentially expressed stem cell and neuron specific transcript levels over time.

As a second step, synaptogenesis was analyzed by investigation of time depended expression patterns and localization of synaptic proteins. During maturation, the formation of synapses, both interneuronal and neuromuscular, is initiated and matured connections are formed over time. According to ongoing maturation, expression of pre- and postsynaptic proteins alters from a diffuse pattern to a specific synaptic localization. First mature synaptic connections are detectable after 6 weeks in culture. In line with these findings, mRNA expression patterns are expressed in comparable time frames and their levels are regulated in a concerted manner. On ultrastructural level we observed a clear maturation of synaptic contacts. Importantly, synaptic contacts feature all crucial morphological aspects of excitatory synapses including an obvious PSD and an active zone featuring a large number of synaptic vesicles.

Along with the cellular and molecular changes, motoneurons become electrophysically active after 5 weeks in culture and synaptic currents are most readily detected with additional weeks in culture. This is likely due to enhanced synaptogenesis by the presence of spontaneously differentiating astrocytes [114]. 
We find that spontaneously differentiation into glial cells did not only promote synaptic formation, but also formed a myelin sheath around motoneuron neurites after 6-7 weeks of culture.

\subsubsection{Co-culture of hiPSCs derived Motoneurons and Myotubes}

Considering the great diversity of neurodegenerative conditions, there is growing evidence that both inter-neuronal and neuromuscular synapses as well as the distal axonal compartment are important subcellular pathological targets of MNDs $[37,57,59]$. With the help of animal models (e.g. SOD1 ${ }^{\text {G93A }}$ mouse) researchers have been able to examine the contribution of NMj breakdown to early stages of ALS pathogenesis [139]. In these mice, $40 \%$ of motor endplates lacked presynaptic motoneuron axon terminals at 47 days old, whereas mice of the same age showed no loss of lower motoneuron cell bodies in the ventral horn of the spinal cord. These mice did not become clinically weak until 80 days old [139].

Moreover, significant denervation of NMjs combined with a substantial accumulation of neurofilament proteins in the distal axons was reported in mouse models of spinal muscular atrophy (SMA) [59, 139].

Based on these findings, investigation of the formation of functional NMjs is a further aspect of the current study. To note, expression of VAChT and detection of spontaneous synaptic currents in electrophysiological measurements suggest functionality of in vitro differentiated motoneurons. Indeed, when co-cultured with primary rodent or human myotubes, motoneurons induce accumulation of the AChR (visualized by a-bungarotoxin staining) on the membrane surface of the myotubes. In single myotube cultures no AChR accumulation can be observed. Additionally, co-cultured primary myotubes show increased and more synchronous contractions compared to pure myotube cultures. In co-culture systems with human myotubes derived from muscle biopsies, no contractions could be observed which might be explained by an extended maturation of human myotubes compared to rodent cells. On ultrastructural level, typical features of NMjs such as the infolding of the postsynaptic membrane and vesicles-filled axon terminals can be detected.

We further investigated functionality of formed NMjs by inhibition of both the presynaptic transmitter release via botulinumtoxin A and the postsynaptic AChRs by curare (pancuronium) application. In both cases, myotube contractions are 


\section{Discussion}

significantly reduced indicating the formation of functional NMjs between hiPSCs derived motoneurons and primary myotubes.

Co-culture systems of hiPSCs derived motoneurons and myotubes provide the possibility to investigate the formation of the $\mathrm{NMj}$ of hiPSCs derived patient specific motoneurons and myotubes with the respect to a regular shape, protein expression and functionality.

Recently, differentiation of functional myotubes from mouse iPSCs by inducible overexpression of PAX7 (paired box gene 7) has been reported [36]. Following this findings, the next step in our studies will be the generation of a stably transduced, inducible PAX7-hiPSC line. Inducing PAX7 overexpression will hopefully promote differentiation of hiPSCs into myoblasts which can be purified via cell sorting and further differentiated into myotubes. This experimental set up provides the possibility to investigate NMjs between motoneurons and myotubes derived from the same donor or patient in different directions.

\subsubsection{Maturation of Motoneurons: Overview and Comparison}

Defined hallmarks of neurogenesis and synaptic formation of hiPSCs derived motoneurons are compared with the maturation of motoneurons derived from miPSCs and hESCs and to primary rodent motoneurons (Fig. 18).

We find that the development of human-keratinocytes iPSC derived motoneurons is quite similar to that of human embryonic stem cells in other studies. When the data from human studies are compared with the data from mouse ES or iPS cells, it becomes obvious that human neurons differentiate about ten times slower than neurons derived from mouse stem cells. These observations are further underlined by comparison with our data derived from rodent primary motoneuron cultures. 


\section{Discussion}

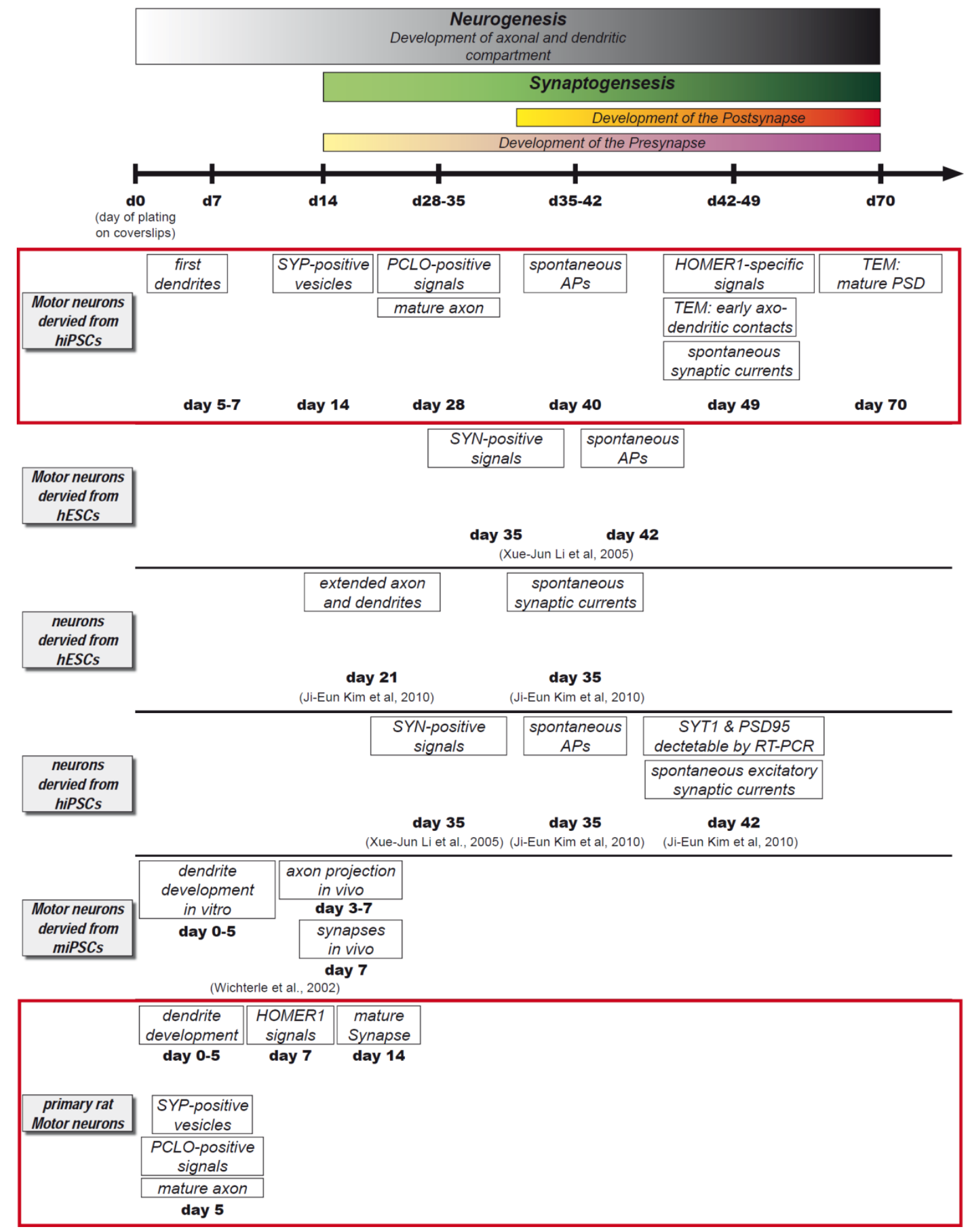

Figure 18 Time Frame of neuronal and synaptic maturation of hiPSCs derived Motoneurons

Comparisons of our findings with published data concerning neurogenesis and synaptic maturation. Compared groups consist of human keratinocytes iPS derived motoneurons (hiPSCs) evaluated in this study, MNs and unspecified neurons derived from human embryonic stem cells ( $\mathrm{hESCs}$ ), MNs derived from mouse iPSCs and primary rat spinal MNs embryonic day E14.5. Subject of the comparisons are (beginning at day 0 until day 70 after plating) neuronal maturation, synaptogenesis and distinct time dependent neuronal / synaptic features over time, such as pre- and postsynaptic development and abundance of electrophysiological hallmarks.

Abbreviations: $d$ = day; hiPSC = human induced pluripotent stem cells; SYP = Synaptophysin; PCLO = Piccolo; AP = Action Potential; TEM = transmission electron microscope; PSD = Postsynaptic Density; SYN = Synapsin; SYT1 = Synaptotagmin1; Cited studies were: [82] 


\section{Discussion}

\subsubsection{Improvement of Culture Conditions}

Differentiation of hiPSCs into functional motoneurons is achieved using a modified protocol adapted from [82].

From day 15 of the differentiation protocol (after lifting off the neuronal rosettes), pMNs grow quickly and can be easily expanded [193]. However, if pMNs have been in culture for up to 8 weeks without plating cells for terminal differentiation, we found that their proliferative capacity is reduced. To deal with this problem, we added leukemia inhibitory factor (LIF, 1:100, endconcentration $10^{5}$ units / ml) immediately after dissociation of the cells for 48 hours. LIF is known to promote proliferation of hNSCs synergistically to FGF and EGF [193]. We also observed an enhancement of pMNs proliferation after dissociation and an enhanced sphere size as early as 1 day after passaging. As it has also been reported that hNSCs cultured under different growth factors vary in their phenotypic differentiation pattern [82], we supplied LIF only after dissociation for $48 \mathrm{~h}$. To verify the accelerated proliferation of pMNs after LIF treatment proliferation assays should be performed in further studies. Before LIF is used routinely for long-term pMN culture, its influence on pMNs' differentiation into post-mitotic motoneurons should also be investigated.

In our studies, hiPSCs derived motoneurons are kept in culture for more than 20 weeks. To improve cell survival we tested different coatings and culture dishes. In the end, we retained to the PLO / laminin coating recommended in the protocol of Bao-Yang Hu and Su-Chun Zhang [88, 114]. But we observed, that for long-term culture (> 4 weeks) treated ibidis (35 $\mathrm{mm}$ high, tissue culture treated) approve the highest cell survival.

Glial cells also seem to play a crucial role in cell survival and acceleration of synaptic integration $[88,228]$. To improve in vitro generated motoneuron survival and promote synapse formation co-culture of human motoneurons and glial cells would be useful. It has been reported in hESC neural differentiation cultures that astrocytes appear after the differentiation of neurons [81, 229] reflecting early neurogenesis followed by gliagenesis at a later time. Successful differentiation of oligodendrocytes and astrocytes has already been reported [40]. Thus, co-culture of motoneurons and glial cells differentiated from hiPSCs from the same donor would be helpful. Moreover, it has been reported that glial cells carrying ALS causing mutations influence hESC derived human motoneurons [89]. Based on 
these findings co-culture of hiPSCs derived glial cells and motoneurons offer not only improved culture conditions but also new approaches to investigate motoneuron disease pathogenesis in the future.

\subsection{The Stem Cell Model and Motoneuron Degeneration}

Motoneurons are of particular interest for studies dealing with degenerative disorders which are summarized as motoneuron diseases (MNDs). These disorders of the motoneuron system include well known degenerative diseases such as ALS, SMA or developmental defects combined with degenerative symptoms as in Kennedy's disease.

MNDs are mainly age-related, largely limited to humans and rarely occur spontaneously in animals. Although aged nonhuman primates and some other higher-order animal species show aspects similar to those of human brain aging, these animals generally do not readily develop the full neuropathological or clinical phenotypes seen in humans [89]. Differences in chaperons, proteasomal and autophagosmal clearance mechanisms, lifespan or comorbid conditions are considered causes for the apparent resistance of nonhumans to age-related neurodegenerative diseases [22]. Thus, no efficient animal models exist for agerelated neurodegenerative diseases such as ALS.

Animal models represent essential model systems for our current understanding of human disease pathogenesis. As most MNDs do not naturally occur in commonly used laboratory animals, pathogenic aspects of human MNDs have to be artificially introduced into laboratory animals. Introduction of human mutant SOD1 leads to a phenotype similar to human ALS [22, 114]. In these cases, expression of multiple copies of mutant human SOD1 is necessary to provoke a phenotype $[60,114]$. Contrary to that, only one single copy of mutant SOD1 results in an ALS phenotype in human patients. The most common form of mutated SOD1 in human ALS patients, the alanine to valine substitution (A4V), does not lead to a phenotype in mice [114]. Therefore, differences exist between human and mice in supporting the pathogenic potential of human SOD1 [28].

A number of MND animal models have been investigated, including zebra fish (Danio Rerio), mice and fruit flies (Drosophila Melanogaster). Although they often display neuronal dysfunction typical for ALS - e.g. fruit flies exhibit lethargy [100, 
210] and zebrafish lack appropriate axonal development [31]. Nevertheless - none of these models exhibit the same complexity that exists in a mammalian system, e.g. with numerous cell-to-cell interactions between supporting cells, motoneurons and the muscle itself. Moreover, for many monogenic diseases (e.g. Lesch-Nyhan or Duchenne muscular dystrophy), the animal models show no or only an approximate resemblance to the human disease [32].

Drugs, which had been shown to be efficacious for the treatment of ALS in mouse models (e.g. minocycline), had no beneficial effects in human clinical trials. Minocycline even hastened ALS progression [114].

Although investigation of ALS associated animal models enlightened various aspects of ALS pathogenesis, the relevance of these findings to the human ALS pathogenesis is still discussed. Thus, model systems with a human background would complete the current knowledge about motoneuron degeneration.

New ALS model systems will be created on the basis of human stem cells. HESCs lines were transduced with a lentiviral vector with a built-in CRE-loxP cassette to screen for gene loci resistant to transgenic silencing. The generated hESCs line is relatively resistant to transgenic silencing and due to the built-in CRE-loxP cassette any gene of interest such as ALS causing genes can be introduced, via CRE-mediated recombination [192]. These genetically altered hESCs can now be differentiated to cells from all three germ layers including motoneurons and glial cells.

The establishment of iPSCs by Yamanaka and colleagues [41, 50] offers the possibility to generate patient specific human stem cells. HiPSCs have successfully been generated from ALS and SMA patients and differentiated into functional motoneurons [17]. Patient specific hiPSCs could provide a new model to study cellular and molecular mechanisms of motoneuron degeneration. Different targets of motoneuron degeneration which have been found in animal models can now be investigated in the human system.

Many genetic diseases show variable penetrance and different clinical symptoms from patient to patient. This lack of consistence is based on the complex influence of the genetic background and the environment. Patient specific hiPSCs combined with the knowledge of the clinical history of the donor provide personalized experimental set ups and interpretation. Differences among hiPSCs lines caused by different epigenetic background or environment can be reduced by comparing 
affected cells with familiar healthy controls. So, establishing appropriate and well characterized control hiPSC lines is an essential step before interpreting eventual pathogenic hallmarks of patient specific cells.

A particular challenge of the hiPSCs based model system arises when the disease is non-cell autonomous. Although hiPSCs can be differentiated into several cell types of the CNS, it is still impossible to mimic the mixture of different neurons, glia and microglia of the human brain or spinal cord. However, new co-culture systems - e.g. motoneurons and myotubes or glial cells differentiated from the same donor - provide new approaches and supply detailed investigation of affected cells together with their natural counterpart. Disease models of derived from hiPSCs that manifest a robust cellular phenotype can be used for drug screenings as well as testing drugs found to be effective in animal models.

Nevertheless, a pathogenic effect can only be judged with the exact knowledge of the normal motoneuron behavior and maturation. We analyzed motoneuron maturation and the functional interplay of neurons and myotubes to provide a study which might be helpful in the future to characterize development and behavior of affected, patient specific motoneurons.

\subsection{Read out Systems for human Motoneuron Degeneration}

In human MND patients motoneuron degeneration usually occurs in adult life suggesting that pathogenic phenotypes may not manifest in motoneurons or stem cells derived from patients suffering ALS, SMA or Kennedy's disease. Thus, read out systems have to be established to detect or to provoke a disease specific phenotype. Motoneurons carrying MND-causing mutations could be investigated with respect to morphological changes (alterations in the cytoskeleton, axonal length and synapse formation) and to possibly changes in electrophysiological features including changes in the resting potential, in the generation of action potentials, synaptic currents and an altered receptor composition.

\subsubsection{Long-term Culture and Stress Response of human Motoneurons}

Specific cellular cascades including mitochondria dysfunction and excitotoxicity contribute to programmed cell death in aging motoneurons have been considered to play crucial roles in ALS pathogenesis [59, 61]. Long-term culture of human 


\section{Discussion}

motoneurons derived from hiPSCs of ALS patients mimicking aging of motoneurons could be investigated with respect to alterations of cell survival and apoptosis.

Previous studies also described an impaired retrograde axonal transport in MND animal models resulting in an accumulation of axonal transported proteins in the distal ends of motoneuron axons [124, 173]. Consequently, axonal transport in long-term motoneuron cultures is another important target in MND research.

Impaired mitochondrial dysfunctions have often been reported to contribute to ALS pathogenesis [124]. In neurons, mitochondria form a highly dynamic and interconnected network that undergoes transport and remodeling by fusion and fission [123, 124]. It has already been shown that mitochondrial impairment leads to the failure to provide healthy mitochondria to synaptic terminals and neuromuscular junctions in mutant SOD1 mice [128]. Analysis of the mitochondrial function in patient specific hiPSCs derived motoneurons alone and/or co-cultured with myotubes is another interesting topic. Furthermore, there are several proposed mechanisms of toxicity, which include protein aggregation, neuroinflammation, growth factor deficiency or pathologies in surrounding cells like glial or muscle cells which can be investigated in long-term culture of hiPSCs derived motoneurons of MND patients and healthy controls. Additional interventions such as oxidative stress or excitotoxic stress may be necessary to provoke a pathological phenotype.

Alternatively, generation of stably transduced hiPSCs lines carrying multiple copies of disease genes or in vitro induction of pathologic genes may accelerate the pathogenic processes. Using vector systems which allow regulation of the transcription of the target genes by e.g. doxycyclin application at specific time points of motoneuron development could not only be used to force pathological processes but also as control or rescue systems in comparison to patient specific cells.

\subsubsection{Co-culture Systems}

An additional advantage of culture systems is that they allow single or co-culture of different cells types, which helps analyzing cell specific contributions and cellular interactions to pathological processes. 
Recent studies have shown that co-culturing hESC-derived motoneurons with human primary astrocytes expressing SOD1 [40] or mouse primary glial cells carrying SOD1 mutations [81, 229] results in motoneuron death but does not affect other neuronal subtypes. Consequently, co-culturing hiPSCs derived motoneurons together with hiPSCs derived oligodendrocytes [58, 139, 198] from the same donor, provides the ability to investigate not only basic interactions between glial cell and motoneurons in a human system but also the influence of mutated respectively unaffected glial cells on patient derived motoneurons.

Recent evidence from systematic studies and human patients indicate that the NMjs is an important early target in MNDs, featuring functional and morphological abnormalities previous to pathological changes of the motoneurons [47]. The molecular composition of the NMj is crucial for its function and maintenance whereas dysregulation of endplate physiology is considered to be involved in motoneuron degeneration. The establishment of reprogramming technologies has opened up the chance to generate human NMjs in vitro using hiPSCs from healthy individuals and from MND patients. Human muscle tissue could easily be derived from muscular biopsies or even differentiated from hiPSCs. The influence of affected or healthy muscle cells on motoneurons derived from MND patients could be studied in disease-specific human motoneuron-muscle co-culture systems.

In our studies we have already demonstrated that motoneurons derived from hiPSCs are able to from NMjs which are functional active and feature all crucial morphological aspects of neuromuscular connections.

\subsection{Conclusion}

To date, most of the studies on the human brain and neuronal function have been assessed on postmortem tissues that were often not well preserved and only demonstrated the end-stage of neurodegenerative diseases. Furthermore, animal models available to study human neurological diseases are limited in recapitulating human neuropathological phenotypes. However, a detailed investigation of distinct steps of normal motoneuron maturation including stage dependent gene expression, differentiation of the dendritic and axonal compartment and formation of neuronal and neuromuscular synapses is inevitable for future analysis of patient specific cells. In our study we demonstrate that 


\section{Discussion}

human keratinocytes can efficiently be reprogrammed into induced pluripotent stem cells and subsequently be differentiated into functional motoneurons.

We define hallmarks of motoneuron development and formation of neuronal and neuromuscular synapses. Furthermore we describe stage dependent gene expression not only of motoneuron specific genes but also for various pre- and postsynaptic genes. On ultrastructural level we show that emerged synapses feature all crucial morphological aspects not only of excitatory synapses but also of neuromuscular junctions. Additionally, functionality of motoneurons is convincingly shown via electrophysiological measurements and by further inhibition of inter-neuronal and neuromuscular synaptic transmission. These findings now provide an important tool to study the cell biology and molecular aspects of embryonic motoneuron development and MND pathogenesis. 


\section{Summary}

\section{Summary}

Motoneuron diseases are a subfamily of the large entity of neurodegenerative disorders. ALS (amyotrophic lateral sclerosis) is the most common adult onset disorder of motoneurons and is characterized by atrophy and degeneration of the upper and the lower motoneuron. In the last decades, animal models and in vitro cell models of rodent motoneurons have presented the main model systems in MND (motoneuron disease) research. However, animal models are still restricted in reflecting human MND pathogenesis. Therefore, stem cell based model systems have been established to investigate cellular and molecular aspects of pathological processes.

In our study, we demonstrate that human keratinocytes can efficiently be reprogrammed to hiPSCs (human induced pluripotent stem cells) featuring all crucial aspects of pluripotency. Furthermore, we provide a slightly improved differentiation protocol leading to human motoneuron cultures with a minimum of $40 \%$ purity. In order to provide a study which could be helpful for appropriate interpretation of patient specific cell behavior, we define hallmarks of neurogenesis and synaptogenesis of hiPSCs derived motoneurons.

We primarily focus on motoneuron maturation and show specific aspects of axonal and dendritic development, further underlined by mRNA (messenger ribonucleic acid) expression patterns. In a next step, we analyze progression of synaptic formation and provide important insights into the stage depended expression and localization of pre- and postsynaptic proteins. After 7 weeks in culture hiPSCs derived motoneurons feature all major electrophysiological properties of mature neurons including formation of action potentials and generation of synaptic currents. Further analyses reveal that synaptic currents are reversibly suppressed by an AMPA receptor antagonist, indicating the existence of functionally operating glutamatergic synapses. Moreover, hiPSCs derived motoneurons co-cultured with skeletal muscle cells initiate the formation of $\mathrm{NMjs}$ (neuromuscular junctions) displaying all functional and morphological principles of mature synaptic connections between motoneurons and myotubes.

We could show that the development of hiPSCs derived motoneurons is comparable to motoneuron derived from hESCs (human embryonic stem cells). Moreover, we study the maturation of rodent motoneurons with respect to the 
same hallmarks of maturation as we focused on in the human system. Comparing the data from both studies we show that the development of human motoneurons is about 10 times slower than the development of rodent motoneurons. This is further verified by comparing the data from human studies with data from mouse ES or iPS cells.

We provide a study which might be a basis for appropriate interpretation and further characterization of motoneuron development and the assessment of pathogenic motoneuron behavior in vitro. 


\section{List of Literature}

1. Aasen T, Raya A, Barrero MJ, Garreta E, Consiglio A, Gonzalez F, Vassena R, Bilic J, Pekarik V, Tiscornia G, Edel M, Boue S, Izpisua Belmonte JC (2008) Efficient and rapid generation of induced pluripotent stem cells from human keratinocytes. Nat Biotechnol 26:1276-1284

2. Amit M, Carpenter MK, Inokuma MS, Chiu CP, Harris CP, Waknitz MA, Itskovitz-Eldor J, Thomson JA (2000) Clonally derived human embryonic stem cell lines maintain pluripotency and proliferative potential for prolonged periods of culture. Dev Biol 227:271-278

3. Bar-Nur O, Russ HA, Efrat S, Benvenisty N (2011) Epigenetic memory and preferential lineage-specific differentiation in induced pluripotent stem cells derived from human pancreatic islet Beta cells. Cell Stem Cell 9:17-23

4. Baron MK, Boeckers TM, Vaida B, Faham S, Gingery M, Sawaya MR, Salyer D, Gundelfinger ED, Bowie JU (2006) An architectural framework that may lie at the core of the postsynaptic density. Science 311:531-535

5. Battistini S, Giannini F, Greco G, Bibbo G, Ferrera L, Marini V, Causarano R, Casula M, Lando G, Patrosso MC, Caponnetto C, Origone P, Marocchi A, Del Corona A, Siciliano G, Carrera P, Mascia V, Giagheddu M, Carcassi C, Orru S, Garre C, Penco S (2005) SOD1 mutations in amyotrophic lateral sclerosis. Results from a multicenter Italian study. J Neurol 252:782-788

6. Begemann G, Marx M, Mebus K, Meyer A, Bastmeyer M (2004) Beyond the neckless phenotype: influence of reduced retinoic acid signaling on motor neuron development in the zebrafish hindbrain. Dev Biol 271:119-129

7. Beyhan Z, lager AE, Cibelli JB (2007) Interspecies nuclear transfer: implications for embryonic stem cell biology. Cell Stem Cell 1:502-512

8. Bischoff R, Heintz C (1994) Enhancement of skeletal muscle regeneration. Dev Dyn 201:41-54

9. Blasi J, Chapman ER, Link E, Binz T, Yamasaki S, De Camilli P, Sudhof TC, Niemann H, Jahn R (1993) Botulinum neurotoxin A selectively cleaves the synaptic protein SNAP-25. Nature 365:160-163

10. Blau HM, Blakely BT (1999) Plasticity of cell fate: insights from heterokaryons. Semin Cell Dev Biol 10:267-272

11. Boeckers TM, Kreutz MR, Winter C, Zuschratter W, Smalla KH, SanmartiVila L, Wex H, Langnaese K, Bockmann J, Garner CC, Gundelfinger ED (1999) Proline-rich synapse-associated protein-1/cortactin binding protein 1 (ProSAP1/CortBP1) is a PDZ-domain protein highly enriched in the postsynaptic density. J Neurosci 19:6506-6518

12. Boeckers TM, Bockmann J, Kreutz MR, Gundelfinger ED (2002) ProSAP/Shank proteins - a family of higher order organizing molecules of the postsynaptic density with an emerging role in human neurological disease. J Neurochem 81:903-910

13. Boeckers TM (2006) The postsynaptic density. Cell Tissue Res 326:409422

14. Boillee S, Vande Velde C, Cleveland DW (2006) ALS: a disease of motor neurons and their nonneuronal neighbors. Neuron 52:39-59

15. Borowiak M, Maehr R, Chen S, Chen AE, Tang W, Fox JL, Schreiber SL, Melton DA (2009) Small molecules efficiently direct endodermal differentiation of mouse and human embryonic stem cells. Cell Stem Cell 4:348-358 
16. Boulting GL, Kiskinis E, Croft GF, Amoroso MW, Oakley DH, Wainger BJ, Williams DJ, Kahler DJ, Yamaki M, Davidow L, Rodolfa CT, Dimos JT, Mikkilineni S, MacDermott AB, Woolf CJ, Henderson CE, Wichterle H, Eggan K (2011) A functionally characterized test set of human induced pluripotent stem cells. Nat Biotechnol 29:279-286

17. Boutahar N, Wierinckx A, Camdessanche JP, Antoine JC, Reynaud E, Lassabliere F, Lachuer J, Borg J (2011) Differential effect of oxidative or excitotoxic stress on the transcriptional profile of amyotrophic lateral sclerosis-linked mutant SOD1 cultured neurons. J Neurosci Res 89:14391450

18. Brambrink T, Hochedlinger K, Bell G, Jaenisch R (2006) ES cells derived from cloned and fertilized blastocysts are transcriptionally and functionally indistinguishable. Proc Natl Acad Sci U S A 103:933-938

19. Brennand KJ, Simone A, Jou J, Gelboin-Burkhart C, Tran N, Sangar S, Li Y, Mu Y, Chen G, Yu D, McCarthy S, Sebat J, Gage FH (2011) Modelling schizophrenia using human induced pluripotent stem cells. Nature 473:221225

20. Brookhart JM (1952) A study of corticospinal activation of motor neurons. Res Publ Assoc Res Nerv Ment Dis 30:157-173

21. Brooks BR (1996) Natural history of ALS: symptoms, strength, pulmonary function, and disability. Neurology 47:S71-81; discussion S81-72

22. Bruijn LI, Miller TM, Cleveland DW (2004) Unraveling the mechanisms involved in motor neuron degeneration in ALS. Annu Rev Neurosci 27:723749

23. Buehr M, Meek S, Blair K, Yang J, Ure J, Silva J, McLay R, Hall J, Ying QL, Smith A (2008) Capture of authentic embryonic stem cells from rat blastocysts. Cell 135:1287-1298

24. Campbell KH, Fisher P, Chen WC, Choi I, Kelly RD, Lee JH, Xhu J (2007) Somatic cell nuclear transfer: Past, present and future perspectives. Theriogenology 68 Suppl 1:S214-231

25. Carpenter MK, Inokuma MS, Denham J, Mujtaba T, Chiu CP, Rao MS (2001) Enrichment of neurons and neural precursors from human embryonic stem cells. Exp Neurol 172:383-397

26. Chen G, Gulbranson DR, Hou Z, Bolin JM, Ruotti V, Probasco MD, SmugaOtto K, Howden SE, Diol NR, Propson NE, Wagner R, Lee GO, Antosiewicz-Bourget J, Teng JM, Thomson JA (2011) Chemically defined conditions for human iPSC derivation and culture. Nat Methods 8:424-429

27. Chen S, Borowiak M, Fox JL, Maehr R, Osafune K, Davidow L, Lam K, Peng LF, Schreiber SL, Rubin LL, Melton D (2009) A small molecule that directs differentiation of human ESCs into the pancreatic lineage. Nat Chem Biol 5:258-265

28. Chen Y, Yang M, Deng J, Chen X, Ye Y, Zhu L, Liu J, Ye H, Shen Y, Li Y, Rao EJ, Fushimi K, Zhou X, Bigio EH, Mesulam M, Xu Q, Wu JY (2011) Expression of human FUS protein in Drosophila leads to progressive neurodegeneration. Protein Cell 2:477-486

29. Childs AJ, Kinnell HL, Collins CS, Hogg K, Bayne RA, Green SJ, McNeilly AS, Anderson RA (2010) BMP signaling in the human fetal ovary is developmentally regulated and promotes primordial germ cell apoptosis. Stem Cells 28:1368-1378

30. Churchill FB (1991) The rise of classical descriptive embryology. Dev Biol (N Y 1985) 7:1-29 
31. Colman A, Dreesen O (2009) Pluripotent stem cells and disease modeling. Cell Stem Cell 5:244-247

32. Couzin J (2007) Clinical research. ALS trial raises questions about promising drug. Science 318:1227

33. Cowan CA, Atienza J, Melton DA, Eggan K (2005) Nuclear reprogramming of somatic cells after fusion with human embryonic stem cells. Science 309:1369-1373

34. Craig AM, Banker G (1994) Neuronal polarity. Annu Rev Neurosci 17:267310

35. Dale HH, Feldberg W, Vogt M (1936) Release of acetylcholine at voluntary motor nerve endings. J Physiol 86:353-380

36. Darabi R, Pan W, Bosnakovski D, Baik J, Kyba M, Perlingeiro RC (2011) Functional myogenic engraftment from mouse iPS cells. Stem Cell Rev 7:948-957

37. David G, Nguyen K, Barrett EF (2007) Early vulnerability to ischemia/reperfusion injury in motor terminals innervating fast muscles of SOD1-G93A mice. Exp Neurol 204:411-420

38. de Castro BM, De Jaeger X, Martins-Silva C, Lima RD, Amaral E, Menezes C, Lima P, Neves CM, Pires RG, Gould TW, Welch I, Kushmerick C, Guatimosim C, Izquierdo I, Cammarota M, Rylett RJ, Gomez MV, Caron MG, Oppenheim RW, Prado MA, Prado VF (2009) The vesicular acetylcholine transporter is required for neuromuscular development and function. Mol Cell Biol 29:5238-5250

39. Dhara SK, Stice SL (2008) Neural differentiation of human embryonic stem cells. J Cell Biochem 105:633-640

40. Di Giorgio FP, Boulting GL, Bobrowicz S, Eggan KC (2008) Human embryonic stem cell-derived motor neurons are sensitive to the toxic effect of glial cells carrying an ALS-causing mutation. Cell Stem Cell 3:637-648

41. Dimos JT, Rodolfa KT, Niakan KK, Weisenthal LM, Mitsumoto $H$, Chung W, Croft GF, Saphier G, Leibel R, Goland R, Wichterle H, Henderson CE, Eggan K (2008) Induced pluripotent stem cells generated from patients with ALS can be differentiated into motor neurons. Science 321:1218-1221

42. Ding VM, Ling L, Natarajan S, Yap MG, Cool SM, Choo AB (2010) FGF-2 modulates Wnt signaling in undifferentiated hESC and iPS cells through activated PI3-K/GSK3beta signaling. J Cell Physiol 225:417-428

43. Do JT, Joo JY, Han DW, Arauzo-Bravo MJ, Kim MJ, Greber B, Zaehres H, Sobek-Klocke I, Chung HM, Scholer HR (2009) Generation of parthenogenetic induced pluripotent stem cells from parthenogenetic neural stem cells. Stem Cells 27:2962-2968

44. Dresbach T, Hempelmann A, Spilker C, tom Dieck S, Altrock WD, Zuschratter W, Garner CC, Gundelfinger ED (2003) Functional regions of the presynaptic cytomatrix protein bassoon: significance for synaptic targeting and cytomatrix anchoring. Mol Cell Neurosci 23:279-291

45. Du ZW, Zhang SC (2004) Neural differentiation from embryonic stem cells: which way? Stem Cells Dev 13:372-381

46. Dupuis L, Gonzalez de Aguilar JL, Echaniz-Laguna A, Eschbach J, Rene F, Oudart H, Halter B, Huze C, Schaeffer L, Bouillaud F, Loeffler JP (2009) Muscle mitochondrial uncoupling dismantles neuromuscular junction and triggers distal degeneration of motor neurons. PLoS One 4:e5390 
47. Dupuis L, Loeffler JP (2009) Neuromuscular junction destruction during amyotrophic lateral sclerosis: insights from transgenic models. Curr Opin Pharmacol 9:341-346

48. Dvash T, Mayshar Y, Darr H, McElhaney M, Barker D, Yanuka O, Kotkow KJ, Rubin LL, Benvenisty N, Eiges R (2004) Temporal gene expression during differentiation of human embryonic stem cells and embryoid bodies. Hum Reprod 19:2875-2883

49. Dvorak P, Dvorakova D, Koskova S, Vodinska M, Najvirtova M, Krekac D, Hampl A (2005) Expression and potential role of fibroblast growth factor 2 and its receptors in human embryonic stem cells. Stem Cells 23:1200-1211

50. Ebert AD, Yu J, Rose FF, Jr., Mattis VB, Lorson CL, Thomson JA, Svendsen CN (2009) Induced pluripotent stem cells from a spinal muscular atrophy patient. Nature 457:277-280

51. Erceg S, Lainez S, Ronaghi M, Stojkovic P, Perez-Arago MA, MorenoManzano V, Moreno-Palanques R, Planells-Cases R, Stojkovic M (2008) Differentiation of human embryonic stem cells to regional specific neural precursors in chemically defined medium conditions. PLoS One 3:e2122

52. Ericson J, Thor S, Edlund T, Jessell TM, Yamada T (1992) Early stages of motor neuron differentiation revealed by expression of homeobox gene Islet-1. Science 256:1555-1560

53. Ericson J, Morton S, Kawakami A, Roelink H, Jessell TM (1996) Two critical periods of Sonic Hedgehog signaling required for the specification of motor neuron identity. Cell 87:661-673

54. Evans MJ, Kaufman MH (1981) Establishment in culture of pluripotential cells from mouse embryos. Nature 292:154-156

55. Feng B, Jiang J, Kraus P, Ng JH, Heng JC, Chan YS, Yaw LP, Zhang W, Loh YH, Han J, Vega VB, Cacheux-Rataboul V, Lim B, Lufkin T, Ng HH (2009) Reprogramming of fibroblasts into induced pluripotent stem cells with orphan nuclear receptor Esrrb. Nat Cell Biol 11:197-203

56. Fenster SD, Chung WJ, Zhai R, Cases-Langhoff C, Voss B, Garner AM, Kaempf U, Kindler S, Gundelfinger ED, Garner CC (2000) Piccolo, a presynaptic zinc finger protein structurally related to bassoon. Neuron 25:203-214

57. Fischer LR, Culver DG, Tennant $P$, Davis AA, Wang M, CastellanoSanchez A, Khan J, Polak MA, Glass JD (2004) Amyotrophic lateral sclerosis is a distal axonopathy: evidence in mice and man. Exp Neurol 185:232-240

58. Frey D, Schneider C, Xu L, Borg J, Spooren W, Caroni P (2000) Early and selective loss of neuromuscular synapse subtypes with low sprouting competence in motoneuron diseases. J Neurosci 20:2534-2542

59. Frugier T, Tiziano FD, Cifuentes-Diaz C, Miniou P, Roblot N, Dierich A, Le Meur M, Melki J (2000) Nuclear targeting defect of SMN lacking the Cterminus in a mouse model of spinal muscular atrophy. Hum Mol Genet 9:849-858

60. Furukawa Y, Fu R, Deng HX, Siddique T, O'Halloran TV (2006) Disulfide cross-linked protein represents a significant fraction of ALS-associated $\mathrm{Cu}$, $\mathrm{Zn}$-superoxide dismutase aggregates in spinal cords of model mice. Proc Natl Acad Sci U S A 103:7148-7153

61. Gallo JM (2001) Kennedy's disease: a triplet repeat disorder or a motor neuron disease? Brain Res Bull 56:209-214 
62. Garner CC, Waites CL, Ziv NE (2006) Synapse development: still looking for the forest, still lost in the trees. Cell Tissue Res 326:249-262

63. Gauthaman K, Fong CY, Bongso A (2010) Effect of ROCK inhibitor Y27632 on normal and variant human embryonic stem cells (hESCs) in vitro: its benefits in hESC expansion. Stem Cell Rev 6:86-95

64. Gavrilov S, Marolt D, Douglas NC, Prosser RW, Khalid I, Sauer MV, Landry DW, Vunjak-Novakovic G, Papaioannou VE (2011) Derivation of two new human embryonic stem cell lines from nonviable human embryos. Stem Cells Int 2011:765378

65. Genoux D, Montgomery JM (2007) Glutamate receptor plasticity at excitatory synapses in the brain. Clin Exp Pharmacol Physiol 34:1058-1063

66. Gerrow K, Romorini S, Nabi SM, Colicos MA, Sala C, El-Husseini A (2006) A preformed complex of postsynaptic proteins is involved in excitatory synapse development. Neuron 49:547-562

67. Grabrucker A, Vaida B, Bockmann J, Boeckers TM (2009) Synaptogenesis of hippocampal neurons in primary cell culture. Cell Tissue Res 338:333341

68. Grabrucker AM, Knight MJ, Proepper C, Bockmann J, Joubert M, Rowan M, Nienhaus GU, Garner CC, Bowie JU, Kreutz MR, Gundelfinger ED, Boeckers TM (2011) Concerted action of zinc and ProSAP/Shank in synaptogenesis and synapse maturation. Embo J 30:569-581

69. Gray EG (1959) Electron microscopy of synaptic contacts on dendrite spines of the cerebral cortex. Nature 183:1592-1593

70. Greenway MJ, Andersen PM, Russ C, Ennis S, Cashman S, Donaghy C, Patterson V, Swingler R, Kieran D, Prehn J, Morrison KE, Green A, Acharya KR, Brown RH, Jr., Hardiman O (2006) ANG mutations segregate with familial and 'sporadic' amyotrophic lateral sclerosis. Nat Genet 38:411413

71. Gundelfinger ED, tom Dieck S (2000) Molecular organization of excitatory chemical synapses in the mammalian brain. Naturwissenschaften 87:513523

72. Gurney ME, Pu H, Chiu AY, Dal Canto MC, Polchow CY, Alexander DD, Caliendo J, Hentati A, Kwon YW, Deng HX, et al. (1994) Motor neuron degeneration in mice that express a human $\mathrm{Cu}, \mathrm{Zn}$ superoxide dismutase mutation. Science 264:1772-1775

73. Habisch HJ, Liebau S, Lenk T, Ludolph AC, Brenner R, Storch A (2010) Neuroectodermally converted human mesenchymal stromal cells provide cytoprotective effects on neural stem cells and inhibit their glial differentiation. Cytotherapy 12:491-504

74. Han K, Kim E (2008) Synaptic adhesion molecules and PSD-95. Prog Neurobiol 84:263-283

75. Hanna J, Saha K, Pando B, van Zon J, Lengner CJ, Creyghton MP, van Oudenaarden A, Jaenisch $R$ (2009) Direct cell reprogramming is a stochastic process amenable to acceleration. Nature 462:595-601

76. Hanna J, Cheng AW, Saha K, Kim J, Lengner CJ, Soldner F, Cassady JP, Muffat J, Carey BW, Jaenisch R (2010) Human embryonic stem cells with biological and epigenetic characteristics similar to those of mouse ESCs. Proc Natl Acad Sci U S A 107:9222-9227

77. Harris WA (2003) Specifying motor neurons: up and down and back to front. Nat Neurosci 6:1247-1249 
78. Higuchi A, Ling QD, Ko YA, Chang Y, Umezawa A (2011) Biomaterials for the feeder-free culture of human embryonic stem cells and induced pluripotent stem cells. Chem Rev 111:3021-3035

79. Hochedlinger K, Jaenisch R (2006) Nuclear reprogramming and pluripotency. Nature 441:1061-1067

80. Hochedlinger K, Plath K (2009) Epigenetic reprogramming and induced pluripotency. Development 136:509-523

81. Hu BY, Du ZW, Zhang SC (2009) Differentiation of human oligodendrocytes from pluripotent stem cells. Nat Protoc 4:1614-1622

82. Hu BY, Zhang SC (2009) Differentiation of spinal motor neurons from pluripotent human stem cells. Nat Protoc 4:1295-1304

83. Hu BY, Weick JP, Yu J, Ma LX, Zhang XQ, Thomson JA, Zhang SC (2010) Neural differentiation of human induced pluripotent stem cells follows developmental principles but with variable potency. Proc Natl Acad Sci U S A 107:4335-4340

84. Ince PG, Tomkins J, Slade JY, Thatcher NM, Shaw PJ (1998) Amyotrophic lateral sclerosis associated with genetic abnormalities in the gene encoding $\mathrm{Cu} / \mathrm{Zn}$ superoxide dismutase: molecular pathology of five new cases, and comparison with previous reports and 73 sporadic cases of ALS. J Neuropathol Exp Neurol 57:895-904

85. Jackson M, AI-Chalabi A, Enayat ZE, Chioza B, Leigh PN, Morrison KE (1997) Copper/zinc superoxide dismutase 1 and sporadic amyotrophic lateral sclerosis: analysis of 155 cases and identification of a novel insertion mutation. Ann Neurol 42:803-807

86. James D, Levine AJ, Besser D, Hemmati-Brivanlou A (2005) TGFbeta/activin/nodal signaling is necessary for the maintenance of pluripotency in human embryonic stem cells. Development 132:1273-1282

87. Jessell TM (2000) Neuronal specification in the spinal cord: inductive signals and transcriptional codes. Nat Rev Genet 1:20-29

88. Johnson MA, Weick JP, Pearce RA, Zhang SC (2007) Functional neural development from human embryonic stem cells: accelerated synaptic activity via astrocyte coculture. J Neurosci 27:3069-3077

89. Jucker $M(2010)$ The benefits and limitations of animal models for translational research in neurodegenerative diseases. Nat Med 16:12101214

90. Kang HB, Kim JS, Kwon HJ, Nam KH, Youn HS, Sok DE, Lee Y (2005) Basic fibroblast growth factor activates ERK and induces c-fos in human embryonic stem cell line MizhES1. Stem Cells Dev 14:395-401

91. Kattman SJ, Witty AD, Gagliardi M, Dubois NC, Niapour M, Hotta A, Ellis J, Keller G (2011) Stage-specific optimization of activin/nodal and BMP signaling promotes cardiac differentiation of mouse and human pluripotent stem cell lines. Cell Stem Cell 8:228-240

92. Kim JB, Zaehres H, Wu G, Gentile L, Ko K, Sebastiano V, Arauzo-Bravo MJ, Ruau D, Han DW, Zenke M, Scholer HR (2008) Pluripotent stem cells induced from adult neural stem cells by reprogramming with two factors. Nature 454:646-650

93. Kim JE, O'Sullivan ML, Sanchez CA, Hwang M, Israel MA, Brennand K, Deerinck TJ, Goldstein LS, Gage FH, Ellisman MH, Ghosh A (2011) Investigating synapse formation and function using human pluripotent stem cell-derived neurons. Proc Natl Acad Sci U S A 108:3005-3010

94. Kirsch J (2006) Glycinergic transmission. Cell Tissue Res 326:535-540 
95. Kleger A, Seufferlein T, Malan D, Tischendorf M, Storch A, Wolheim A, Latz S, Protze S, Porzner M, Proepper C, Brunner C, Katz SF, Varma Pusapati G, Bullinger L, Franz WM, Koehntop R, Giehl K, Spyrantis A, Wittekindt O, Lin Q, Zenke M, Fleischmann BK, Wartenberg M, Wobus AM, Boeckers TM, Liebau S (2010) Modulation of calcium-activated potassium channels induces cardiogenesis of pluripotent stem cells and enrichment of pacemaker-like cells. Circulation 122:1823-1836

96. Kleinsmith LJ, Pierce GB, Jr. (1964) Multipotentiality Of Single Embryonal Carcinoma Cells. Cancer Res 24:1544-1551

97. Koch P, Opitz T, Steinbeck JA, Ladewig J, Brustle O (2009) A rosette-type, self-renewing human ES cell-derived neural stem cell with potential for in vitro instruction and synaptic integration. Proc Natl Acad Sci U S A 106:3225-3230

98. Kukekov VG, Laywell ED, Suslov O, Davies K, Scheffler B, Thomas LB, O'Brien TF, Kusakabe M, Steindler DA (1999) Multipotent stem/progenitor cells with similar properties arise from two neurogenic regions of adult human brain. Exp Neurol 156:333-344

99. Kwiatkowski TJ, Jr., Bosco DA, Leclerc AL, Tamrazian E, Vanderburg CR, Russ C, Davis A, Gilchrist J, Kasarskis EJ, Munsat T, Valdmanis P, Rouleau GA, Hosler BA, Cortelli P, de Jong PJ, Yoshinaga Y, Haines JL, Pericak-Vance MA, Yan J, Ticozzi N, Siddique T, McKenna-Yasek D, Sapp PC, Horvitz HR, Landers JE, Brown RH, Jr. (2009) Mutations in the FUS/TLS gene on chromosome 16 cause familial amyotrophic lateral sclerosis. Science 323:1205-1208

100. Lemmens R, Van Hoecke A, Hersmus N, Geelen V, D'Hollander I, Thijs V, Van Den Bosch L, Carmeliet P, Robberecht W (2007) Overexpression of mutant superoxide dismutase 1 causes a motor axonopathy in the zebrafish. Hum Mol Genet 16:2359-2365

101. Lengner CJ (2010) iPS cell technology in regenerative medicine. Ann N Y Acad Sci 1192:38-44

102. Lewitzky M, Yamanaka S (2007) Reprogramming somatic cells towards pluripotency by defined factors. Curr Opin Biotechnol 18:467-473

103. Li J, Wang G, Wang C, Zhao Y, Zhang H, Tan Z, Song Z, Ding M, Deng H (2007) MEK/ERK signaling contributes to the maintenance of human embryonic stem cell self-renewal. Differentiation 75:299-307

104. Li P, Tong C, Mehrian-Shai R, Jia L, Wu N, Yan Y, Maxson RE, Schulze EN, Song H, Hsieh CL, Pera MF, Ying QL (2008) Germline competent embryonic stem cells derived from rat blastocysts. Cell 135:1299-1310

105. Li XJ, Du ZW, Zarnowska ED, Pankratz M, Hansen LO, Pearce RA, Zhang SC (2005) Specification of motoneurons from human embryonic stem cells. Nat Biotechnol 23:215-221

106. Li XJ, Hu BY, Jones SA, Zhang YS, Lavaute T, Du ZW, Zhang SC (2008) Directed differentiation of ventral spinal progenitors and motor neurons from human embryonic stem cells by small molecules. Stem Cells 26:886-893

107. Liebau S, Vaida B, Storch A, Boeckers TM (2007) Maturation of synaptic contacts in differentiating neural stem cells. Stem Cells 25:1720-1729

108. Linta L, Stockmann M, Kleinhans KN, Bockers A, Storch A, Zaehres H, Lin Q, Barbi G, Bockers TM, Kleger A, Liebau S (2012) Rat Embryonic Fibroblasts Improve Reprogramming of Human Keratinocytes into Induced Pluripotent Stem Cells. Stem Cells Dev 
109. Litingtung $Y$, Chiang $C$ (2000) Control of Shh activity and signaling in the neural tube. Dev Dyn 219:143-154

110. Liu JP, Laufer E, Jessell TM (2001) Assigning the positional identity of spinal motor neurons: rostrocaudal patterning of Hox-c expression by FGFs, Gdf11, and retinoids. Neuron 32:997-1012

111. Liu SP, Fu RH, Huang YC, Chen SY, Chien YJ, Hsu CY, Tsai CH, Shyu WC, Lin SZ Induced pluripotent stem (iPS) cell research overview. Cell Transplant 20:15-19

112. Liu SP, Fu RH, Huang YC, Chen SY, Chien YJ, Hsu CY, Tsai CH, Shyu WC, Lin SZ (2011) Induced pluripotent stem (iPS) cell research overview. Cell Transplant 20:15-19

113. Liu SV (2008) iPS cells: a more critical review. Stem Cells Dev 17:391-397

114. Liu Y, Zhang SC (2010) Human stem cells as a model of motoneuron development and diseases. Ann N Y Acad Sci 1198:192-200

115. Lo B, Parham L (2009) Ethical issues in stem cell research. Endocr Rev 30:204-213

116. Loh YH, Wu Q, Chew JL, Vega VB, Zhang W, Chen X, Bourque G, George J, Leong B, Liu J, Wong KY, Sung KW, Lee CW, Zhao XD, Chiu KP, Lipovich L, Kuznetsov VA, Robson P, Stanton LW, Wei CL, Ruan Y, Lim B, $\mathrm{Ng} \mathrm{HH}(2006)$ The Oct4 and Nanog transcription network regulates pluripotency in mouse embryonic stem cells. Nat Genet 38:431-440

117. Loh YH, Agarwal S, Park IH, Urbach A, Huo H, Heffner GC, Kim K, Miller JD, Ng K, Daley GQ (2009) Generation of induced pluripotent stem cells from human blood. Blood 113:5476-5479

118. Ludolph AG, Udvardi PT, Schaz U, Henes C, Adolph O, Weigt HU, Fegert JM, Boeckers TM, Fohr KJ (2010) Atomoxetine acts as an NMDA receptor blocker in clinically relevant concentrations. Br J Pharmacol 160:283-291

119. Ludwig TE, Bergendahl V, Levenstein ME, Yu J, Probasco MD, Thomson JA (2006) Feeder-independent culture of human embryonic stem cells. Nat Methods 3:637-646

120. Ma Y, Peng S, Dhawan V, Eidelberg D (2011) Dopamine cell transplantation in Parkinson's disease: challenge and perspective. Br Med Bull 100:173-189

121. Ma YC, Song MR, Park JP, Henry Ho HY, Hu L, Kurtev MV, Zieg J, Ma Q, Pfaff SL, Greenberg ME (2008) Regulation of motor neuron specification by phosphorylation of neurogenin 2. Neuron 58:65-77

122. Maden M (2007) Retinoic acid in the development, regeneration and maintenance of the nervous system. Nat Rev Neurosci 8:755-765

123. Magrane J, Manfredi G (2009) Mitochondrial function, morphology, and axonal transport in amyotrophic lateral sclerosis. Antioxid Redox Signal 11:1615-1626

124. Magrane J, Sahawneh MA, Przedborski S, Estevez AG, Manfredi G (2012) Mitochondrial dynamics and bioenergetic dysfunction is associated with synaptic alterations in mutant SOD1 motor neurons. J Neurosci 32:229-242

125. Maherali N, Hochedlinger K (2008) Guidelines and techniques for the generation of induced pluripotent stem cells. Cell Stem Cell 3:595-605

126. Maherali N, Hochedlinger K (2009) Tgfbeta signal inhibition cooperates in the induction of iPSCs and replaces Sox2 and cMyc. Curr Biol 19:17181723

127. Mamidi MK, Pal R, Mori NA, Arumugam G, Thrichelvam ST, Noor PJ, Abdullah HM, Gupta PK, Das AK, Zakaria Z, Bhonde R (2011) Co-culture of 
mesenchymal-like stromal cells derived from human foreskin permits long term propagation and differentiation of human embryonic stem cells. J Cell Biochem 112:1353-1363

128. Marchetto MC, Muotri AR, Mu Y, Smith AM, Cezar GG, Gage FH (2008) Non-cell-autonomous effect of human SOD1 G37R astrocytes on motor neurons derived from human embryonic stem cells. Cell Stem Cell 3:649657

129. Martinou JC, Le Van Thai A, Cassar G, Roubinet F, Weber MJ (1989) Characterization of two factors enhancing choline acetyltransferase activity in cultures of purified rat motoneurons. J Neurosci 9:3645-3656

130. Mason I (2007) Initiation to end point: the multiple roles of fibroblast growth factors in neural development. Nat Rev Neurosci 8:583-596

131. Mathews CH, Detmer K, Boncinelli E, Lawrence HJ, Largman C (1991) Erythroid-restricted expression of homeobox genes of the human $\mathrm{HOX} 2$ locus. Blood 78:2248-2252

132. Miller RA, Ruddle FH (1976) Pluripotent teratocarcinoma-thymus somatic cell hybrids. Cell 9:45-55

133. Mintz B, Illmensee K (1975) Normal genetically mosaic mice produced from malignant teratocarcinoma cells. Proc Natl Acad Sci U S A 72:3585-3589

134. Morizane A, Doi D, Kikuchi T, Nishimura K, Takahashi J (2011) Smallmolecule inhibitors of bone morphogenic protein and activin/nodal signals promote highly efficient neural induction from human pluripotent stem cells. $\mathrm{J}$ Neurosci Res

135. Muller M, Stockmann M, Malan D, Wolheim A, Tischendorf M, Linta L, Katz SF, Lin Q, Latz S, Brunner C, Wobus AM, Zenke M, Wartenberg M, Boeckers TM, von Wichert G, Fleischmann BK, Liebau S, Kleger A (2012) $\mathrm{Ca} 2+$ activated $\mathrm{K}$ channels-new tools to induce cardiac commitment from pluripotent stem cells in mice and men. Stem Cell Rev 8:720-740

136. Mumaw JL, Machacek D, Shields JP, Dodla MC, Dhara SK, Stice SL (2010) Neural differentiation of human embryonic stem cells at the ultrastructural level. Microsc Microanal 16:80-90

137. Mummery C, van der Heyden MA, de Boer TP, Passier R, Ward D, van den Brink S, van Rooijen M, van de Stolpe A (2007) Cardiomyocytes from human and mouse embryonic stem cells. Methods Mol Med 140:249-272

138. Munsie MJ, Michalska AE, O'Brien CM, Trounson AO, Pera MF, Mountford PS (2000) Isolation of pluripotent embryonic stem cells from reprogrammed adult mouse somatic cell nuclei. Curr Biol 10:989-992

139. Murray LM, Talbot K, Gillingwater TH (2010) Review: neuromuscular synaptic vulnerability in motor neurone disease: amyotrophic lateral sclerosis and spinal muscular atrophy. Neuropathol Appl Neurobiol 36:133156

140. Nakagawa M, Koyanagi M, Tanabe K, Takahashi K, Ichisaka T, Aoi T, Okita K, Mochiduki Y, Takizawa N, Yamanaka S (2008) Generation of induced pluripotent stem cells without Myc from mouse and human fibroblasts. Nat Biotechnol 26:101-106

141. Neumann M, Sampathu DM, Kwong LK, Truax AC, Micsenyi MC, Chou TT, Bruce J, Schuck T, Grossman M, Clark CM, McCluskey LF, Miller BL, Masliah E, Mackenzie IR, Feldman H, Feiden W, Kretzschmar HA, Trojanowski JQ, Lee VM (2006) Ubiquitinated TDP-43 in frontotemporal lobar degeneration and amyotrophic lateral sclerosis. Science 314:130-133 
142. O'Connor MD, Kardel MD, Eaves CJ (2011) Functional assays for human embryonic stem cell pluripotency. Methods Mol Biol 690:67-80

143. Okabe S, Forsberg-Nilsson K, Spiro AC, Segal M, McKay RD (1996) Development of neuronal precursor cells and functional postmitotic neurons from embryonic stem cells in vitro. Mech Dev 59:89-102

144. Okada Y, Shimazaki T, Sobue G, Okano H (2004) Retinoic-acidconcentration-dependent acquisition of neural cell identity during in vitro differentiation of mouse embryonic stem cells. Dev Biol 275:124-142

145. Okita K, Nakagawa M, Hyenjong H, Ichisaka T, Yamanaka S (2008) Generation of mouse induced pluripotent stem cells without viral vectors. Science 322:949-953

146. Pankratz MT, Li XJ, Lavaute TM, Lyons EA, Chen X, Zhang SC (2007) Directed neural differentiation of human embryonic stem cells via an obligated primitive anterior stage. Stem Cells 25:1511-1520

147. Park Y, Kim JH, Lee SJ, Choi IY, Park SJ, Lee SR, Sung HJ, Yoo YD, Geum DH, Choi CW, Kim SH, Kim BS (2011) Human Feeder Cells Can Support the Undifferentiated Growth of Human and Mouse Embryonic Stem Cells Using Their Own Basic Fibroblast Growth Factors. Stem Cells Dev

148. Patel M, Yang S (2010) Advances in reprogramming somatic cells to induced pluripotent stem cells. Stem Cell Rev 6:367-380

149. Pera MF, Cooper S, Mills J, Parrington JM (1989) Isolation and characterization of a multipotent clone of human embryonal carcinoma cells. Differentiation 42:10-23

150. Pera MF, Tam PP (2010) Extrinsic regulation of pluripotent stem cells. Nature 465:713-720

151. Placzek M (1995) The role of the notochord and floor plate in inductive interactions. Curr Opin Genet Dev 5:499-506

152. Polo JM, Liu S, Figueroa ME, Kulalert W, Eminli S, Tan KY, Apostolou E, Stadtfeld M, Li Y, Shioda T, Natesan S, Wagers AJ, Melnick A, Evans T, Hochedlinger K (2010) Cell type of origin influences the molecular and functional properties of mouse induced pluripotent stem cells. Nat Biotechnol 28:848-855

153. Puls I, Jonnakuty C, LaMonte BH, Holzbaur EL, Tokito M, Mann E, Floeter MK, Bidus K, Drayna D, Oh SJ, Brown RH, Jr., Ludlow CL, Fischbeck KH (2003) Mutant dynactin in motor neuron disease. Nat Genet 33:455-456

154. Ramirez JM, Bai Q, Dijon-Grinand M, Assou S, Gerbal-Chaloin S, Hamamah S, De Vos J (2010) Human pluripotent stem cells: from biology to cell therapy. World J Stem Cells 2:24-33

155. Ramirez JM, Gerbal-Chaloin S, Milhavet O, Qiang B, Becker F, Assou S, Lemaitre JM, Hamamah S, De Vos J (2011) Brief report: benchmarking human pluripotent stem cell markers during differentiation into the three germ layers unveils a striking heterogeneity: all markers are not equal. Stem Cells 29:1469-1474

156. Raya A, Rodriguez-Piza I, Guenechea G, Vassena R, Navarro S, Barrero MJ, Consiglio A, Castella M, Rio P, Sleep E, Gonzalez F, Tiscornia G, Garreta E, Aasen T, Veiga A, Verma IM, Surralles J, Bueren J, Izpisua Belmonte JC (2009) Disease-corrected haematopoietic progenitors from Fanconi anaemia induced pluripotent stem cells. Nature 460:53-59

157. Reubinoff BE, Pera MF, Fong CY, Trounson A, Bongso A (2000) Embryonic stem cell lines from human blastocysts: somatic differentiation in vitro. Nat Biotechnol 18:399-404 
158. Reubinoff BE, Itsykson P, Turetsky T, Pera MF, Reinhartz E, Itzik A, BenHur T (2001) Neural progenitors from human embryonic stem cells. Nat Biotechnol 19:1134-1140

159. Reynolds BA, Weiss S (1992) Generation of neurons and astrocytes from isolated cells of the adult mammalian central nervous system. Science 255:1707-1710

160. Rosen DR, Siddique T, Patterson D, Figlewicz DA, Sapp P, Hentati A, Donaldson D, Goto J, O'Regan JP, Deng HX, et al. (1993) Mutations in $\mathrm{Cu} / \mathrm{Zn}$ superoxide dismutase gene are associated with familial amyotrophic lateral sclerosis. Nature 362:59-62

161. Sala C, Piech V, Wilson NR, Passafaro M, Liu G, Sheng M (2001) Regulation of dendritic spine morphology and synaptic function by Shank and Homer. Neuron 31:115-130

162. Sampedro MN, Bussineau CM, Cotman CW (1981) Postsynaptic density antigens: preparation and characterization of an antiserum against postsynaptic densities. J Cell Biol 90:675-686

163. Sanchez-Ponce D, Tapia M, Munoz A, Garrido JJ (2008) New role of IKK alpha/beta phosphorylated I kappa B alpha in axon outgrowth and axon initial segment development. Mol Cell Neurosci 37:832-844

164. Sanes JR, Marshall LM, McMahan UJ (1978) Reinnervation of muscle fiber basal lamina after removal of myofibers. Differentiation of regenerating axons at original synaptic sites. J Cell Biol 78:176-198

165. Sanes JR, Lichtman JW (2001) Induction, assembly, maturation and maintenance of a postsynaptic apparatus. Nat Rev Neurosci 2:791-805

166. Schmeisser MJ, Grabrucker AM, Bockmann J, Boeckers TM (2009) Synaptic cross-talk between $\mathrm{N}$-methyl-D-aspartate receptors and LAPSER1-beta-catenin at excitatory synapses. J Biol Chem 284:2914629157

167. Schulz TC, Palmarini GM, Noggle SA, Weiler DA, Mitalipova MM, Condie BG (2003) Directed neuronal differentiation of human embryonic stem cells. BMC Neurosci 4:27

168. Schulz TC, Noggle SA, Palmarini GM, Weiler DA, Lyons IG, Pensa KA, Meedeniya AC, Davidson BP, Lambert NA, Condie BG (2004)

Differentiation of human embryonic stem cells to dopaminergic neurons in serum-free suspension culture. Stem Cells 22:1218-1238

169. Shamblott MJ, Axelman J, Wang S, Bugg EM, Littlefield JW, Donovan PJ, Blumenthal PD, Huggins GR, Gearhart JD (1998) Derivation of pluripotent stem cells from cultured human primordial germ cells. Proc Natl Acad Sci U S A 95:13726-13731

170. Shamblott MJ, Axelman J, Littlefield JW, Blumenthal PD, Huggins GR, Cui Y, Cheng L, Gearhart JD (2001) Human embryonic germ cell derivatives express a broad range of developmentally distinct markers and proliferate extensively in vitro. Proc Natl Acad Sci U S A 98:113-118

171. Shapira M, Zhai RG, Dresbach T, Bresler T, Torres VI, Gundelfinger ED, Ziv NE, Garner CC (2003) Unitary assembly of presynaptic active zones from Piccolo-Bassoon transport vesicles. Neuron 38:237-252

172. Shaw PJ (2005) Molecular and cellular pathways of neurodegeneration in motor neurone disease. J Neurol Neurosurg Psychiatry 76:1046-1057

173. Shi P, Wei Y, Zhang J, Gal J, Zhu H (2010) Mitochondrial dysfunction is a converging point of multiple pathological pathways in amyotrophic lateral sclerosis. J Alzheimers Dis 20 Suppl 2:S311-324 
174. Shin S, Mitalipova M, Noggle S, Tibbitts D, Venable A, Rao R, Stice SL (2006) Long-term proliferation of human embryonic stem cell-derived neuroepithelial cells using defined adherent culture conditions. Stem Cells 24:125-138

175. Sigurjonsson OE, Perreault MC, Egeland T, Glover JC (2005) Adult human hematopoietic stem cells produce neurons efficiently in the regenerating chicken embryo spinal cord. Proc Natl Acad Sci U S A 102:5227-5232

176. Silva SS, Rowntree RK, Mekhoubad S, Lee JT (2008) X-chromosome inactivation and epigenetic fluidity in human embryonic stem cells. Proc Natl Acad Sci U S A 105:4820-4825

177. Smith AG, Heath JK, Donaldson DD, Wong GG, Moreau J, Stahl M, Rogers D (1988) Inhibition of pluripotential embryonic stem cell differentiation by purified polypeptides. Nature 336:688-690

178. Somers A, Jean JC, Sommer CA, Omari A, Ford CC, Mills JA, Ying L, Sommer AG, Jean JM, Smith BW, Lafyatis R, Demierre MF, Weiss DJ, French DL, Gadue P, Murphy GJ, Mostoslavsky G, Kotton DN (2010) Generation of transgene-free lung disease-specific human induced pluripotent stem cells using a single excisable lentiviral stem cell cassette. Stem Cells 28:1728-1740

179. Sommer CA, Stadtfeld M, Murphy GJ, Hochedlinger K, Kotton DN, Mostoslavsky G (2009) Induced pluripotent stem cell generation using a single lentiviral stem cell cassette. Stem Cells 27:543-549

180. Sommer CA, Sommer AG, Longmire TA, Christodoulou C, Thomas DD, Gostissa M, Alt FW, Murphy GJ, Kotton DN, Mostoslavsky G (2010) Excision of reprogramming transgenes improves the differentiation potential of iPS cells generated with a single excisable vector. Stem Cells 28:64-74

181. Song JY, Ichtchenko K, Sudhof TC, Brose N (1999) Neuroligin 1 is a postsynaptic cell-adhesion molecule of excitatory synapses. Proc Natl Acad Sci U S A 96:1100-1105

182. Sonntag KC, Pruszak J, Yoshizaki T, van Arensbergen J, SanchezPernaute R, Isacson O (2007) Enhanced yield of neuroepithelial precursors and midbrain-like dopaminergic neurons from human embryonic stem cells using the bone morphogenic protein antagonist noggin. Stem Cells 25:411418

183. Sreedharan J, Blair IP, Tripathi VB, Hu X, Vance C, Rogelj B, Ackerley S, Durnall JC, Williams KL, Buratti E, Baralle F, de Belleroche J, Mitchell JD, Leigh PN, Al-Chalabi A, Miller CC, Nicholson G, Shaw CE (2008) TDP-43 mutations in familial and sporadic amyotrophic lateral sclerosis. Science 319:1668-1672

184. Stevens LC (1967) Origin of testicular teratomas from primordial germ cells in mice. J Natl Cancer Inst 38:549-552

185. Stockmann M, Linta L, Fohr KJ, Boeckers A, Ludolph AC, Kuh GF, Udvardi PT, Proepper C, Storch A, Kleger A, Liebau S, Boeckers TM (2011)

Developmental and Functional Nature of Human iPSC Derived Motoneurons. Stem Cell Rev

186. Stockmann M, Meyer-Ohlendorf M, Achberger K, Putz S, Demestre M, Yin $\mathrm{H}$, Hendrich C, Linta L, Heinrich J, Brunner C, Proepper C, Kuh GF, Baumann B, Langer T, Schwalenstocker B, Braunstein KE, von Arnim C, Schneuwly S, Meyer T, Wong PC, Boeckers TM, Ludolph AC, Liebau S (2012) The dynactin p150 subunit: cell biology studies of sequence 
changes found in ALS/MND and Parkinsonian Syndromes. J Neural Transm

187. Stojkovic M, Lako M, Strachan T, Murdoch A (2004) Derivation, growth and applications of human embryonic stem cells. Reproduction 128:259-267

188. Storch A, Paul G, Csete M, Boehm BO, Carvey PM, Kupsch A, Schwarz J (2001) Long-term proliferation and dopaminergic differentiation of human mesencephalic neural precursor cells. Exp Neurol 170:317-325

189. Storch A, Lester HA, Boehm BO, Schwarz J (2003) Functional characterization of dopaminergic neurons derived from rodent mesencephalic progenitor cells. J Chem Neuroanat 26:133-142

190. Tada M, Tada T, Lefebvre L, Barton SC, Surani MA (1997) Embryonic germ cells induce epigenetic reprogramming of somatic nucleus in hybrid cells. Embo J 16:6510-6520

191. Tada M, Morizane A, Kimura H, Kawasaki H, Ainscough JF, Sasai Y, Nakatsuji N, Tada T (2003) Pluripotency of reprogrammed somatic genomes in embryonic stem hybrid cells. Dev Dyn 227:504-510

192. Takahashi K, Yamanaka S (2006) Induction of pluripotent stem cells from mouse embryonic and adult fibroblast cultures by defined factors. Cell 126:663-676

193. Tarasenko YI, Yu Y, Jordan PM, Bottenstein J, Wu P (2004) Effect of growth factors on proliferation and phenotypic differentiation of human fetal neural stem cells. J Neurosci Res 78:625-636

194. Tatariunas AB (1991) [Accumulation of lipofuscin granules in acute myocardial infarct as a model of cell aging]. Biull Eksp Biol Med 111:443446

195. Thomson JA, Itskovitz-Eldor J, Shapiro SS, Waknitz MA, Swiergiel JJ, Marshall VS, Jones JM (1998) Embryonic stem cell lines derived from human blastocysts. Science 282:1145-1147

196. Thomson JA, Marshall VS (1998) Primate embryonic stem cells. Curr Top Dev Biol 38:133-165

197. Thomson JA, Odorico JS (2000) Human embryonic stem cell and embryonic germ cell lines. Trends Biotechnol 18:53-57

198. Thomson SR, Wishart TM, Patani R, Chandran S, Gillingwater TH (2012) Using induced pluripotent stem cells (iPSC) to model human neuromuscular connectivity: promise or reality? J Anat

199. tom Dieck S, Sanmarti-Vila L, Langnaese K, Richter K, Kindler S, Soyke A, Wex H, Smalla KH, Kampf U, Franzer JT, Stumm M, Garner CC, Gundelfinger ED (1998) Bassoon, a novel zinc-finger CAG/glutaminerepeat protein selectively localized at the active zone of presynaptic nerve terminals. J Cell Biol 142:499-509

200. Tu JC, Xiao B, Naisbitt S, Yuan JP, Petralia RS, Brakeman P, Doan A, Aakalu VK, Lanahan AA, Sheng M, Worley PF (1999) Coupling of $\mathrm{mGluR} /$ Homer and PSD-95 complexes by the Shank family of postsynaptic density proteins. Neuron 23:583-592

201. Ueda S, Kawamata M, Teratani T, Shimizu T, Tamai Y, Ogawa H, Hayashi $\mathrm{K}$, Tsuda H, Ochiya $\mathrm{T}$ (2008) Establishment of rat embryonic stem cells and making of chimera rats. PLoS One 3:e2800

202. Vallier L, Alexander M, Pedersen RA (2005) Activin/Nodal and FGF pathways cooperate to maintain pluripotency of human embryonic stem cells. J Cell Sci 118:4495-4509 
203. Vance C, Rogelj B, Hortobagyi T, De Vos KJ, Nishimura AL, Sreedharan J, Hu X, Smith B, Ruddy D, Wright P, Ganesalingam J, Williams KL, Tripathi V, Al-Saraj S, Al-Chalabi A, Leigh PN, Blair IP, Nicholson G, de Belleroche J, Gallo JM, Miller CC, Shaw CE (2009) Mutations in FUS, an RNA processing protein, cause familial amyotrophic lateral sclerosis type 6 . Science 323:1208-1211

204. Voelker CC, Garin N, Taylor JS, Gahwiler BH, Hornung JP, Molnar Z (2004) Selective neurofilament (SMI-32, FNP-7 and N200) expression in subpopulations of layer $\mathrm{V}$ pyramidal neurons in vivo and in vitro. Cereb Cortex 14:1276-1286

205. Wakayama S, Jakt ML, Suzuki M, Araki R, Hikichi T, Kishigami S, Ohta H, Van Thuan N, Mizutani E, Sakaide Y, Senda S, Tanaka S, Okada M, Miyake M, Abe M, Nishikawa S, Shiota K, Wakayama T (2006) Equivalency of nuclear transfer-derived embryonic stem cells to those derived from fertilized mouse blastocysts. Stem Cells 24:2023-2033

206. Walsh RM, Hochedlinger K (2010) Modeling Rett syndrome with stem cells. Cell 143:499-500

207. Warren L, Manos PD, Ahfeldt T, Loh YH, Li H, Lau F, Ebina W, Mandal PK, Smith ZD, Meissner A, Daley GQ, Brack AS, Collins JJ, Cowan C, Schlaeger TM, Rossi DJ (2010) Highly efficient reprogramming to pluripotency and directed differentiation of human cells with synthetic modified mRNA. Cell Stem Cell 7:618-630

208. Watanabe K, Kamiya D, Nishiyama A, Katayama T, Nozaki S, Kawasaki H, Watanabe Y, Mizuseki K, Sasai Y (2005) Directed differentiation of telencephalic precursors from embryonic stem cells. Nat Neurosci 8:288296

209. Weihe E, Tao-Cheng JH, Schafer MK, Erickson JD, Eiden LE (1996) Visualization of the vesicular acetylcholine transporter in cholinergic nerve terminals and its targeting to a specific population of small synaptic vesicles. Proc Natl Acad Sci U S A 93:3547-3552

210. Welles JK, Wrye HK (1991) The maternal erotic countertransference. Int J Psychoanal 72 (Pt 1):93-106

211. Wernig M, Meissner A, Foreman R, Brambrink T, Ku M, Hochedlinger K, Bernstein BE, Jaenisch $\mathrm{R}$ (2007) In vitro reprogramming of fibroblasts into a pluripotent ES-cell-like state. Nature 448:318-324

212. Wichterle H, Lieberam I, Porter JA, Jessell TM (2002) Directed differentiation of embryonic stem cells into motor neurons. Cell 110:385-397

213. Willems E, Spiering S, Davidovics H, Lanier M, Xia Z, Dawson M, Cashman J, Mercola M (2011) Small-molecule inhibitors of the wnt pathway potently promote cardiomyocytes from human embryonic stem cell-derived mesoderm. Circ Res 109:360-364

214. Wobus AM, Boheler KR (2005) Embryonic stem cells: prospects for developmental biology and cell therapy. Physiol Rev 85:635-678

215. Wu H, Xiong WC, Mei L (2012) To build a synapse: signaling pathways in neuromuscular junction assembly. Development 137:1017-1033

216. Xiao L, Yuan X, Sharkis SJ (2006) Activin A maintains self-renewal and regulates fibroblast growth factor, Wnt, and bone morphogenic protein pathways in human embryonic stem cells. Stem Cells 24:1476-1486

217. Xu C, Inokuma MS, Denham J, Golds K, Kundu P, Gold JD, Carpenter MK (2001) Feeder-free growth of undifferentiated human embryonic stem cells. Nat Biotechnol 19:971-974 
218. Xu RH, Chen X, Li DS, Li R, Addicks GC, Glennon C, Zwaka TP, Thomson JA (2002) BMP4 initiates human embryonic stem cell differentiation to trophoblast. Nat Biotechnol 20:1261-1264

219. Xu RH, Peck RM, Li DS, Feng X, Ludwig T, Thomson JA (2005) Basic FGF and suppression of BMP signaling sustain undifferentiated proliferation of human ES cells. Nat Methods 2:185-190

220. Yang L, Soonpaa MH, Adler ED, Roepke TK, Kattman SJ, Kennedy M, Henckaerts E, Bonham K, Abbott GW, Linden RM, Field LJ, Keller GM (2008) Human cardiovascular progenitor cells develop from a KDR+ embryonic-stem-cell-derived population. Nature 453:524-528

221. Ye Z, Cheng $L$ (2010) Potential of human induced pluripotent stem cells derived from blood and other postnatal cell types. Regen Med 5:521-530

222. Yoshida K, Chambers I, Nichols J, Smith A, Saito M, Yasukawa K, Shoyab M, Taga T, Kishimoto T (1994) Maintenance of the pluripotential phenotype of embryonic stem cells through direct activation of gp130 signalling pathways. Mech Dev 45:163-171

223. Yu J, Vodyanik MA, Smuga-Otto K, Antosiewicz-Bourget J, Frane JL, Tian S, Nie J, Jonsdottir GA, Ruotti V, Stewart R, Slukvin, II, Thomson JA (2007) Induced pluripotent stem cell lines derived from human somatic cells. Science 318:1917-1920

224. Yu J, Thomson JA (2008) Pluripotent stem cell lines. Genes Dev 22:19871997

225. Zhai R, Olias G, Chung WJ, Lester RA, tom Dieck S, Langnaese K, Kreutz MR, Kindler S, Gundelfinger ED, Garner CC (2000) Temporal appearance of the presynaptic cytomatrix protein bassoon during synaptogenesis. Mol Cell Neurosci 15:417-428

226. Zhang H, Wu CY, Wang W, Harrington MA (2011) Interneuronal synapses formed by motor neurons appear to be glutamatergic. Neuroreport 22:809813

227. Zhang K, Cai Z, Li Y, Shu J, Pan L, Wan F, Li H, Huang X, He C, Liu Y, Cui X, Xu Y, Gao Y, Wu L, Cao S, Li L (2011) Utilization of human amniotic mesenchymal cells as feeder layers to sustain propagation of human embryonic stem cells in the undifferentiated state. Cell Reprogram 13:281288

228. Zhang SC, Wernig M, Duncan ID, Brustle O, Thomson JA (2001) In vitro differentiation of transplantable neural precursors from human embryonic stem cells. Nat Biotechnol 19:1129-1133

229. Zhang SC (2006) Neural subtype specification from embryonic stem cells. Brain Pathol 16:132-142

230. Zhang XQ, Zhang SC (2010) Differentiation of neural precursors and dopaminergic neurons from human embryonic stem cells. Methods Mol Biol 584:355-366

231. Zumla A, Williams W, Mudd D, Locniskar M, Behrens R, Isenberg D, McAdam KP (1991) Expression of a common idiotype PR4 in the sera of patients with leprosy. Clin Exp Immunol 84:522-526

232. Zwaka TP, Thomson JA (2003) Homologous recombination in human embryonic stem cells. Nat Biotechnol 21:319-321 


\section{Figures and Tables}

Fig. 1 Extrinsic regulation of Pluripotency

Fig. 2 Stem Cell Development

Fig. 3 Development of the Spinal Cord and postmitotic Motoneurons

Fig. 4 Generation of human induced pluripotent Stem Cells from Keratinocytes

Fig. 5 Differentiation of Keratinocytes derived hiPSCs into Motoneurons

Fig. 6 Human iPSCs derived Motoneurons express all Signs of functional Neurons

Fig. 7 Time Course of the Development of hiPSCs derived Motoneurons

Fig. 8 Development of a mature Axon

Fig. 9 Development of the Presynapse

Fig. 10 Development of the Postsynapse

Fig. 11 Characterization of the developing Synapses

Fig. 12 Quantification of the developing Synapses

Fig. 13 Co-culture of hiPSCs derived Motoneurons and Myotubes

Fig. 14 Functionality of neuromuscular Junctions

Fig. 15 Time Course of the Development of rat Motoneurons

Fig. 16 Developing Synapses in rat Motoneuron Cultures

Fig. 17 Quantification of Synapses in rat Motoneuron Cultures

Fig. 18 Time Frame of neuronal and synaptic Maturation in hiPSCs derived Motoneurons

Table 1 Primary Antibodies

Table 2 Secondary Antibodies

Table 3 PCR Primers

Table 4 QuantiTec Primers 


\section{Acknowledgements}

I would especially like to thank my supervisor Stefan Liebau. Stefan, thanks a lot for all your advices and support during my thesis. Thank you for giving me the possibility of trying out my own ideas and special thanks for your support during the paper-submission. Spending a research year in your working group was a great experience and this time was definitively the most interesting and enjoyable time during my medical study in Ulm.

Special thanks go to Prof. Böckers and Prof. Ludolph. I would like to thank them for their support during my thesis and for giving me the chance to take part in different meetings. Furthermore I would like to thank them supporting me in combining my medical study with research work.

Furthermore, I would like to thank Leonhard Linta for teaching me different methods and his patience when teaching me how to calculate different molecular weights. Leo, I really enjoyed working with you! Additionally, I would like to thank Sabine Seltenheim for her support in the lab and for her encouraging words in many situations. I also would like to thank Jasmin Haderspeck and Kevin Achberger. I really enjoyed working with you and planning new experiments. Because of you both I can still take part in some research projects although I have to continue my medical study - thanks a lot.

I would especially like to thank Alexander Kleger for all his support and advices. Alex, thanks a lot for giving me the opportunity to show that EBIO is also working in human cells. I really enjoyed this project.

I would like to thank Karl Föhr for performing the electrophysiological measurements, which have been an important part of my publication. I also want to thank him for the correction of my thesis.

I would like to thank Christian Pröppers and Patrick Udvardi for all support and for a great time. 


\section{Acknowledgements}

Special thanks go to Clair Weidgang. Clair, I thank you a lot for your help during the laboratory work and during the exam periods.

Furthermore, I would especially like to thank my boyfriend Manuel Klövekorn for his understanding and his support in every situation.

I would like to express my deepest gratitude to my parents, Barbara and Rudolf Stockmann, who have always supported me in all what I have done and pursued.

And finally, I would like to thank the whole team of the Institute for Anatomy and Cell Biology and the Promotionsprogramm Experimentelle Medizin of the University of Ulm. 
Curriculum vitae

\section{Curriculum vitae}

Lebenslauf aus Gründen des Datenschutzes entfernt. 